\title{
Bioavailability and metabolism of food bioactives and their health effects: a review
}

\author{
Fereidoon Shahidi*, Vegneshwaran Vasudevan Ramakrishnan and Won Young Oh
}

\author{
Department of Biochemistry, Memorial University of Newfoundland, St. John's, NL, A1B 3X9, Canada \\ ${ }^{*}$ Corresponding author: Fereidoon Shahidi, Department of Biochemistry, Memorial University of Newfoundland, St. John's, NL, A1B \\ 3X9, Canada. E-mail: fshahidi@mun.ca \\ DOI: $10.31665 /$ JFB.2019.8204 \\ Received: December 25, 2019; Revised received \& accepted: December 30, 2019 \\ Citation: Shahidi, F., Vasudevan Ramakrishnan, V., and Oh, W.Y. (2019). Bioavailability and metabolism of food bioactives and their health \\ effects: a review. J. Food Bioact. 8: 6-41.
}

\begin{abstract}
Numerous studies have demonstrated the availability of high-quality bioactive compounds in food along with their determination and quantification techniques. Many of these identified compounds have been claimed to possess health benefits such as anti-inflammatory, anti-cancer, antioxidant, anti-allergic, anti-fungal, antimicrobial and cardioprotective spasmolytic properties. However, mere presence of these compounds does not directly correlate with their potential health effects upon consumption. Since any food consumed does not reach the blood stream in the original form and as they are broken down to various compounds it is mandatory to consider their bioavailability and metabolism that takes place upon absorption. Furthermore, the efficacy of these bioactive compounds depends on various factors including the dosage, food matrix and stability of the compound during metabolism. Various bioavailability studies indicate that the parent bioactive compound is broken into various metabolites via oxidation, dehydroxylation, de-esterification, hydrolysis, carboxylation, $\alpha$ - and $\beta$-oxidation processes inside the human body. Therefore, this leads to an interesting conundrum that whether the proposed health effects are due to the parent bioactive compound or due to the metabolites formed during absorption. Some details in relation to the metabolism and metabolites of food bioactives are presented in this contribution.
\end{abstract}

Keywords: Phenolic compounds; Bioavailability; Absorption; Metabolism; Health effect.

\section{Introduction}

Bioactive compounds are non-nutritive constituents in food with potential health-promoting benefits when ingested. Plant-based food including fruits, vegetables and grains, red meat, poultry and seafood, algae, seaweeds and other forms of aquatic products contain various classes of bioactive compounds (Miyashita and Hosokawa, 2018; Mirfat et al., 2018). These bioactive compounds vary widely in their chemical structures and fucntion and are classified accordingly into major groups such as polyphenols, carotenoids, peptides and amino acids, lipids and fatty acids, polysaccharides, glucosinolates, terpenoids, alkaloids and furocoumarins. Eventhough these compouds are known to pro- vide health effects to humans, their bioavailablity is not always well known. The mechanism of bioavailablity involves various metabolic steps right from the start when the food is ingested via mouth, broken down by the action of grinding, effect of saliva juice, travel to the stomach, effect of gastric juice and enzymes in the stomach, the effect of microbiota in the duodenum, jejunum, ileum and colon. In addition, diffusion across the enterocytes and colonocytes, effect of hepatic metabolism in liver and systemic circulation also play an important role in the absorption of these compounds from food sources. Therefore, the aim of this review is to demonstrate various metabolic pathways of known polyphenols present in foods, bioavailability, and their effect on health and disease. 
Table 1. Classification of phenolic and polyphenolic compounds

\begin{tabular}{|c|c|c|}
\hline Class & Subgroup & Example \\
\hline \multirow[t]{7}{*}{ Phenolic acid } & Benzoic acid & Protocatechuic acid \\
\hline & & Vanillic acid \\
\hline & & Gallic acid \\
\hline & Cinnamic acid & $p$-Coumaric acid \\
\hline & & Caffeic acid \\
\hline & & Chlorogenic acid \\
\hline & & Sinapic acid \\
\hline \multirow[t]{2}{*}{ Stilbene } & & Resveratrol \\
\hline & & Pterostilbene \\
\hline \multirow[t]{17}{*}{ Flavonoid } & Flavonol & Quercetin \\
\hline & & Kaempferol \\
\hline & & Myricetin \\
\hline & Flavones & Apigenin \\
\hline & & Acacetin \\
\hline & & Luteolin \\
\hline & Flavanone & Naringenin \\
\hline & & Hesperetin \\
\hline & Flavanol & Catechin \\
\hline & & Epicatechin \\
\hline & & Epigallocatechin \\
\hline & & Epigallocatechin gallate \\
\hline & Anthocyanidin & Cyanidin \\
\hline & & Malvidin \\
\hline & & Petunidin \\
\hline & Isoflavones & Genistein \\
\hline & & Daidzin \\
\hline \multirow[t]{3}{*}{ Lignan } & & Secoisolariciresinol \\
\hline & & Matairesinol \\
\hline & & Syringaresinol \\
\hline \multirow[t]{4}{*}{ Tannin } & Hydrolyzable & Gallotannin \\
\hline & & Ellagitannin \\
\hline & & Tannic acid \\
\hline & Condensed & Proanthocyanidin \\
\hline
\end{tabular}

\section{Phenolic compounds}

Phenolic compounds are the largest group of phytochemicals that are mainly found in plant-based foods. These have one or more phenolic rings that are classified into different subgroups based on their origin, biological function and chemical structure. The different classes of phenolic compounds are phenolic acids, flavonoids, tannins, stilbenes and lignans are shown in Table 1 (Shahidi and Ambigaipalan, 2015; Bolling et al., 2011; Hardman, 2014; Pietta et al., 2003; Tsao, 2010; Shahidi and Yeo, 2016).

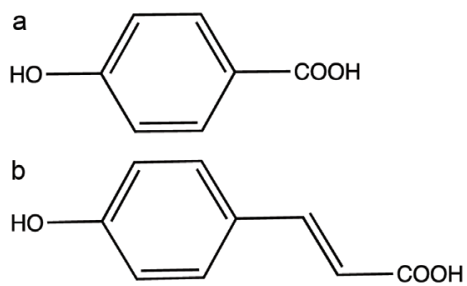

Figure 1. Phenolic acids (a) 4-hydroxybenzoic acid and (b) 4-hydroxycinnamic acid.

\subsection{Phenolic acids}

Phenolic acids are divided into two classes depending on their structures: (a) derivatives of benzoic acid and (b) derivatives of cinnamic acid (Shahidi and Yeo, 2016).

\subsubsection{Hydroxybenzoic acid derivatives (HBA)}

The hydroxybenzoic acid derivatives have a general structure of $\mathrm{C}_{6}-\mathrm{C}_{1}$. Strictly speaking, these are the only phenolic acid derivatives, since hydroxycinnamic acid that will be discussed below are phenylpropanoids (Shahidi and Ambigaipalan, 2015). In some cases, $\mathrm{C}_{6}-\mathrm{C}_{2}$ phenylacetic acid derivatives occur occasionally as minor components in food. Generally, the concentration of HBAs are low in fruits and vegetables except in red fruits, black raddish, onion and potato skin (Lafay and Gil-Izquierdo, 2008). The general structure of hydroxybenzoic acid is shown in Figure 1. Some of the common HBAs include salicylic acid, 4-hydroxybenzoic acid, protocatechuic acid, gallic acid, vanillic acid, isovanillic acid and syringic acid. Cinnamon bark contains protocatechuic acid, salicylic and syringic acids (Muhammad and Dewettinck, 2017). Gallic acid is found in clove buds along with protocatechuic acid, $p$-hydroxybenzoic acid and syringic acid (Tomás-Barberán and Clifford, 2000a). Canadian wheat flours contain vanillic acid and syringic acid (Hatcher and Kruger, 1997; Kim et al., 2018; Yu and Beta, 2015). Similarly, oats, barley, malt, hops contain different forms of hydroxybenzoic acids. Although hydroxybenzoic acids can be found as free acids in some fruits or released after processing, they mainly occur as conjugates. Gallic acid and its dimer such as ellagic acid are esterified with glucose to produce hydrolysable tannins. Also, $p$-hydroxybenzoic acid, vanillic acid, syringic acid and protocatechuic acid are constituents of lignin (Pietta et al., 2003).

\subsubsection{Hydroxycinnamic acid derivatives (HCA)}

The hydroxycinnamic acids (Figure 1) are trans-phenyl-3-propenoic acids with different ring substitutions. Derivatives of cinnamic acids are mainly represented by caffeic, ferulic, sinapic and $p$-coumaric acids. These compounds are often distributed as conjugates mainly as esters of quinic acid. Among hydroxycinnamic acids, caffeic acid is the most common and accounts up to $70 \%$ of total hydroxycinnamic acids in fruits (Carrera et al., 2010; Licciardello et al., 2018). Rosemary extract contains HCA, such as caffeic acid, ferulic acid, and $p$-coumaric acid (Senanayake, 2018). Ferulic acids are present in cereal grains. Since hydroxycinnamic acids occur is most fruits and vegetables, they make significantly larger contribution to total polyphenol intake compared to hydroxybenzoic acids and flavonoids. 
<smiles>c1ccc(C2CCc3ccccc3O2)cc1</smiles>

\section{Flavonoid}<smiles>CCOCCOCCCO</smiles><smiles>CCCCOCCCCCO</smiles><smiles>Oc1cc2ccccc2[o+]c1-c1ccccc1</smiles>

Anthocyanidin<smiles>O=c1c(O)c(-c2ccccc2)oc2ccccc12</smiles>

Flavonol<smiles>CCOCCOCCOCCOCCOCCO</smiles><smiles>OC1Cc2ccccc2OC1c1ccccc1</smiles>

Flavan-3-ol

Figure 2. Basic structures of flavonoids.

\subsection{Flavonoids}

Flavonoids are a group of natural products which are made up of diphenylpropanes (C6-C3-C6) consisting of two aromatic rings linked through three carbons that usually form an oxygenated heterocycle (Alvarez-Parrilla et al., 2018). They are mainly found in fruits, vegetables, grains, bark, roots, stems, and flowers. Basic skeleton of flavonoids (Figure 2) is made up of polyphenolic structure and it is divided into different subgroups depending on the carbon of the $\mathrm{C}$ ring on which $\mathrm{B}$ ring is attached and the degree of unsaturation and oxidation of the $\mathrm{C}$ ring. Flavonoids are composed of 6 subgroups which include flavones, flavonols, isoflavones, flavanones, flavanols and proanthocyanidins, and anthocyanidins and anthocyanins (Panche et al., 2016). Biochemical properties of flavonoids are highly related to their chemical structures (Shahidi and Ambigaipalan, 2015). The nomenclature of all flavonoids with different ring substitutions is shown in Table 2.

\subsubsection{Flavones}

Flavones are one of the most important subgroups of flavonoids due to their biological activities in vitro and in vivo. Flavones (Figure 2) are mostly found in leaves, flowers and fruits as 7-Oglycosides and can also have acetyl or malonyl moieties (Panche et al., 2016). Flavone C-glycosides are commonly detected as 6-Cand 8 -C-glycosides. The flavone $O$-glycosides can be easily hydrolyzed with enzyme or acid; however, flavone $\mathrm{C}$-glycosides are resistant to both process and must be analyzed in their native forms 
Table 2. Different types of flavonoids based on the position of their substituents

\begin{tabular}{|c|c|c|c|c|c|c|}
\hline Ring position & 3 & 5 & 7 & $3^{\prime}$ & $4^{\prime}$ & $5^{\prime}$ \\
\hline \multicolumn{7}{|l|}{ Flavonols $^{1}$} \\
\hline Kaempferol & $\mathrm{OH}$ & $\mathrm{OH}$ & $\mathrm{OH}$ & $\mathrm{H}$ & $\mathrm{OH}$ & $\mathrm{H}$ \\
\hline Myricetin & $\mathrm{OH}$ & $\mathrm{OH}$ & $\mathrm{OH}$ & $\mathrm{OH}$ & $\mathrm{OH}$ & $\mathrm{OH}$ \\
\hline Quercetin & $\mathrm{OH}$ & $\mathrm{OH}$ & $\mathrm{OH}$ & $\mathrm{OH}$ & $\mathrm{OH}$ & $\mathrm{H}$ \\
\hline \multicolumn{7}{|l|}{ Flavonones $^{1}$} \\
\hline Hesperetin & $\mathrm{H}$ & $\mathrm{OH}$ & $\mathrm{OH}$ & $\mathrm{OH}$ & O-Me & $\mathrm{H}$ \\
\hline Naringin & $\mathrm{H}$ & $\mathrm{OH}$ & O-R & $\mathrm{H}$ & $\mathrm{OH}$ & $\mathrm{H}$ \\
\hline \multicolumn{7}{|l|}{ Flavones $^{1}$} \\
\hline Apigenin & $\mathrm{H}$ & $\mathrm{OH}$ & $\mathrm{OH}$ & $\mathrm{H}$ & $\mathrm{OH}$ & $\mathrm{H}$ \\
\hline Luteolin & $\mathrm{H}$ & $\mathrm{OH}$ & $\mathrm{OH}$ & $\mathrm{OH}$ & $\mathrm{OH}$ & $\mathrm{H}$ \\
\hline \multicolumn{7}{|l|}{ Anthocyanidins $^{2}$} \\
\hline Cyanidin & $\mathrm{OH}$ & $\mathrm{OH}$ & $\mathrm{OH}$ & $\mathrm{OH}$ & $\mathrm{OH}$ & $\mathrm{H}$ \\
\hline Malvidin & $\mathrm{OH}$ & $\mathrm{OH}$ & $\mathrm{OH}$ & $\mathrm{OCH}_{3}$ & $\mathrm{OH}$ & $\mathrm{OCH}_{3}$ \\
\hline Petunidin & $\mathrm{OH}$ & $\mathrm{OH}$ & $\mathrm{OH}$ & $\mathrm{OCH}_{3}$ & $\mathrm{OH}$ & $\mathrm{H}$ \\
\hline \multicolumn{7}{|l|}{ Flavanols $^{3}$} \\
\hline Catechin/Epicatechin & $\mathrm{OH}$ & $\mathrm{OH}$ & $\mathrm{OH}$ & $\mathrm{OH}$ & $\mathrm{OH}$ & $\mathrm{H}$ \\
\hline Gallocatechin & $\mathrm{OH}$ & $\mathrm{OH}$ & $\mathrm{OH}$ & $\mathrm{OH}$ & $\mathrm{OH}$ & $\mathrm{OH}$ \\
\hline Epigallocatechin & $\mathrm{OH}$ & $\mathrm{OH}$ & $\mathrm{OH}$ & $\mathrm{OH}$ & $\mathrm{OH}$ & $\mathrm{OH}$ \\
\hline Epigallocatechin gallate & G & $\mathrm{OH}$ & $\mathrm{OH}$ & $\mathrm{OH}$ & $\mathrm{OH}$ & $\mathrm{OH}$ \\
\hline \multicolumn{7}{|l|}{ Isoflavones ${ }^{3}$} \\
\hline Genistein & - & $\mathrm{OH}$ & $\mathrm{OH}$ & $\mathrm{H}$ & $\mathrm{OH}$ & $\mathrm{H}$ \\
\hline Daidzin & - & $\mathrm{H}$ & O-Glu & $\mathrm{H}$ & $\mathrm{OH}$ & $\mathrm{H}$ \\
\hline Daidzein & $\mathrm{H}$ & $\mathrm{H}$ & $\mathrm{OH}$ & $\mathrm{H}$ & $\mathrm{OH}$ & $\mathrm{H}$ \\
\hline
\end{tabular}

${ }^{1}$ Narayana et al., 2001; ${ }^{2}$ Graf et al., 2005; ${ }^{3}$ Shahidi and Naczk, 2003. -O-Me, Methoxy; -O-Glu, Glucosyl; -O-R, Alkoxy; and G, Gallate.

(Hostetler et al., 2017). Changing the functional R1 and R2 bonds in the flavone core will yield different types of flavones including apigenin, acacetin, luteolin, diosmetin, and chrysoeriol.

Apigenin, also known as 4',5,7-trihydroxyflavone, is present in grapefruit, plant-derived beverages, parsley, celery, onions, oranges, tea, chamomile, wheat sprouts and in some seasonings. The most abundant single source of apigenin is chamomile prepared from dried flowers of Matricaria chamomilla (El Gharras, 2009; Manach et al., 2004; Shukla and Gupta, 2010). Acacetin is an $O$ methylated flavone in its natural form is found in various plants including Agastache rugosa, Scoparia dulcis, Cirsium rhinoceros, Robinia pseudoacacia and Tamarindus indica (Bhat et al., 2013; Daniel, 2006; Liu et al., 2011; Yim et al., 2003; Zhao et al., 2008). Luteolin is found in oregano, celery, orange, broccoli, rosemary, green pepper, peppermint, parsley, olive oil, thyme, carrot, dandelion, chamomile tea, and perilla (Akhtar and Swamy, 2018). Diosmin (3',5,7-trihydroxy-4'-methoxyflavone-7-rutinoside) is commonly found in most plants and fruits especially citrus fruits (Russo et al., 2018). Some of the plants from which diosmetin has been isolated include Acacia farnesiana, Artemisia vulgaris, Chrysanthemum morifolium, Citrus medica, Cyprideis torosa, Daphne pseudomezereum, Ditylenchus acris, Eclipta prostrata, Galium verum, Genus Vicia, Inula Britannica, Lespedeza davurica, Lonicera syringantha, Luffa cylindrical, Luffa cylindrical, Mallotus stenanthus, and Marrubium alysson (Patel et al., 2013). Chrysoeriol is a flavonoid which is found in various plants including peanut hulls, Cynanchum formosanum, Stachys chrysantha, Artemisia vulgaris and Stachys candida, herbs such as Ludwigia prostrata and flowers of Lonicera japonica as well as leaves of Epimedium sagittatum (Mishra et al., 2003). In wheat, tricin (5,7,4-trihydroxy 3,5-dimethoxyflavone) is a main flavone (Shahidi and Ambigaipalan, 2015).

\subsubsection{Flavonols}

Flavonols (Figure 2) are a subclass of flavonoids with a keto group. The richest sources of flavonols include onions, apples, cider, grapes, wine and tea (Shahidi and Ambigaipalan, 2015). They are derived from the simplest flavonol called 3-hydroxyflavone. The most common flavonols include quercetin, kaempferol, myricetin, isorhamnetin, tamarixetin, morin, and fisetin. The most studied and most bioavailable flavanol is quercetin. Kaempferol and myricetin are available in many foods; however, isorhamnetin and tamarixetin are methylated metabolites of quercetin that also occur naturally (Perez-Vizcaino and Duarte, 2010). Quercetin is available in most vegetables in the $O$-glycosidic form at concentrations below $10 \mathrm{mg} / \mathrm{kg}$. In addition, apigenin, quercetin, kaempferol, and 
myricetin are known to have interactions with soyprotein (Rawel et al., 2002; Yeo and Shahidi, 2019).

Quercetin in onions is an exception, containing 185-634 mg/ $\mathrm{kg}$ of fresh weight (Dávalos et al., 2006). Flavonol content of 25 edible berries was studied, and it was reported that quercetin was present in all berries and the highest amount was found in bog whortleberry $(158 \mathrm{mg} / \mathrm{kg}$, fresh weight), followed by lingonberry (74-146 mg/kg fresh weight), chokeberry $(89 \mathrm{mg} / \mathrm{kg}$ fresh weight), sweet rowan (85 mg/kg fresh weight), cranberry (83-121 $\mathrm{mg} / \mathrm{kg}$ of fresh weight), rowanberry $(63 \mathrm{mg} / \mathrm{kg})$, sea buckthorn berry $(62 \mathrm{mg} / \mathrm{kg}$ ), and crowberry (53 and $56 \mathrm{mg} / \mathrm{kg}$ ). The study also indicated that only 8 out of 25 berries had myricetin and 4 out of 25 berries had kaempferol present in them. The highest amount of myricetin was found in cranberry (74-142 $\mathrm{mg} / \mathrm{kg}$ fresh weight) followed by crowberry (44-49 $\mathrm{mg} / \mathrm{kg}$ fresh weight). The highest amount of kaempferol was found in gooseberry $(16-19 \mathrm{mg} / \mathrm{kg}$ fresh weight) (Häkkinen et al., 1999).

\subsubsection{Flavanones}

Flavanones (Figure 2) occur as two types of glycosides including rutinosides (6-O- $\alpha$-L-rhamnosyl-D-glucosides) and neohesperidosides (2-O- $\alpha$-L-rhamnosyl-D-glucosides) and occur as aglycones. The aglycone forms are not attached to sugar moieties such as naringenin, hesperetin, isosakuranetin and eriodictyol. The glycoside forms of flavanones include hesperidin, narirutin, neohesperidin, neoeriocitrin and naringin. Orange, lemon, grapefruit, lime and mandarin are rich sources of flavanones (Di Majo et al., 2005; Tomás-Barberán and Clifford, 2000b).

\subsubsection{Isoflavones}

Isoflavones (Figure 2) are naturally present as glycoside in plants and are polar molecules. They are widely present in various legumes, soy products such as soy oils, soy lecithin, tofu, black beans, green split peas and clover. The concentration of isoflavones in soy foods range between 0.1 and $3.0 \mathrm{mg} / \mathrm{g}$ (Setchell and Cassidy, 2018). The aglycone forms of isoflavones are daidzein and genistein. The glycosidic form of isoflavones include acetyl and malonyl glucosides and $\beta$-glucosides of daidzein and genistein (Pietta et al., 2003). The germination of soybean was reported to change its isoflavone profile and resulting in a better antioxidant activity in the products than in soybean itself (Yoshiara et al., 2018).

\subsubsection{Anthocyanidins and anthocyanins}

Anthocyanidins (Figure 2) are aglycone derivatives of 3,5,7-trihydroxyflavylium chloride and the most common types of anthocyanidins are cyanidin, delphinidin, pelargonidin, peonidin, petunidin, and malvidin. Anthocyanidins are found very rarely in fresh plants due to their instability. Anthocyanins also called glycosylated anthocyanidins are water-soluble pigments present in flowers and fruits of many plants. Most of the red, purple and blue-colored flowers contain anthocyanins. Red flowers such as red hibiscus, red rose, red pineapple sage, red clover and pink blossom contain anthocyanins and are edible. Blue flowers such as cornflower, blue chicory and blue rosemary and purple flowers such as purple mint, purple passionflower, purple sage, common violet and lavender are the common edible flowers. Some of the fruits containing anthocyanins include cherries, plums, strawberries, raspberries, blackberries, grapes, red currants and black currants (Shahidi and<smiles>Oc1cc(O)c2c(c1)O[C@H](c1ccc(O)c(O)c1)[C@H](O)[C@H]2c1c(O)cc(O)c2c1O[C@H](c1ccc(O)c(O)c1)[C@H](O)[C@H]2c1c(O)cc(O)c2c1O[C@H](c1ccc(O)c(O)c1)[C@H](O)C2</smiles>

Procyanidins: $\mathbf{n}>0$; Oligomeric procyanidins: $\mathbf{n}=0-7$

Figure 3. Structures of proanthocyanidins.

Ambigaipalan, 2015; Valdez and Bolling, 2019). Stability of anthocyanins is dependent on the type of anthocyanin pigment, copigments, metal ions, light, $\mathrm{pH}$, temperature, oxygen, enzymes and antioxidants (Castañeda-Ovando et al., 2009; Khoo et al., 2017; Pietta et al., 2003).

\subsubsection{Tannins}

\subsubsection{Condensed tannins: flavan-3-ols (or) flavanols or proan- thocyanidins}

Flavanols (Figure 2) are like 4-oxo flavonoids but are characterized by the lack of double bond between $\mathrm{C} 2$ and $\mathrm{C} 3$, and no $\mathrm{C} 4$ carbonyl in ring $\mathrm{C}$ of flavanols. In addition, hydroxylation at $\mathrm{C} 3$ allows flavanols to have two chiral centres on $\mathrm{C} 2$ and $\mathrm{C} 3$, thus having four possible diastereoisomers. Naturally, flavanols are found as catechin and epicatechin isomers. Catechin is the isomer with trans configuration and epicatechin is the one with cis configuration. Flavanols are present in apples, apricots, pears, cherries, peaches and plums (Pietta et al., 2003; Tsao, 2010). Proanthocyanidins (Figure 3) include oligomeric and polymeric forms of monomeric flavanols (Shahidi et al., 2019). Proanthocyanidins are also called condensed tannins, and are distinguished according to the substitution pattern of their linking. The A-type proanthocyanidins are linked through $\mathrm{C} 2-\mathrm{O}-\mathrm{C} 7$ or $\mathrm{C} 2-\mathrm{O}-\mathrm{C} 5$ bonding, B-type proanthocyanidins are linked through $\mathrm{C} 4-\mathrm{C} 6$ or $\mathrm{C} 4-\mathrm{C} 8$ bonding as dimers. There are two types of proanthocyanidins, namely procyanidins and prodelphidins (Tsao, 2010).

\subsubsection{Hydrolyzable tannins}

Hydrolyzable tannins (Figure 4) are compounds that are intermediate- to high-molecular- weight phenolics which can weigh up to $30,000 \mathrm{Da}$. They are composed of esters of gallic acid (gallotannins) or ellagic acid (ellagitannins) with a glucose core and are readily hydrolyzed by acids or enzymes into monomeric products 
<smiles>O=C(O)c1cc(O)c(O)c(O)c1</smiles>

Gallic acid<smiles>COC1=C(O)C=C2C=C(O)C(=C1)C(OC(=O)c1cc(O)c(O)c(O)c1)C(OC(=O)c1cc(O)c(O)c(O)c1)C(OC(=O)c1cc(O)c(O)c(O)c1)C(OC(=O)c1cc(O)c(O)c(O)c1)COC2=O</smiles>

Gallotannin

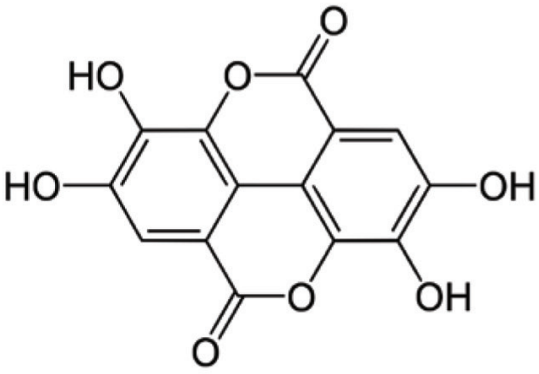

Ellagic acid<smiles>O=C(O)c1cc(O)c(O)c(O)c1-c1c(C(=O)O)cc(O)c(O)c1O</smiles>

Hexahydroxydiphenic acid

Figure 4. Structure of hydrolyzable tannins and their monomeric compounds.

(Grasel et al., 2016; Pietta et al., 2003; Shahidi et al., 2019).

\subsubsection{Miscellaneous phenolic compounds}

The minor dietary components are compounds in which some are restricted to botanical occurrence whereas others are widespread among nuts and fruits. The biological significance and metabolism of some these compounds are relatively unknown. Simple phenols like arbutin (hydroquinone or quinol $\beta$-D-glucoside) are found in pears, strawberry tree, and breaberry. Meanwhile, sesamolin (1,2-methylenedioxy-4-hydroxybenzene) is present in sesame seed and oil. Derivatives of 4-ethylresorcinol such as 5-alkyl resorcinols, isomeric 5-alkenyl resorcinols and 5-alkadienyl resorcinols found in whole wheat grains, rice and rye are also a part of minor food metabolites. Urushiol member of Anacardiaceae family is found in mango skins and Australian cashew. Anacardic acid, also called as 6-pentadec(en)ylsalicylic acids, is found in cashew nut and apple. Smoke phenols are compounds that are released during smoking of wood and the precise composition of wood smoke depends on the type of wood and wood combustion temperature. The content of simple phenols in smoked foods include phenols, cresols, xylenols, guaiacol (2-methoxyphenol), eugenol, 4-ethylguaiacol, carvacrol, and syringol (2,6-dimethoxyphenol) (Budowski, 1964; Chakraborty et al., 1998; Funayama et al., 1995; Issenberg et al., 1971; Jeng and Hou, 2005; Kang et al., 1998; Kubo et al., 2006; Maeda and Fukuda, 1996; Mullin and Emery, 1992; Wang et al., 2018).

Lignans are plant-derived compounds and are found in whole grain products, vegetables, fruits, nuts, seeds and beverages such as tea, coffee and wine. Lignans are dimers of phenylpropane units (Shahidi et al., 2019). There are two classes of phenylpropanoids including allyl (methyl-vinyl, $\mathrm{R}-\mathrm{CH}_{2}-\mathrm{CH}=\mathrm{CH}_{2}$ ) and propenyl (vinyl-methyl, $\mathrm{R}-\mathrm{CH}=\mathrm{CH}-\mathrm{CH}_{3}$ ). Some of the known phenylpropanoids are safrole (1-allyl-3,4-methylenedioxybenzene), estragole (1-allyl-4-methoxybenzene), myristicin (1-allyl-3,4-methylenedioxy-5-methoxybenzene), eugenol (1-allyl-3-methoxy-4-hydroxybenzene) and trans-anaethole (Clifford, 2000; Kumano et al., 2016).

Coumarins are a class of lactones which are fused by a benzene ring to $\alpha$-pyrone ring. They are also called benzopyrones $(1,2$-benzopyrones or 2H-1-benzopyran-2-ones) and widely distributed in the nature. Coumarins were first isolated from Tonka beans, found in more than 1,300 plant species and distributed in more than 40 different families. Coumarins are well distributed in Angiospermae, Monocotyledoneae, Dicotyledoneae, Apiceae, Rutaceae, Asteraceae, Fabaceae, Oleaceae, Moraceae and Thymelaeaceae families. Coumarins are subdivided into simple coumarins, furanocoumarins, dihydrofuranocoumarins, pyranocoumarins, phenylcoumarins and bicoumarins. Pyranocoumarins are divided into two types present in the angular and linear forms (Clifford, 2000; Matos et al., 2015). 
Quinones are rare components in the diet and are found in plants, fungi and bacteria. They play a significant role as electron transport chains involved in cellular respiration and photosynthesis. Quinones in nature are biosynthesized via shikimate or polyketide pathways, mammals can synthesize quinones primarily through oxidative metabolism of aromatic precursors such as catecholamines, estrogens and xenobiotics. Quinones can occur in a variety of forms including monocyclic (ex: benzoquinone), extended (ex: ethylstilbestrone) or condensed (ex: benzo $[\alpha]$ pyrene-1,6-dione) (Monks et al., 1992). Complex ranges of monomeric and dimeric anthraquinones are reported to be produced from rhubarb. Some of these compounds include chrysophanol, emodin, aloe-emodin and rhein. Walnut fruit contains 1,4,5-trihydroxynaphthalene-4$\beta$-D-glucoside which acts as a raw material to produce juglone (5-hydroxy-1,4-naphthoqinone) during ripening by hydrolysis and oxidation. This quinone is responsible for the yellow-brown staining and irritation of the hands that can occur after handling these nuts (Clifford, 2000).

Some of the other minor phenolic compounds present in the food include curcuminoids (present in ginger and turmeric), capsaicins (chillies and gingers) and terpenoid phenols such as thymol (4-isopropylphenol), carnosic acid, carnosol, rosmanol, rosmariquinone and rosmaridiphenol (present in oregano, rosemary, sage, thyme). Several marine algae contain various phenols, halogenated phenols (red algae) and pholortannins (brown algae) (Clifford, 2000).

\subsection{Bioavailability of phenolic compounds}

Flavonoids under natural conditions exist in the form of glycosides, linked to various sugar moieties, and are poorly absorbed in the gut. The sugar linked to them is usually glucose or rhamnose but can also be galactose, arabinose, xylose, glucuronic acid and other sugars. For the flavonoids to diffuse through the small intestinal brush border, removal of the hydrophilic sugar moiety is essential. In order to be more bioavailable, these compounds need to be hydrolyzed to their aglycone forms by enzymes like $\alpha$-glucosidase found in the intestinal flora. Therefore, the enzyme specificity and distribution play a vital role in flavonoid absorption. In most cases, it is reported that in brush borders of intestine and absorption in the liver, flavonoids undergo phase II metabolism to form various glucuronides, sulfates or methylated derivatives (Koutsos et al., 2015; Russo et al., 2018; Scalbert and Williamson, 2000). The small intestine directly absorbs low-molecular-weight flavonoids; however, high-molecular-weight flavonoids reach the colon almost unchanged, and they are hydrolyzed by intense metabolic activity before absorption. The microorganisms present in the colon catalyze the breakdown of flavonoids into simpler compounds such as phenolic acids. The absorption of flavonoids is affected by various factors including molecular size, the degree of polymerization, solubility, food matrix, interaction with other compounds, gut microbiota composition and passage through the gastrointestinal tract (Koutsos et al., 2015; Scalbert and Williamson, 2000; Shahidi and Peng, 2018). The mechanism behind flavonoid metabolism and absorption is still not fully elucidated due to the complexity of numerous polyphenol compounds and a plethora of gut microorganisms present in the human gut. The knowledge present in the literature is based on polyphenols and fewer gut microbiota. To better understand, the polyphenol-microbial interaction and metabolism, new technologies such as metagenomics and metatranscriptomics can be used extensively in the future (Kemperman et al., 2010). The process of polyphenol bioavailability, metabolism and absorption is shown in Figure 5.

Phenolic compounds when ingested as a part of food matrix undergo various stages of absorption and metabolism processes before exhibiting bioactivities associated with them. In the first step, the phenolic compounds are released from the food matrix by mechanical actions such chewing and grinding in oral activity along with enzymatic action of various digestive enzymes present in saliva. The phenolic compounds must endure various $\mathrm{pH}$ changes and they are transported to stomach, where they are subjected to various digestive enzymes such as pepsin, trypsin, esterases, lipases and amylases and gastric juice. The phenolic compounds are then subjected to various metabolic activities such as Phase I, Phase II and Phase III metabolism as part of the default detoxification system. During Phase I metabolism, cytochrome p450 (CYP) superfamily of enzymes hydroxylates incoming phenolic compounds (De Montellano, 2005; Guengerich, 2007; Shahidi and Peng, 2018). This step is a functionalization step which makes phenolic compounds more reactive for subsequent metabolism. During phase II metabolism, the phenolic compounds undergo conjugation process in which hydroxyl group is modified by the addition of sulfate, glucuronic acid or a methyl group (Shahidi and Peng, 2018). Glucuronidation process is carried out by uridine diphosphate glucuronyl-transferase (UDPGT), methylation of phenolic compounds is performed by catechol $O$-methyl transferase (COMT), and sulphation is carried out by sulfotransferase (SULT) or by phenol sulfotransferase (PST) (Heleno et al., 2015). The conjugated phenolic compounds are transported into interstitial space and bloodstream by basolateral transporter such as multidrug resistant protein (MRP) 1 and circulated into the liver via the portal vein. The phenolic compounds also undergo extensive phase II metabolism mainly with the help of COMT. The metabolism happening inside the liver is also called first pass metabolism because this is where the absorbed phenolic compounds compounds are first exposed to metabolism before entering blood circulation (Rio et al., 2010). The metabolism compounds enter circulation and are absorbed by the tissues and transported into kidneys and excreted via urine. The number of phenolic compounds excreted via urine is relatively low, and it indicates that most of the phenolic compounds are transformed in the gut and excreted via feces (Tulipani et al., 2012). Some unabsorbed phenolic compounds in the liver are effluxed into the intestinal lumen via luminal (apical) transporters such as P-glycoprotein (Pgp) and multidrug resistant protein (MRP) 2 thereby reducing the net absorption in the liver. This active efflux of polyphenols into the intestinal lumen is called Phase III metabolism (Xu et al., 2005). In addition, some metabolites from liver are effluxed from liver into bile and transported into intestinal lumen for subsequent metabolism or excretion via feces (Xu et al., 2009; Shahidi and Peng, 2018).

In the first step of colonic metabolism, the microbial deconjugation of glucuronides and sulfates into aglycones are carried out by plethora of microorganisms. Aglycones, monomers to trimers of phenolic compounds and some intact glycosides such as anthocyanins can be rapidly absorbed by both enterocytes and colonocytes. The aglycones are then converted into various metabolites and are absorbed in the colonocytes and the rest re-excreted via feces (Kemperman et al., 2010; Neilson et al., 2017; Viskupičová et al., 2008).

\subsection{Metabolism of phenolic acids}

Phenolic acids are considered as secondary metabolites from plants and fungi. These compounds are produced for protection against UV light, insects, viruses and bacteria (Naczk and Shahidi, 2006). Phenolic acids are divided into two major groups: hydroxybenzoic acid and hydroxycinnamic acids. The two types of phenolic ac- 


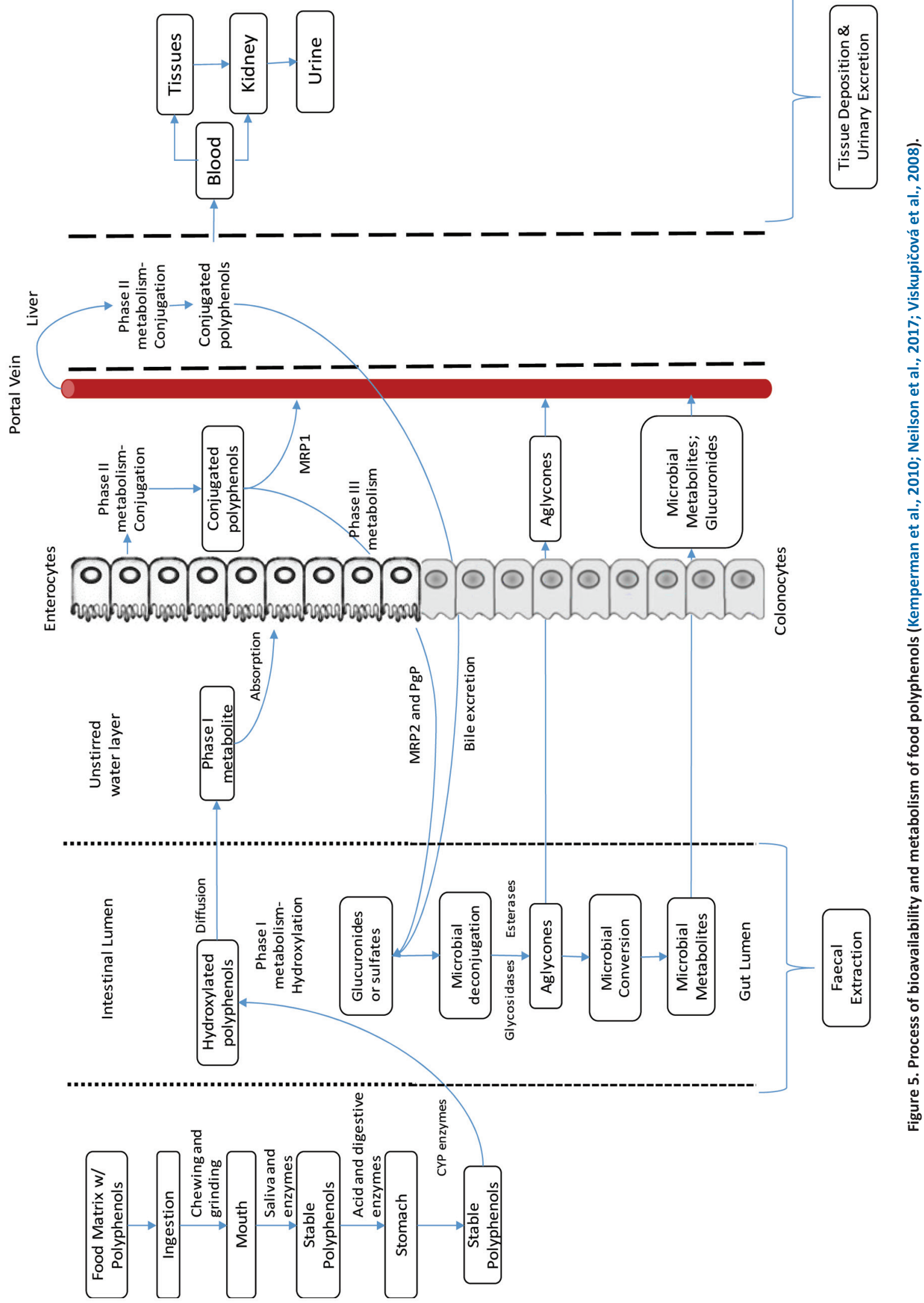


ids are differentiated by the substitution in the functional groups such as hydrogen, hydroxyl and methoxy groups determining their functional property and bioactivity. It is also reported that phenolic acids are the main bound components in various food sources such as cereal grains, legumes and other seeds, acting as building material for the cell wall matrix (Heleno et al., 2015; Benevides et al., 2018; Shahidi and Yeo, 2016). Phenolic acids are synthesized via shikimate pathway essentially from phenylalanine or, to a lesser extent, tyrosine. The phenolic acids undergo various metabolic processes such as deamination, hydroxylation, oxidation and methylation corresponding derivatives (Shahidi and Yeo, 2018). Degradation of benzoic acid produces various metabolites such as $p$-hydroxybenzoic acid, protocatechuic acid, vanillic acid, veratric acid, gallic acid, genistic acid and syringic acid. Degradation of cinnamic acid produces various metabolites such as $p$-coumaric acid, caffeic acid, ferulic acid, sinapic acid and 3,4- or 5-O-caffeoylquinic acid. Lafay and Gil-Izquierdo (2008) reported when phenolic acids are in the aglycone form, they are absorbed in the upper part of the gastro-intestinal tract and stomach is also found to be an active absorption site. Phenolic acids such as gallic, caffeic, ferulic, coumaric and chlorogenic acids are absorbed in the stomach. Generally, the absorption of phenolic acids is very rapid between $1-2 \mathrm{~h}$ after food intake. However, esterified forms of phenolic acids are less bioavailable and only $0.3-0.4 \%$ are absorbed from the original food intake. The lower bioavailability of the phenolic acids is because esterified phenolic acids must be hydrolyzed in the enterocytes before reaching the blood circulation and the enzymatic capacity of the intestinal cells is not so efficient to hydrolyze the ester bonds. These unabsorbed esterified phenolic acids are metabolized by microflora present in the colon.

\subsubsection{Ferulic acid and caffeic acid}

Ferulic acid and caffeic acid are the main hydroxycinnamic acid derivatives. Ferulic acid can be found in grains and vegetables, whereas caffeic acid is a major phenolic acid in sunflower seeds (Chen and Ho, 1997). Pereira-Caro et al. (2016) studied the presence of phenolic compounds in the plasma and urine after the consumption of orange juice. During this study, the HPLC-HRMS results indicated presence of benzoic acid derivatives such as 3,4-dihydroxybenzoic acid, hydroxybenzoic acid- $O$-glucuronide, 3-hydroxybenzoic acid-4-sulfate, 4-hydroxybenzoic acid-3 sulfate, 3-methoxy-4-hydroxybenzoic acid, 3-hydroxy-4-methoxybenzoic acid, 3-hydroxybenzoic acid, 4-hydroxybenzoic acid, 4'-hydroxycinnamic acid and dihydroferulic acid which could be derived from ferulic acid.

Rechner et al. (2001) studied the novel biomarkers of the bioavailability and metabolism of hydroxycinnamate derivatives through the determination of the pharmacokinetics of their urinary elimination. Coffee was used as the main dietary source of caffeic acid in this testing. The in vivo experiments were carried out on five healthy male volunteers with pre-study washout procedure for $24 \mathrm{~h}$ and on the study day, 2 cups of coffee were provided with polyphenol free diet for $24 \mathrm{~h}$. The urine samples collected every $4 \mathrm{~h}$ were tested for the presence of metabolites after coffee consumption. The results indicated presence of various metabolites including ferulic, isoferulic, dihydroferulic acid, (3-(4-hydroxy3-methoxyphenyl)-propionic acid), 3-methoxy-4-hydroxybenzoic acid, hippuric acid, 3-hydroxyhippuric acid and vanillic acid in urine in significantly higher levels compared to the pre-supplementation levels.

Crozier et al. (2010) reported that caffeic acid, ferulic acid, and $p$-coumaric acid are hydroxycinnamates linked to quinic acid to form a range of conjugated structures known as caffeoylquinic acids, feruloylquinic acids and $p$-coumaroylquinic acids in isomeric forms. In addition, it is reported that small intestine is most probably the site for the breakdown of caffeoylquinic acids and feruloylquinic acids to release quinic acid, caffeic acid and ferulic acid as the products. Furthermore, sulfation and glucuronidation of caffeic acid and ferulic acid, methylation of caffeic acid to isoferulic acid are metabolized in the small intestine. The colonic metabolism is evident in the conversion of ferulic acid to feruloylglycine and dihydroferulic acid and metabolism of caffeic acid to dihydrocaffeic acid which is further metabolized to dihydro-isoferulic acid.

\subsubsection{Gallic acid and protocatechuic acid}

Gallic acid, a hydroxybenzoic acid is available in plants in the free or esterified form and is distributed in large amount in tea leaves (Shahrzad et al., 2001). Zong et al. (1999) studied the metabolism of gallic acid orally administered in rats and found that gallic acid metabolized to produce 4-O-methyl gallic acid and the maximum concentration was found in portal vein and inferior vena cava at 15 and 30 min after administration. Also, they observed that the only 4-O-methylgallic acid was seen in the liver but not gallic acid. Analysis of blood samples in this study indicated that gallic acid and 4-O-methylgallic acid were 100 times lower than that found in urine samples. This indicated that most of the gallic acid was excreted out in urine without being metabolized. The study also indicated that gallic acid administered orally existed in the blood for $6 \mathrm{~h}$ and half of it was metabolized as 4-O-methylgallic acid. The study concluded that since relatively high concentrations of gallic acid was present in the portal vein and remained longer when administered orally compared to intravenous or intraperitoneally it should have significant consideration of being used as an anticancer drug treating liver cancer.

Konishi et al. (2004) studied the intestinal absorption of $p$-coumaric acid and gallic acids in rats after oral administration. Generally, ferulic acid and $p$-coumaric acid are absorbed by the monocarboxylic acid transporter, however gallic acid is not absorbed via that manner. Therefore, the pharmacokinetic profiles and the absorption of and $p$-coumaric acid and gallic acid after oral administration was carried out. In this study, the rats were administered with $100 \mu \mathrm{mol} / \mathrm{kg}$ body weight of $p$-coumaric acid and gallic acid then the blood was collected from the portal vein as well as abdominal artery. The results indicated that $p$-coumaric acid rapidly absorbed in the gastrointestinal tract in an intact form with the concentrated peak in portal vein at $10 \mathrm{~min}$ after dosing. In contrast, the study revealed that gallic acid was slowly absorbed and intact form with concentrated peak at portal vein at $60 \mathrm{~min}$. The serum concentration profile was $p$-coumaric acid and gallic acid in the portal vein was reported to $2,991.3$ and $42.6 \mu \mathrm{mol} \mathrm{min} / \mathrm{L}$, respectively. The relative bioavailability of $p$-coumaric acid against gallic acid was about 70. Konishi et al. (2006) studied the absorption rates of various phenolic acid in the rat stomach and the results showed that five min after administration of $2.25 \mu \mathrm{mol}$ of various phenolic acids in the rat stomach, the plasma concentration of each phenolic acid increased in the order of: gallic acid $=$ chlorogenic acid $<$ caffeic acid $<p$-coumaric acid $=$ ferulic acid.

Konishi et al. (2003) studied the transepithelial transport of $p$ coumaric acid and gallic acid in Caco-2 cell monolayers. The results exhibited that the transport of coumaric acid was dependent on $\mathrm{pH}$ and in a vectorial manner in the apical-basolateral direction. However, the results indicated that unlike coumaric acid, the permeation of gallic acids was not polarized and was independent of 
$\mathrm{pH}$ and linearity increased with increasing concentration of gallic acid. Furthermore, the transport rate of gallic acid was about 100 times lower than that of coumaric acid suggesting paracellular pathway.

Serra et al. (2012) studied the colonic metabolism of gallic and protocatechuic acid using rat microflora. Both phenolic acids were metabolized by colonic microflora. There was an increase in all the metabolites throughout the fermentation process from $0-48 \mathrm{~h}$ period. The main metabolites of gallic acid were phenylacetic acid and $p$-hydroxyphenylacetic acid at 15 and $16 \mathrm{pM}$ concentrations, respectively. The results also indicated that gallic acid was also present throughout the fermentation process from $43 \mathrm{pM}$ at $0 \mathrm{~h}$ to $28 \mathrm{pM}$ at $48 \mathrm{~h}$. The main metabolites of protocatechuic acid fermentation were phenylacetic acid and $p$-hydroxybenzoic acid at 39 and $44 \mathrm{pM}$ concentrations, respectively. The results also showed that protocatechuic acid was also present throughout the whole fermentation process from $296 \mathrm{pM}$ at $0 \mathrm{~h}$ to $254 \mathrm{pM}$ at $48 \mathrm{~h}$. The poor metabolism was due to the presence of monophenol structures as metabolic products of these polyhydroxy polyphenol compounds like flavonoids.

\subsection{Metabolites of polyphenols}

There are many studies reporting that much of the polyphenol metabolites are poorly absorbed in the small intestine and liver (Shahidi and Peng, 2018). However, the metabolic activity of the gut microflora on unabsorbed polyphenols plays an important role in the modulation of bioactivities of these compounds and their health effects. Furthermore, various studies have demonstrated that these polyphenols are transformed into various phenolic acids such as phenylvaleric, phenylpropionic, phenylacetic, benzoic and hippuric acids. However, the type of metabolic products depends upon the type of compound metabolized (Gonthier et al., 2018). Therefore, it is essential that we consider all changes that occur upon digestion and all these must be carefully considered when we speak about absorption and bioavailability.

Dall'Asta et al. (2012) studied the identification of microbial metabolites derived from in vitro fecal fermentation of 16 different food sources: fresh strawberries, blueberries, raspberries, blackberries, Tarocco blood orange, onion, clarified apple juice, pomegranate juice, dark chocolate $(90 \%$ cocoa), red wine (Merlot), oat bran, wheat bran, flaxseeds, black and green tea, and ground coffee. The polyphenols present in the foods were extracted using various methods and was stored at $-80{ }^{\circ} \mathrm{C}$ until in vitro fermentation. The fermentation was carried out in a total volume of $22 \mathrm{~mL}$ containing $45.5 \%$ growth medium, $45.5 \%$ fecal slurry and $9 \%$ food sample matrix. The fecal slurry was obtained from three healthy volunteers who followed polyphenol-free diet for 2 days before fecal collection. The fermentation was carried out at $37^{\circ} \mathrm{C}$ for 24 $\mathrm{h}$. The samples were collected at $5 \mathrm{~h}$ and $24 \mathrm{~h}$ time intervals. The phenolic compounds originally present in food samples and the fermentation derived phenolic metabolites were analyzed using LC/MS. The study demonstrated various metabolites from the in vitro fermentation of all 16 foods in detailed manner are shown in Table 3.

\subsubsection{Metabolism of flavonols}

\subsubsection{Metabolism of quercetin}

Quercetin exists in wide variety of vegetables and fruites such as onions, kale and apples (Formica and Regelson, 1995; Shahidi and Ambigaipalan, 2015). Serra et al. (2012) studied the metabolic fermentation of quercetin, quercetin-rhamnoside and quercetin-rutinoside using rat colonic fermentation for $48 \mathrm{~h}$. The fermentation process was started at a very low starting concentration of $1 \mathrm{pM}$ for quercetin compared to 7.3 and $23 \mathrm{pM}$ of quercetin-rhamnoside and quercetin-rutinoside, respectively. The results from this study indicate that there was immediate degradation of quercetin with the formation of various metabolites such as phenylacetic acid, three different mono hydroxylated forms ( $p-, m$ , and $o$-hydroxyphenylacetic acid) and 3,4-dihydroxyphenylacetic acid, protocatechuic acid, 3-(3,4-dihydroxyphenyl)propionic acid, homovanillic acid (2-(4-hydroxy-3-methoxyphenyl)acetic acid), and $p$-hydroxybenzoic acid. The highest concentration of quercetin metabolite was protocatechuic acid reaching a maximum of $63 \mathrm{pM}$ after $24 \mathrm{~h}$ incubation, followed by phenylacetic acid, which reached a concentration of $42 \mathrm{pM}$ after incubation for $48 \mathrm{~h}$. In the same period, the concentration of quercetin fluctuated and reached $0.33 \mathrm{pM}$ after $24 \mathrm{~h}$ but was not detectable after $48 \mathrm{~h}$ incubation. When quercetin-rhamnoside was fermented with rat colonic microflora, the main metabolic product was phenylacetic and $p$-hydroxyphenylacetic acid reaching a concentration of 41 and $34 \mathrm{pM}$, respectively. The results also indicate that there was a much faster degradation of quercetin-rhamnoside reaching a concentration of $2 \mathrm{pM}$ within $4 \mathrm{~h}$ and was almost barely detectable in the system after $24 \mathrm{~h}$ of incubation. Quercetin-rutinoside fermentation using rat microflora resulted in a lower number of metabolites compared to quercetin and quercetin-rhamnoside. The main metabolite of quercetin-rutinoside was 3,4-dihydroxyphenylacetic acid reaching a concentration of $51 \mathrm{pM}$ after $48 \mathrm{~h}$ of incubation, however, it was not seen in the medium for the first $47 \mathrm{~h}$. Similar to quercetin-rhamnoside, quercetin-rutinoside also degraded quickly and was near zero after $24 \mathrm{~h}$ of incubation (Serra et al., 2012).

The in vivo quercetin metabolites identified from body fluids include 2-(3,4-dihydroxyphenyl)acetic acid (human urine), 2-(3-hydroxyphenyl)acetic acid (human serum), 3,4-dihydroxytoluene and 2-(3-methoxy-4-hydroxyphenyl)acetic acid. Generally, flavonoids undergo various transformations caused by microbiota such as ring-fission. During the process of ring fission, the C-rings of the flavonoids is degraded resulting in the formation of hydroxylated aromatic compounds from the A ring and phenolic acid from the $\mathrm{B}$ ring. The primary metabolite of quercetin is 2-(3,4-dihydroxyphenyl)acetic acid, and myricetin metabolism results in 2-(3,5-dihydroxyphenyl) acetic acid. Further, dihydroxylation caused by microbiota results in the formation of 2-(3-hydroxyphenyl)acetic acid from both dehydroxylated derivatives (Aura, 2008).

Mullen et al. (2008) studied the bioavailability of $\left[2-{ }^{14} \mathrm{C}\right]$ quercetin-4'-glucoside in rats over $72 \mathrm{~h}$ period. Radiolabeled products in body tissues, plasma, feces, and urine were monitored by HPLC-PDA-RC-MS/MS. The rats were ingested $\left[2-{ }^{14} \mathrm{C}\right]$ quercetin-4'-glucoside with $18.4 \times 10^{6}$ disintegrations per minute $(\mathrm{dpm})$, weighing $1.0 \mathrm{mg}$. The rats were sacrificed at various time intervals $1,6,12,24,48$, and $72 \mathrm{~h}$ after feeding the radiolabeled $\left[2-{ }^{14} \mathrm{C}\right]$ quercetin-4'-glucoside. The distribution of radioactivity in tissues, plasma, urine, feces, and the cage washings, which consisted of a mixture of urine and feces, was determined. The results indicated that intact form and several metabolites such as quercetin glucuronide, quercetin diglucuronide, methylquercetin glucuronide, methylquercetin diglucuronide, methyl sulfate glucuronide along with 5 phenolic acids were found. The five phenolic acids included 3,4-dihydroxybenzoic acid, 3,4-dihydroxyphenylacetic acid, 3-hydroxyphenylacetic acid, hippuric acid, and benzoic acid. The study revealed that after $1 \mathrm{~h}$ ingestion, almost $41 \%$ of 
Table 3. Metabolites found in in vitro colonic fermented food matrix

\begin{tabular}{|c|c|c|c|}
\hline Food sample & Metabolites & Parent ion $[\mathrm{M}-\mathrm{H}]^{-}(\mathrm{m} / \mathrm{z})$ & Fragment ions $(\mathrm{m} / \mathrm{z})$ \\
\hline \multirow[t]{2}{*}{ Raspberries } & protocatechuic acid & 153 & 109 \\
\hline & benzoic acid & 121 & 77 \\
\hline \multirow[t]{6}{*}{ Blueberries } & gallic acid & 169 & 125 \\
\hline & coumaric acid & 163 & 119 \\
\hline & protocatechuic acid & 153 & 109 \\
\hline & quinic acid & 191 & 85 \\
\hline & dihydrocaffeic acid & 181 & 59,137 \\
\hline & hydroxybenzoic acid & 137 & 93 \\
\hline Blackberries & protocatechuic acid & 153 & 109 \\
\hline \multirow[t]{2}{*}{ Strawberries } & gallic acid & 169 & 125 \\
\hline & quinic acid & 191 & 85 \\
\hline \multirow[t]{2}{*}{ Onion } & (3,4-dihydroxyphenyl)acetic acid & 167 & 123 \\
\hline & phloroglucinol & 125 & 51 \\
\hline \multirow[t]{2}{*}{ Oat bran } & dihydroferulic acid & 195 & 136 \\
\hline & dihydrosinapic acid & 225 & 151 \\
\hline \multirow[t]{2}{*}{ Wheat bran } & dihydroferulic acid & 195 & 136 \\
\hline & dihydrosinapic acid & 225 & 151 \\
\hline \multirow[t]{6}{*}{ Flaxseed } & protocatechuic acid & 153 & 109 \\
\hline & dihydroferulic acid & 195 & 136 \\
\hline & homovanillic acid & 181 & 137 \\
\hline & enterodiol & 301 & 253 \\
\hline & enterolactone & 297 & 253 \\
\hline & dihydrocaffeic acid & 181 & 59,137 \\
\hline \multirow[t]{5}{*}{ Dark chocolate } & 5-(3',4'-dihydroxyphenyl)- $\gamma$-valerolactone & 207 & 163 \\
\hline & (3,4-dihydroxyphenyl)acetic acid & 167 & 123 \\
\hline & protocatechuic acid & 153 & 109 \\
\hline & hydroxybenzoic acid & 137 & 93 \\
\hline & salicylic acid & 137 & 93 \\
\hline \multirow[t]{3}{*}{ Orange juice } & dihydroferulic acid & 195 & 136 \\
\hline & sinapic acid & 223 & 149,208 \\
\hline & protocatechuic acid & 153 & 109 \\
\hline \multirow[t]{4}{*}{ Apple juice } & quinic acid & 191 & 85 \\
\hline & 5-(3',4'-dihydroxyphenyl)- $\gamma$-valerolactone & 207 & 163 \\
\hline & dihydrocaffeic acid & 181 & 59,137 \\
\hline & protocatechuic acid & 153 & 109 \\
\hline \multirow[t]{5}{*}{ Pomegranate juice } & gallic acid & 169 & 125 \\
\hline & pyrogallol & 125 & 51,41 \\
\hline & phlorogucinol & 125 & 51 \\
\hline & syringic acid & 197 & 153,182 \\
\hline & protocatechuic acid & 153 & 109 \\
\hline
\end{tabular}


Table 3. Metabolites found in in vitro colonic fermented food matrix - (continued)

\begin{tabular}{|c|c|c|c|}
\hline Food sample & Metabolites & Parent ion $[\mathrm{M}-\mathrm{H}]^{-}(\mathrm{m} / \mathrm{z})$ & Fragment ions $(\mathrm{m} / \mathrm{z})$ \\
\hline \multirow[t]{11}{*}{ Black tea } & phlorogucinol & 125 & 51 \\
\hline & pyrogallol & 125 & 51,41 \\
\hline & coumaric acid & 163 & 119 \\
\hline & gallic acid & 169 & 125 \\
\hline & 5-(3',4'-dihydroxyphenyl)- $\gamma$-valerolactone & 207 & 163 \\
\hline & $5-\left(3^{\prime}, 4^{\prime}, 5^{\prime}\right.$-trihydroxyphenyl)- $\gamma$-valerolactone & 223 & 179 \\
\hline & 5-(3'-hydroxyphenyl)- $\gamma$-valerolactone & 191 & 147 \\
\hline & protocatechuic acid & 153 & 109 \\
\hline & dihydrocaffeic acid & 181 & 59,137 \\
\hline & homovanillic acid & 181 & 137 \\
\hline & quinic acid & 191 & 85 \\
\hline \multirow[t]{7}{*}{ Coffee } & caffeic acid & 179 & 135 \\
\hline & dihydroferulic acid & 195 & 136 \\
\hline & quinic acid & 191 & 85 \\
\hline & dihydrocaffeic acid & 181 & 59,137 \\
\hline & ferulic acid & 193 & 134 \\
\hline & protocatechuic acid & 153 & 109 \\
\hline & hydroxybenzoic acid & 137 & 93 \\
\hline \multirow[t]{9}{*}{ Green tea } & phloroglucinol & 125 & 51 \\
\hline & pyrogallol & 125 & 51,41 \\
\hline & gallic acid & 169 & 125 \\
\hline & 5-(3',4'-dihydroxyphenyl)- $\gamma$-valerolactone & 207 & 163 \\
\hline & 5-( $\left(3^{\prime}, 4^{\prime}, 5^{\prime}\right.$-trihydroxyphenyl)- $\gamma$-valerolactone & 223 & 179 \\
\hline & protocatechuic acid & 153 & 109 \\
\hline & dihydrocaffeic acid & 181 & 59,137 \\
\hline & 5-(3'-hydroxyphenyl)- $\gamma$-valerolactone & 191 & 147 \\
\hline & quinic acid & 191 & 85 \\
\hline \multirow[t]{8}{*}{ Red wine } & 5-(3',4'-dihydroxyphenyl)- $\gamma$-valerolactone & 207 & 163 \\
\hline & 3-(3-hydroxyphenyl) propionic acid & 165 & 121 \\
\hline & protocatechuic acid & 153 & 109 \\
\hline & gallic acid & 169 & 125 \\
\hline & pyrogallol & 125 & 51,41 \\
\hline & phloroglucinol & 125 & 51 \\
\hline & coumaric acid & 163 & 119 \\
\hline & quinic acid & 191 & 85 \\
\hline
\end{tabular}

Data extracted from Dall'Asta et al. (2012).

the radioactivity remained inside the stomach as un-metabolized substrate along with quercetin-4'-glucoside, quercetin and quercetin hydrate. The metabolites of quercetin in the rat stomach were thought to be produced by the action of microorganisms present in the rat stomach. After $1 \mathrm{~h}, 25 \%$ of the ingested radioactivity was traveled from the stomach to the duodenum (contained $8 \%$ of the intake) and to jejunum (contained $25 \%$ of the intake) and finally to ileum (contained $26 \%$ of the intake). The glucuronidation first occurred in the duodenum containing quercetin-4'-glucoside, quercetin, quercetin glucuronide, quercetin diglucuronide and 
<smiles>O=C(O)Cc1cc(O)cc(O)c1</smiles><smiles>O=C(O)Cc1cc(O)c(O)c(O)c1</smiles>

Figure 6. Examples of myricetin metabolites (a, 3,5-dihydroxyphenylacetic acid; b, 3,4,5-trihydroxyphenylacetic acid) (Lin et al., 2012; Vissiennon et al., 2012).

methyl quercetin diglucuronide. The ingested quercetin substrate travel across the GI tract for 1-6 h, producing various glucuronides, methylated and sulfated derivatives of quercetin, however, only trace amounts were excreted in the urine. The results indicated that between 6 and $12 \mathrm{~h}$, the $\left[2-{ }^{14} \mathrm{C}\right]$ quercetin- 4 '-glucoside intake showed a decline of the corresponding radioactivity in the GI tract and an increase in the amount of radioactivity in the feces $(9.4 \%)$ and urine $(52 \%)$. When the substrate reaches the colon, various glucuronide conjugates formed in the small intestine were hydrolyzed by the colonic microflora and released in the form of aglycones.

As reported in various studies, ring fission produced $\left[{ }^{14} \mathrm{C}\right] 3,4-$ dihydroxyphenylacetic acid which was further dehydroxylated to form 3-hydroxyphenylacetic acid and traces of 3,4-dihydroxybenzoic acid. The 3-hydroxyphenylacetic acid produced further underwent $\alpha$-oxidation and dehydroxylation and was converted to hippuric acid and finally to benzoic acid. The study revealed presence of various phenolic acids, namely 3-hydroxyphenylacetic acid, hippuric acid, and benzoic acid in urine and feces except for benzoic acid. This study indicated that identification of phenolic acid in the feeding studies using unlabelled substrates with subjectto-subject variations in the colonic microflora and various routes independent of dietary flavonoid intake to produce phenolic acids was very difficult. Hippuric acid, for instance, could be derived from benzoic acid, quinic acid, tryptophan, tyrosine, and phenylalanine. The authors noted that this was the first time hippuric acid was demonstrated as being a degradation product of quercetin due to the use of radiolabeled material.

\subsubsection{Metabolism of kaempferol and myricetin}

Flavonols such as kaempferol, quercetin and myricetin differ only slightly in their structures with the number of hydroxyl groups at the $\mathrm{B}$ ring. These compounds share identical degradation pathways and after cleavage of the C-ring are converted to $p$-hydroxyphenylacetic acid ( $p$-HPAA), 3,4-dihydroxyphenylacetic acid (DOPAC) with subsequent degradation to $m$-hydroxyphenylacetic acid ( $m$ HPAA) and 3,4,5-trihydroxyphenylacetic acid with subsequent degradation to 3,5-dihydroxyphenylacetic acid, respectively (Vissiennon et al., 2012). Serra et al. (2012) studied kaempferol metabolism by rat colonic microflora and reported that the primary metabolite were phenylacetic acid and $p$-hydroxybenzoic acid at 15 and $4.2 \mathrm{pM}$ concentrations, respectively, after $48 \mathrm{~h}$ of incubation. The results also revealed that kaempferol-rutinoside substrate was completely metabolized after $24 \mathrm{~h}$ of incubation. Similar results were obtained during fermentation of myricetin; phenylacetic acid was obtained as the primary metabolite at 78 pM concentration after $48 \mathrm{~h}$ of incubation. However, the overall metabolism of myricetin was poor in colonic fermentation. Examples of myricetin metabolites are shown in Figure 6 (Smith and Griffiths, 1970; Vissiennon et al., 2012).

\subsubsection{Metabolism of flavanones}

\subsubsection{Metabolism of naringenin}

Naringenin, which can be found in grapefruit, consists of flavanone structure with three hydroxyl groups $\left(5,7,4^{\prime}-\mathrm{OH}\right)$ (Felgines et al., 2000). Orrego-Lagarón et al. (2015) studied the permeability of naringenin in stomach, small intestine and colon as well as the metabolism, microbiota absorption and deposition of naringenin. The results indicated that the stomach and small intestine metabolites were dominated by the formation of naringeninglucuronide (NAR-GLU) and naringenin sulfate (NAR-SULF). The colonic metabolites consisted of naringenin sulfate (NARSULF), 4-hydroxyhippuric acid, hippuric acid, and naringeninglucuronide (NAR-GLU). The phase II metabolites in the bile and plasma from colon perfusion experiments revealed the presence of naringenin-glucuronide (NAR-GLU), 3-(4-hydroxyphenyl) propionic acid, 4-hydroxyhippuric acid, hippuric acid, 3-phenylpropionic acid, and (4-hydroxyphenyl)acetic acid in the control samples except naringenin sulfate (NAR-SULF) which was undetectable. The results were expressed in the areas under the lumen concentration curve (AUC) and the total conjugates excreted were as: small intestine $>$ colon $>$ stomach. The study concluded that naringenin was a highly permeable compound and was absorbed mainly in the small intestine by passive diffusive mechanism. However, low oral bioavailability of naringenin was attributed to high intestinal first-pass effect and minor hepatic metabolism. The high intestinal first pass metabolism of naringenin led to a high level of phase II metabolites, mainly naringenin- $O$-sulfate in the intestinal lumen. The health effects of naringenin were more related to the high level of bioactive metabolites formed than to its bioavailability.

Serra et al. (2012) studied naringenin metabolism by rat colonic microflora. The results indicated intense metabolism of naringenin and its complete degradation by colonic microflora at $48 \mathrm{~h}$. During metabolism of naringenin, metabolites such as phenylacetic acid and $p$-hydroxyphenyl acetic acid were present in the system from 0 to $48 \mathrm{~h}$. The compound 3-(4-hydroxyphenyl) propionic acid was produced in the last phase of metabolism at 24 and $48 \mathrm{~h}$. The results from this study were different from others reported in the literature (Orrego-Lagarón et al., 2016; Rechner et al., 2004).

\subsubsection{Hesperetin}

Hesperetin has only one hydroxyl group in its B ring and is frequently found in citrus fruits (Shahidi and Ambigaipalan, 2015). In a different study, plasma and urinary metabolites and catabolites derived from orange juice polyphenols were identified. The main components were hesperetin-7-O-rutinoside (hesperidin), naringenin-7-O-rutinoside (narirutin), apigenin-6,8-C-diglucoside (vicenin-2), ferulic acid-4'-O-glucoside coumaric acid-4'-Oglucoside and 4'-O-methylnaringenin-7-O-rutinoside (didymin). Small quantities of other flavanones, such as hesperetin-7-Orutinoside-3'-O-glucoside and eriodictyol-7- $O$-rutinoside (eriocitrin) were also present along with a sinapic acid- $O$-hexoside (Pereira-Caro et al., 2016).

The hesperetin derivatives in orange juice dominated with $63 \%$ of the total polyphenol content, followed by $19 \%$ naringenin derivatives and $1 \%$ other polyphenols. The human intervention trial after orange consumption was conducted on 12 men who were on a polyphenol-free diet for two days prior to the start of the trails. The 
urine and plasma samples obtained from volunteers were analyzed for their polyphenol contents and catabolites. The results quantified 10 hesperetin, 7 naringenin, and 2 eriodictyol metabolites in urine after consumption of orange juice, while 8 hesperetin and 3 naringenin metabolites were detected in plasma. In the literature, it was suggested that not all flavanone aglycones released in the distal GI tract were absorbed. Some of these metabolites undergo microbial mediated metabolism with ring fission and yield a family of low molecular weight phenolic catabolites that are absorbed in the liver and kidney before entering systemic circulation. The result from this study also emphasized their involvement in the overall bioavailability of orange juice polyphenols as well as subsequent hepatic conversions that led to hippuric acid and its hydroxylated analogues (Pereira-Caro et al., 2016).

\subsubsection{Metabolism of flavone}

\subsubsection{Luteolin}

Luteolin $\left(3^{\prime}, 4^{\prime}, 5,7\right.$-tetrahydroxyflavone) exists in vegetables such as celery, parsley, broccoli, and cabbages (Lin et al., 2008). Braune et al. (2001) studied the degradation of flavonol quercetin and flavone luteolin by an anaerobic bacteria Eubacterium ramulus. The degradation experiments were carried out in resting cell suspensions. Individual cell samples were centrifuged, and the pellets were lyophilized and dissolved in methanol. The metabolites were detected using HPLC. The results indicated the formation of eriodictyol as an intermediate in the resting-cell fermentation. The study proposed a pathway in which the degradation starts with the reduction of double bond in the 2,3-position prior to C-ring fission leading to the formation of eriodictyol. The eriodictyol was then reduced into two intermediate chalcones. The final product of luteolin degradation was 3-(3,4-dihydroxyphenyl)propionic acid.

Shimoi et al. (2002) studied the intestinal absorption of luteolin and luteolin $7-O-\beta$-glucoside in rats by HPLC. The absorption study of rat indicated that luteolin was converted to glucuronides during their passage through intestinal mucosa and luteolin 7-O- $\beta$ glucoside was poorly absorbed by itself and luteolin glucuronides were detected after intestinal absorption. Also, the authors reported that plasma of the rats contained free luteolin, glucuronide or sulfate-conjugates of unchanged luteolin and o-methyl-luteolin. This result suggests that luteolin can escape intestinal conjugation and the hepatic sulfation or methylation.

Serra et al. (2012) studied colonic metabolism of luteolin using rat microflora with a starting concentration of $32 \mathrm{pM}$. The results indicated that luteolin was poorly metabolized and almost $5 \mathrm{pM}$ of luteolin was detected in the fermentation after $48 \mathrm{~h}$ incubation. The only metabolic product achieved was 3-(2,4-dihydroxyphenyl)propionic acid at very low concentration of $0.64 \mathrm{pM}$.

\subsubsection{Metabolism of flavanols}

\subsubsection{Catechin and epicatechin}

Catechin and epicatechin belong to flavan-3-ol, found in cocoa, tea extract, and lentils (Kwik-Uribe and Bektash, 2008; Zhong et al., 2012; Yeo and Shahidi, 2017).

The absorption and metabolic study of catechin and epicatechin indicated that it was rapidly absorbed in the upper portion of small intestine. After absorption, these monomers undergo phase
II metabolism in the intestine and liver to form glucuronidated, sulfated, and/or methylated conjugates and these metabolites are transported into blood via portal vein and absorbed in tissues. Major conjugates of epicatechin in human plasma, bile, and urine were (-)-epicatechin $3{ }^{\prime}-O$-sulfonate and (-)-epicatechin $3{ }^{\prime}-O-\beta$ glucuronide (Spencer et al., 2001).

Various studies suggest that in vivo metabolism of catechin and epicatechin produced 3-(3-hydroxyphenyl)propionic acid in human urine and feces. In addition, in vivo metabolism of catechin and epicatechin also produced 5- $\delta$-(3,4-dihydroxyphenyl)$\gamma$-valerolactone, 5- $\delta$-(3-hydroxyphenyl)- $\gamma$-valerolactone, 3-(3-hydroxyphenyl)propionic acid, 3-hydroxyhippuric acid in rat urine. In vitro metabolism studies of catechin and epicatechin reported production of 3-(3,4-hihydroxyphenyl)propionic acid, 3-(3-hydroxyphenyl)propionic acid, 5-(3,4-dihydroxyphenyl)valeric acid, and 3-phenylpropionic acid in human fecel microbiota (Aura et al., 2008; Das, 1971).

The metabolic pathways of various flavanols such as catechin and epicatechin were studied by an in vitro colonic fermenation model using rat colonic microflora for $48 \mathrm{~h}$ (Serra et al., 2011). The results showed that there were significant differences in the quantity of metabolites formed when two stereoisomers catechin and epicatechin were fermented. The main metabolite formed during the catechin fermenation was phenylacetic acid at $24 \mathrm{pM}$ after $48 \mathrm{~h}$ incubation, however, epicatechin fermentation produced 58 $\mathrm{pM}$ after $48 \mathrm{~h}$ incubation. The main metabolic product of epicatechin was 5-(hydroxyphenyl)- $\gamma$-valerolactone at $142 \mathrm{pM}$ after 48 $\mathrm{h}$ incubation, whereas the same metabolite was produced during catechin fermentation at $18 \mathrm{pM}$ after $48 \mathrm{~h}$. During the whole fermentation process both the isomers were metabolized however, catechin catabolism was not complete and quantified at $1.2 \mathrm{pM}$ after $48 \mathrm{~h}$ incubation. Whereas, there was complete catabolism of epicatechin at $48 \mathrm{~h}$ of incubation. In the same manner, different position isomers of hydroxyphenylacetic acid was also obtained during the fermentation of catechin and epicatechin. Catechin fermentation produced 2 and 4-hydroxyphenylacetic acid, however, epicatechin fermentation produced 3-hydroxyphenylacetic acid. These hydroxyphenylacetic acids were suggested be dehydroxylated to phenylacetic acid, in both the catechin and epicatechin fermentation mediums. The examples of catechin metabolites are shown in Figure 7.

Roowi et al. (2010) studied the colonic degradation of green tea flavanols and urinary excretion of catabolites by humans. Theresults indicated that after incubation of epicatechin in fecal slurries, the predominant degradation products (-)-5-(3',4'-dihydroxyphenyl)$\gamma$-valerolactone, 5-(3,4-dihydroxyphenyl)- $\gamma$-valeric acid, and 3-(3-hydroxyphenyl)propionic acid. In the urinary excretion, 3-(3-hydroxyphenyl)- 3-hydroxypropionic acid was detected. The result was slightly different from the study carried out by Serra et al. (2011), in which different catabolites were identified. It is evident from this study, that most of the metabolism of epicatechin is carried out by colonic fermentation where they are degraded into various phenolic acid catabolites after being absorbed and passing through the circulatory system, are excreted in urine in quantities corresponding to approximately $40 \%$ of flavanol intake. The proposed metabolic pathway of epicatechin is shown in Figure 8.

\subsubsection{Epigallocatechin gallate (EGCG) and epicatehcin gallate (ECG)}

EGCG and ECG are another example of flavan-3-ol family and these have ester bond at $3-\mathrm{OH}$ with gallic acid. Among tea cat- 
<smiles>O=C(O)Cc1ccccc1</smiles>

Phenylacetic acid<smiles>Oc1cccc(C[C@@H](O)Cc2c(O)cc(O)cc2O)c1</smiles>

\section{Diarylpropan-2-ol}

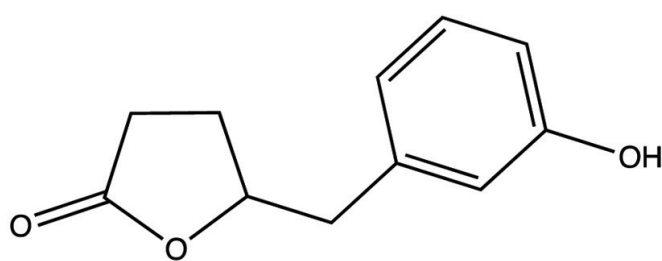

5-(Hydroxyphenyl)- $\gamma$-valerolactone<smiles>O=C(O)CCc1ccc(O)c(O)c1</smiles>

3-(3,4-Dihydroxyphenyl)propionic acid

Figure 7. Examples of catechin metabolites (Serra et al., 2011).

echin, epigallocatechin gallate is most abundent (Zhong et al., 2012).

The fermentation of epigallocatechin gallate (EGCG) and epicatechin gallate (ECG) was carried out with an initial concentration of 8.5 and $3.7 \mathrm{pM}$ at $0 \mathrm{~h}$, respectively. The $p$-hydroxybenzoic, protocatechuic, phenylacetic, 2-hydroxyphenylacetic and 4-hydroxyphenylacetic acids were the common metabolites obtained from the metabolism of epigallocatechin gallate (EGCG) and epicatechin gallate (ECG). The presence of epicatechin was found throughout the incubation of EGCG for $48 \mathrm{~h}$, however, the epicatechin reached its maximum of $8.2 \mathrm{pM}$ during ECG fermenation at $4 \mathrm{~h}$ and then was not detected after $24 \mathrm{~h}$ incubation. The major metabolites of EGCG fermentation were phenylacetic acid and 4-hydroxyphenylacetic acid at 35 and $39 \mathrm{pM}$ at $48 \mathrm{~h}$, respectively. The major metabolites during ECG fermentation were phenylacetic acid and 4-hydroxyphenylacetic acid at 25 and $19 \mathrm{pM}$ at $48 \mathrm{~h}$, respectively. Similar to the metabolism of catechin and epicatechin, 5-(3,4-dihydroxyphenyl)- $\gamma$-valerolactone and 5-(hydroxyphenyl)$\gamma$-valerolactone were quantified during colonic ferementation of EGCG and EGC respectively, but at a lower concentration (Serra et al., 2011).

The microbial metabolism of ECG usually starts by rapid cleavage of the gallic acid ester moiety by microbial esterases giving two products gallic acid and epicatechin. The gallic acid produced is further decarboxylated into pyrogallol. The c-ring of the epicatechin is subsequently opened producing $1-\left(3^{\prime}, 4^{\prime}\right.$-dihydroxyphenyl)-3$\left(2^{\prime \prime}, 4^{\prime \prime}, 6^{\prime \prime}\right.$-trihydroxyphenyl)-propan-2-ol, which is later converted into 5-(3',4'-dihydroxyphenyl)- $\gamma$-valerolactone. However, in the case of epigallocatechin gallate, 5-(3',4',5'-trihydroxyphenyl)$\gamma$-valerolactone is produced as a product. The valerolactone substrates are later converted into 5-(3',4'-dihydroxyphenyl) valeric acid and/or 4-hydroxy-5-(3',4'- dihydroxyphenyl) valeric acid.
Subsequent metabolic activities such as $\alpha$ and $\beta$-oxidation, methylation and dehydroxylation reaction leads to the formation of various hydroxyphenyl propionic and hydroxybenzoic acids.

\subsubsection{Metabolism of isoflavones}

Isoflavones are a group of phenolic compounds which are mainly present in the legumes and soy-based food. Isoflavones are the most potent phytoestrogens found in plants as they bind to the estrogen receptor and affect the conformational dynamics in ways distinct from the endogenous ligand estrogen. Therefore, isoflavones are classified as selective estrogen receptor ligand (Boué et al., 2003). The metabolism of isoflavones such as daidzein and genistein undergo bioactivation to the isoflavandiol equol. The biosynthetic pathway of equol production starts with the achiral substrate daidzein and all subsequent reaction steps proceed stereo specifically with chiral intermediates. The aglycone of daidzein is metabolized in liver and transformed into more water-soluble metabolites via glucuronidation and sulfation. The intestinal sequential reduction or hydrogenation reaction results in the formation of the following metabolites dihydrodaidzein (DHD, 4',7-dihydroxyisoflavone), tetrahydrodaidzein (THD, 4',7- dihydroxyisoflavan-4-ol) and equol (4',7-dihydroxyisoflavan). An alternative metabolic route produces $O$-desmethylangolensin (O-DMA) via aromatic C-ring cleavage. It is also reported that $O$-desmethylangolensin can further partially metabolized into resorcinol and 2-(4-hydroxyphenyl) propionic acid. The metabolic cycle of daidzein is shown in Figure 9 (Braune and Blaut, 2011, 2016; Frankenfeld, 2012; Lee et al., 2017; Stevens and Maier, 2016).

Lee et al. (2017) demonstrated the biosynthesis of (-)-5-hydroxyequol and 5-hydroxydehydroequol from soy isoflavone called 
<smiles>Oc1cc(O)c2c(c1)O[C@H](c1ccc(O)c(O)c1)[C@H](O)C2</smiles>

Epicatechin<smiles>Oc1cc(O)c(CC(O)Cc2ccc(O)c(O)c2)c(O)c1</smiles>

1-(3',4')-dihydroxyphenyl-3-(2",4",6"-trihydroxy)propan-2-ol<smiles>O=C1CCC(Cc2ccc(O)c(O)c2)O1</smiles>

(-)-5-(3', 4'-dihydroxyphenyl)- $-\gamma$-valerolactone<smiles>O=C(O)CCCCc1ccc(O)c(O)c1</smiles>

5-(3,4-dihydroxyphenyl)- $\nu$-valeric acid<smiles>[CH]1C=C1</smiles><smiles>O=C(O)CCc1cccc(O)c1</smiles>

3-(3-hydroxyphenyl)propionicacid<smiles>O=C(O)CC(O)c1cccc(O)c1</smiles>

3-(3-hydroxyphenyl)-3-hydroxypropionicacid

Figure 8. Proposed metabolic pathway of epicatechin (adopted from Roowi et al., 2010).

genistein using microbial whole cell bioconversion. This study indicated that like reduction of daidzein to (S)-equol there was no direct evidence that reduction of genistein would yield 5-hydroxyequol. The three reductase enzymes namely daidzein reductase (DZNR), dihydrodaidzein reductase (DHDR), and tetrahydrodaidzein reductase (THDR) did not convert genistein to 5-hydroxyequol. Only the first reaction step of conversion of genistein to dihydrogenistein using DZNR and other steps were reported to be unclear in the literature. Therefore, the researchers from this study used recombinant $E$. coli cells expressing three reductases DZNR, DHDR, and THDR as well as another dihydrodaidzein racemase
(DDRC) to biosynthesize genistein into 5-hydroxy-equol. The study produced a novel isoflavone called 5-hydroxy-dehydroequol as a by-product after DHDR reaction with an unknown dehydration mechanism.

\subsubsection{Metabolism of proanthocyanidins/condensed tannins}

Proanthocyanidins are polymeric phenolic compounds that 2 or more flavan-3-ols are linked together (Chandrasekara and Shahidi, 2010). Proanthocyanidins such as dimers A (C2 $\rightarrow$ O7) and dimers $\mathrm{B}$ (linked by $\mathrm{C} 4 \rightarrow \mathrm{C} 8$ and/or $\mathrm{C} 4 \rightarrow \mathrm{C} 6$ bonds) the bioavailability is influenced by their degree of polymerization. The degree of polymerization of proanthocyanidins range in the form of oligomers (2-4 monomers), polymers ( $>4$ monomers) and high-molecular-weight polymers ( $>10$ monomers). The absorption rate of proanthocyanidin dimers is about $5-10 \%$ of that of epicatechin. The timer and tetramer proanthocyanidin have lower absorption rate than dimers and absorbed fraction of trimers and tetramers undergo only limited phase II metabolism in the intestine and liver compared to epicatechin. Most of the proanthocyanidins with higher degree of polymerization are not absorbed in the intestine and directly reach colon in the intact form. The colon microflora break proanthocyanidins into various phenylvalerolactones and phenolic acids (Gu et al., 2003; Ou and Gu, 2014).

The metabolic pathways of various proanthocyanindin dimer B2 (epicatechin- $(4 \beta \rightarrow 8)$-epicatechin) was studied by an in vitro fermenation model using rat colonic microflora for $48 \mathrm{~h}$ (Serra et al., 2011). Colonic fermentation of proanthocyanidin dimer (epicatechin- $(4 \beta \rightarrow 8)$-epicatechin) using rat colonic microflora revealed that the dimers were rapidly hydrolyzed into epicatechin monomers. The study started with $0.03 \mathrm{pM}$ at $0 \mathrm{~h}$ and the dimer was completely hydrolyzed reaching $0.01 \mathrm{pM}$ at $4 \mathrm{~h}$ and $0 \mathrm{pM}$ at $24 \mathrm{~h}$ in the fermentation medium. Immediately after the incubation, epicatechin monomers were seen in the fermentation medium at $0.14 \mathrm{pM}$ after $0 \mathrm{~h}$ and increased to $1.07 \mathrm{pM}$ after $2 \mathrm{~h}$ of incubation, however, epicatechin monomers disappeared after $4 \mathrm{~h}$ in the fermenation medium. Phenylacetic acid and 4-hydroxyphenylacetic acid were the main metabolite at 29 and $27 \mathrm{pM}$ after $48 \mathrm{~h}$ incubation, respectively. No valerolactone and its precursors were seen in the fermentation medium of dimer B2, in contrast to epicatechin fermentation. This result can be attributed to two reasons, the first being a lower fermenation of dimer form of epicatechin than epicatechin monomer and the precursor to 5-(hydroxyphenyl)- $\gamma$ valerolactone, the diaryl-propan-2-ol was not formed within $48 \mathrm{~h}$ of fermentation. The second reason being limited metabolism of procyanidin dimer focused mainly on the upper epicatechin unit of dimer.

Sánchez-Patán et al. (2011) studied the biological relevance of a metabolite 5-(3',4'-dihydroxyphenyl)- $\gamma$-valerolactone usually formed during the catabolism of dietary flavanols such as dimeric proanthocyanidins by fecal fermentation collected from three healthy volunteers. The results from this study indicated large individual variations in the formation of 5-(3',4',5'-trihydroxyphenyl)$\gamma$-valerolactone between volunterrs with concentrations ranging between 3.31 and $77.54 \mu \mathrm{M}$ at $10 \mathrm{~h}$ of fermentation.

The metabolic degradation of proanthocyanidins by gut microflora is a complicated process involving various reactions such as hydrolysis, hydrogenation, $\alpha$ - and $\beta$-oxidation, dehydroxylation, demethoxylation, and decarboxylation resulting in the formation of various phenolic and aromatic catabolites. The catabolism of monomeric flavanols and dimeric proanthocyanidins are speculated by many researchers and different metbolic pathways are reported in the literature. The first step of microbial degradation 


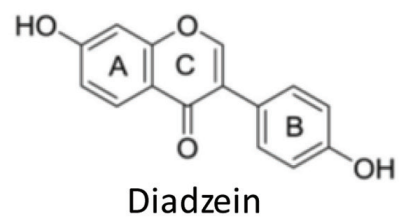

Daidzein

reductase

(DZNR)<smiles>O=C1c2ccc(O)cc2OCC1c1ccc(O)cc1</smiles>

Dihydrodaidzein (DAD)

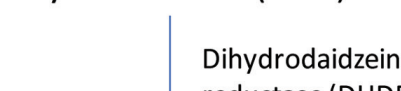

reductase (DHDR)<smiles>Oc1ccc([C@@H]2COc3cc(O)ccc3[C@@H]2O)cc1</smiles>

(3R,4S)-Tetrahydrodaidzein (THD)

Tetrahydrodaidzein

reductase (THDR)<smiles>Oc1ccc([C@H]2COc3cc(O)ccc3C2)cc1</smiles>

(3S)-Equol

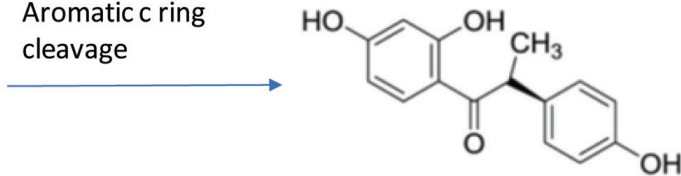

O-desmethylangolensin (O-DMA)<smiles>CCCCCCCCCCCCC(=O)C(O)c1ccc(O)cc1</smiles>

Resorcinol
2-(4-Hydroxyphenyl)propionic acid

Figure 9. Metabolic pathway of diadzein (Braune and Blaut, 2011, 2016; Frankenfeld, 2012; Lee et al., 2017; Stevens and Maier, 2016).

of dimeric procyanidin involves reductive cleavage of the heterocyclic $\mathrm{C}$ ring, resulting in the formation of diphenylpropan2-ols. This step is followed by the breakdown of the A ring and further lactonization process to afford various phenylvalerolactones derivatives. The 5-(3',4'-dihydroxyphenyl)- $\gamma$-valerolactone is a metabolized product of epicatechin, whereas trihydroxylated derivative, 5-(3',4',5'-trihydroxyphenyl)- $\gamma$-valerolactone is a metabolized product of epigallocatechins which is then further metabolized into $3^{\prime}, 4^{\prime}$ or $3^{\prime}, 5^{\prime}$-dihydroxylated forms. The metabolism of these flavanols also includes the production of phenylvalerolactones derivatives exclusively from the catabolism of the top unit of the procyanidin molecule. The metabolic pathway also has different pathways for the same molecule and interconversion of 5-(3',4'-dihydroxyphenyl)- $\gamma$-valerolactone with an open form 4-hydroxy-5-(3,4-dihydroxyphenyl)-varic acid is also reported. The valeric acid derviatives such as 5-( $3^{\prime}, 4^{\prime}$-dihydroxyphenyl)valeric acid and 4-hydroxy-5-(3'-hydroxyphenyl)-valeric acid undergo dehydroxylation to yield mono- and dihydroxylpropionic acid derviatives. This propionic acid derivatives are further metabolized via $\alpha$ and $\beta$-oxidation to produce mono- and diacetic and benzoic acid derviatives (Appeldoorn et al., 2009; Monagas et al., 2010; Sánchez-Patán et al., 2011; Stoupi et al., 2010).

\subsubsection{Metabolism of hydrolyzable tannins}

The major dietary source of ellagitannins in the western diet originates from red fruits such as strawberries, raspberries and blackberries. There are no definitive studies on the absorption and metabolism of ellagitannins in humans (Clifford and Scalbert 2000). González-Barrio et al. (2010) studied the bioavailability of ellagitannins following consumption of raspberries (300 g) by healthy humans and subjects with an ileostomy. Post consumption, the plasma and urine samples were collected and analyzed using HPLC-MDA-MS/MS for up to $48 \mathrm{~h}$. The results from this study indicated that ellagic acid $(19 \mu \mathrm{mol})$ and sanguiin H-6 $(23 \mu \mathrm{mol})$ was recovered over the $48 \mathrm{~h}$ period. The amount of ellagic acid and sanguiin H-6 present in the ileal fluid was 241 and $23 \%$, respectively, of the $7.9 \mu \mathrm{mol}$ intake. Ellagic acid- $O$-pentoside, lambertianin $\mathrm{C}$ and sanguiin $\mathrm{H}-10$ were not detected in the ileal fluid. The recovery of ellagic acid and metabolites from urine collected from volunteers with an intact colon and subjects with an ileostomy was also analyzed. Volunteers with an intact colon had 7.9 and $5.2 \mathrm{nmol}$ ellagic acid and ellagic acid- $O$-glucuronide, respectively, in their urine samples compared to 26 and $7.8 \mathrm{nmol}$, respectively, 
in the urine samples of ileostomy volunteers. The study also indicated that consumption of ellagitannins food resulted in the formation of urolithin metabolites produced in the colon by microflora mediated degradation in varying concentrations. There were large subjective variations reported in this study and the spectrum of urolithins also varied.

Bioavailability of ellagic acid in human plasma after consumption of ellagitannins from pomegranate juice $(180 \mathrm{~mL})$ containing ellagic acid (25 mg) and ellagitannins (318 $\mathrm{mg}$, as punicalagins) was studied and the results indicated the presence of ellagic acid in the range of $16.2-31.9 \mathrm{ng} / \mathrm{mL}$ concentrations between 0 and 6 $\mathrm{h}$ time intervals. The study also revealed that no ellagitannin was found in the intact form in the plasma (Seeram et al., 2004). However, various ellagic acid derived metabolites such as urolithin A, hydroxyurolithin A, urolithin A-glucuronide, urolithin A and dimethyl ellagic acid-glucuronide were found after pomegranate juice consumption by volunteers (Mertens-Talcott et al., 2006). Piwowarski et al. (2016) studied the differences in metabolism of ellagitannins by human gut microbiota in ex vivo cultures. In this study fifteen monomeric and dimeric ellagitannins such as (1) ellagic acid, (2) 1-O-galloyl-4,6-(S)-HHDP- $\beta$-D-glucose, (3) pedunculagin, (4) potentillin, (5) casuarictin, (6) coriariin B, (7) vescalagin, (8) castalagin, (9) stachyurin, (10) casuarinin, (11) stenophyllinin A, (12) stenophyllanin A, (13) salicarinin A, (14) gemin A, (15) agrimoniin, and (16) oenothein B were studied. The results indicated that differences existed in amounts of produced urolithins which indicated that the individual microbiota composition and type of ingested ellagitannins determine the rate of urolithin production. The results from this study also suggested that hexahydroxydiphenoyl (HHDP) present in the ellagitannin molecules provided HHDP groups as substrates for microbiota metabolism but also depending on their structure and microbiota metabolite types, influenced the growth and/or metabolism among the microbiota ecosystems in the urolithin metabolic pathway.

Espín et al. (2007) used Iberian pig as a model to study the metabolism of ellagitannin in humans. The pigs were fed on either a cereal fodder or acrons which are a rich source of ellagitannins. The plasma, urine, bile, lumen and intestinal tissues (jejunum and colon), feces, liver, kidney, heart, brain, lung, muscle, and subcutaneous fat tissue were analyzed after necropsy. The results from this analysis indicated that acron ellagitannins release ellagic acid (EA) in the jejunum which is then subjected to metabolism by intestinal microflora to sequentially yield tetrahydroxy-(urolithin D), trihydroxy-(urolithin $\mathrm{C}$ ), dihydroxy-(urolithin A), and monohydroxy-(urolithin B) dibenzopyran-6-one metabolites, which were absorbed preferentially when their lipophilicity increased. Also, 31 ellagitannin derived metabolites were detected including 25 urolithins and 6 ellagic acid derivatives. The analysis of bile revealed that 26 extensively conjugated metabolites such as glucuronides and methyl glucuronides of ellagic acid and particularly urolithin $\mathrm{A}, \mathrm{C}$, and $\mathrm{D}$ derivatives were found in bile samples, confirming a very active enterohepatic circulation. In addition, only urolithin A and $\mathrm{B}$ as well as dimethylellagic acid-glucuronide was detected in the peripheral plasma. This study also demonstrated that ellagic acid derivatives were found in bile and in urine and it was absent in the intestinal tissues indicating complete absorption in the stomach. Furthermore, only urolithin A was found in the feces and together with its glucuronide was the most abundant metabolite in urine.

Tomás-Barberán et al. (2014) studied the metabolism of ellagic acid by human gut microbiota via intervention studies. During this study, the volunteers did not take antibiotics 4 weeks before the trails and avoided ellagitannin containing food products. Three different intervention studies were carried out and urine and fae- cal samples were obtained and processed. In the first intervention study, volunteers consumed $30 \mathrm{~g}$ of walnuts, in the second group volunteers consumed 4 pomegranate extract capsules and in the third group volunteers consumed 2 pomegranate extract capsules. The volunteers from the intervention study produced urolithin A, isourolithin A and/or urolithin B. However, the production of urolithin may be dependent on the age, gender, body mass index (BMI) and of the amount or type of ellagitannin food source ingested.

\subsubsection{Metabolism of tyrosol and oleuropein}

Tyrosol and oleuropein, which are known for their various health benefits such as antiviral, antioxidant, and anti-inflammatory effects, are found in olive, olive tree and olive oil (Sun et al., 2017; Sun et al., 2018). Faecal microbial metabolism of olive oil phenolic compounds was studied by an in vitro model using human faecal microbiota (Mosele et al., 2014). To determine the metabolism of olive oil four phenolic standards tyrosol, hydroxytyrosol, hydroxytyrosol acetate and oleuropein were selected in this study. The phenolic compounds were subjected to in vitro colonic fermentation for $48 \mathrm{~h}$. The results so obtained indicated that tyrosol metabolism produced only 2-(4'-hydroxyphenyl)acetic acid while hydroxytyrosol produced various metabolites including phenylacetic acid, 2-(4'-hydroxyphenyl)acetic acid and 2-(3',4'-dihydroxyphenyl)acetic acid. The colonic fermentation of hydroxytyrosol acetate produced hydroxytyrosol, phenylacetic acid, 2-(4'-hydroxyphenyl)acetic acid, 2-(3',4'-dihydroxyphenyl)acetic acid and 3(4'-hydroxyphenyl)propionic acid. Meanwhile, the colonic fermentation of oleuropein produced hydroxytyrosol, hydroxytyrosolacetate, elenolic acid and oleuropein aglycone. Based on these results, metabolic pathways of the proposed routes for the colonic metabolism of tyrosol, hydroxytyrosol and hydroxytyrosol acetate and oleuropein aglycone is shown in Figures 10 and 11. The metabolism of tyrosol, hydroxytyrosol and hydroxytyrosol acetate undergoes various processes including oxidation of primary molecules into hydroxylated phenylacetic acids in the presence of alcohol dehydrogenase and aldehyde dehydrogenase, dehydroxylation, de-esterification, hydrolysis of acetate molecule, carboxylation and $\alpha$-oxidation. The final metabolites of tyrosol, hydroxytyrosol and hydroxytyrosol acetate including 4-hydroxybenzoic acid and 1,2-dihydroxybenzene were not detected in the fermentation medium after $48 \mathrm{~h}$, thus suggesting that dehydroxylation is the preferential catabolic route of microorganisms present in human faeces. The oleuropein glucoside undergoes rapid deglycosylation to produce oleuropein aglycone followed by various hydrolysis processes by microbial esterase activity to elenolic acid, 3-(3',4'-dihydroxyphenyl)propionic acid, hydroxytyrosol acetate and hydroxytyrosol. These compounds undergo similar metabolic processes to various phenolic acids (Mosele et al., 2014).

Mateos et al. (2005) studied the metabolism of olive oil phenols such as hydroxytyrosol, tyrosol, and hydroxytyrosyl acetate by human hepatoma HepG 2 cells for 2 and $18 \mathrm{~h}$ incubation. The incubated samples were hydrolyzed using $\beta$-glucuronidase and sulfatase and analyzed by LC-MS. Standard solutions of olive oil phenols were enzymatically conjugated in vitro using pure enzymes such as UDP-glucuronosyltransferase (UGT) from rat liver for glucuronidation, cytosolic sulfotransferases from rat liver for sulfation and catechol-O-methyltransferase (COMT) and S-adenosyl-L-methionine (SAM) for methylation. The main metabolites during this study were glucuronides, methyl glucuronides, methyl conjugates and homovanillic acid. However, no sulfated conjugates were reported in this study. It has also been reported that glucuronidation 


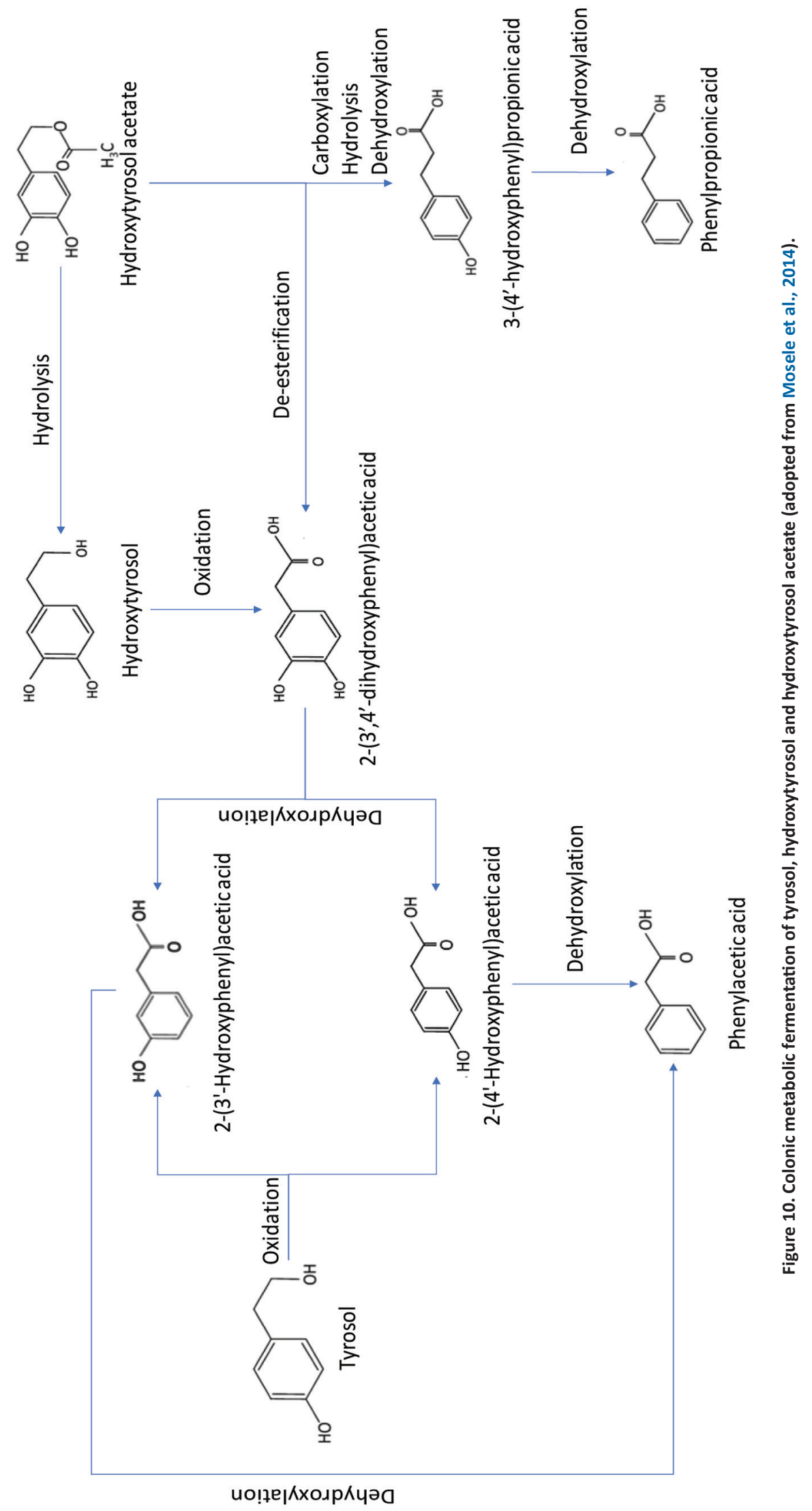




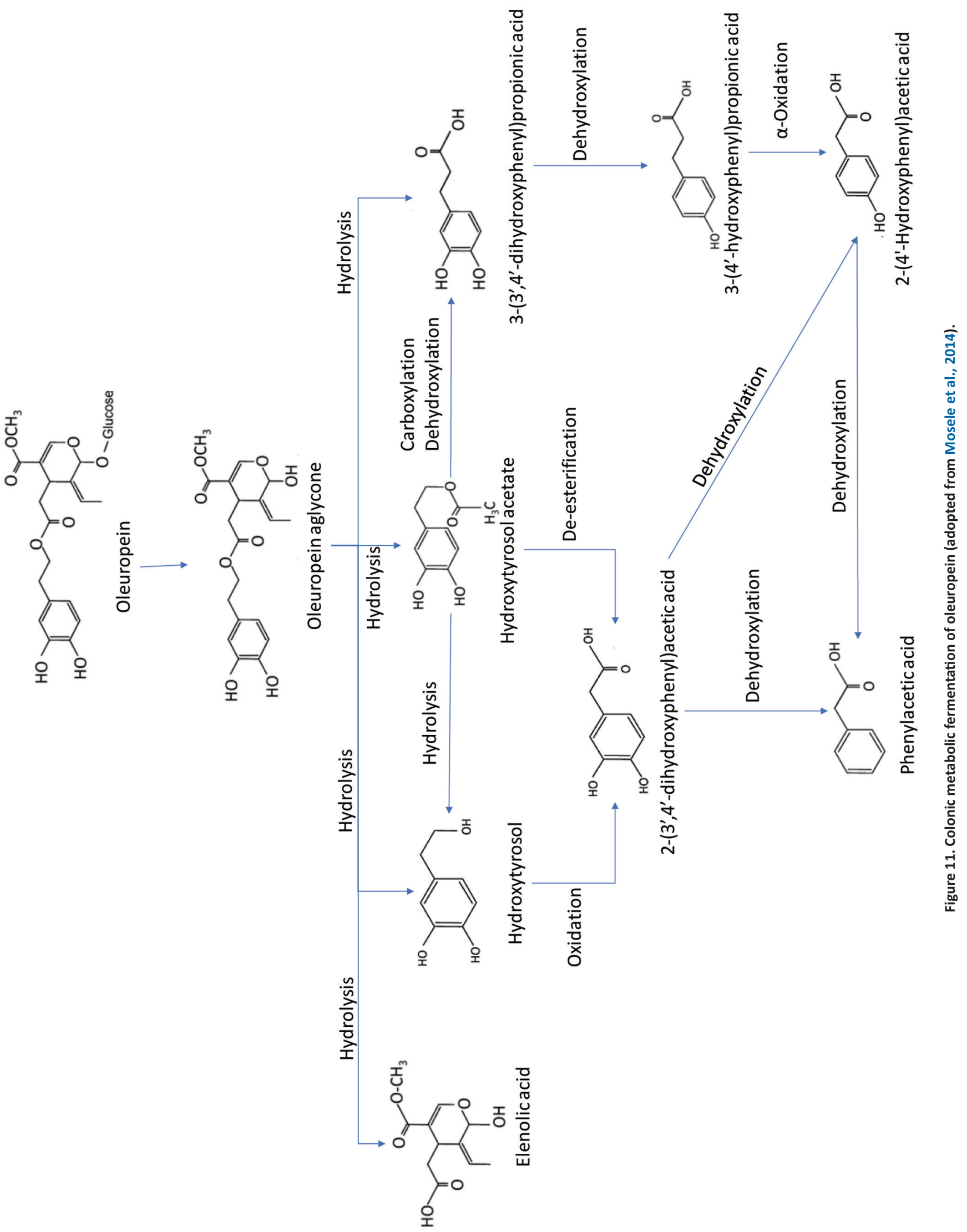


dominates metabolic reactions over methylation after $18 \mathrm{~h}$ incubation. Hydroxytyrosol undergoes both glucuronidation and methylation, whereas lacking an ortho-diphenolic structure could not be methylated by COMT and the only metabolite formed from tyrosol was glucuronides.

Corona et al. (2006) studied the absorption, metabolism and microflora-dependent transformation of olive oil polyphenols at different sites in the gastrointestinal tract. The results from this study indicated rapid increase in the presence of hydroxytyrosol and tyrosol in the small intestine, jejunum or ileum and demonstrated regular phase I/II metabolism pathways. The main metabolites identified in this study also indicated $O$-methylated derivatives of hydroxytyrosol, $O$-glucuronides of hydroxytyrosol and tyrosol, and a glutathionylated conjugate of hydroxytyrosol. Oleuropein was not metabolized in the small intestine and it was rapidly degraded by the colonic microflora resulting in the formation of hydroxytyrosol.

\subsubsection{Metabolism of stilbenes}

\subsubsection{Resveratrol}

Resveratrol (trans-3,5,4'-trihydroxystilbene) is a member of the stilbene family and a secondary metabolite produced by plants in response to biotic and abiotic stress. It is found in grapes, wine, peanuts and cranberries (Mei et al., 2015). Resveratrol is known to have antioxidant, anti-inflammatory, anti-cardiovascular, and anticancer activities (Oh and Shahidi, 2017; Oh et al., 2019; Wan et al. 2018). Bode et al. (2013) studied the in vivo and in vitro metabolism of trans-resveratrol by human gut microbiota. One part of the study was performed with feces samples from 7 healthy volunteers, and metabolite formation after fermentation was measured by liquid chromatography-ultraviolet/visible (UV/Vis)-mass spectrometry (MS)/MS detection. The second part of this study was carried out using controlled human intervention with 12 healthy volunteers. For this part, resveratrol was administered in the form of a colloid formulation and the dosage was standardized based on the trans-resveratrol content and was calculated as $0.5 \mathrm{mg}$ transresveratrol $/ \mathrm{kg}$ body weight. The results from the in vitro fermentation revealed that after $48 \mathrm{~h}$ of incubation, three metabolites were identified by LC-UV/Vis. One of the compounds initially identified as dihydroresveratrol was one of the major metabolic compounds found in 6 out 7 fecal samples as was as lunularin. In addition, a minor metabolite 3,4'-dihydroxy-trans-stilbene was identified in 1 out of 7 fecal samples. The in vivo metabolism of trans-resveratrol from the same study indicated that $19.6-41.1 \%$ of the trans-resveratrol was absorbed and recovered in the urine samples within 24 h. This indicates that a significant amount of trans-resveratrol was absorbed before transformation by intestinal bacteria. In addition, all 3 microbial metabolites found in the in vitro model were also detected in the urine samples with the 2 major metabolites being dihydroresveratrol and lunularin.

Resveratrol metabolites in various in vitro and in vivo studies on human, rat and mouse have been reported. These studies indicate that phase II metabolic enzymes and the gut microbiota paly a critical role in their transformation via glucuronidation and sulphation. The glucuronidation metabolites include transresveratrol-4'-O-glucuronide, rans-resveratrol-3-O-glucuronide, trans-resveratrol-diglucuronide and dihydroresveratrol-glucuronide. The sulfated metabolites included trans-resveratrol-3- $O$-sulfate, trans-resveratrol-4'-O-sulfate, cis-resveratrol-3-O-sulfate, transresveratrol-3,4'-disulfate, trans-resveratrol-glucuronide-sulfate, dihydroresveratrol-sulfate and dihydroresveratrol-glucuronide-sulfate (Andres-Lacueva et al., 2012; Herath et al., 2013; Mei et al., 2015; Springer and Moco, 2019; Yu et al., 2002).

\subsubsection{Metabolism of lignans}

Lignans are found in numerous foods such as oilseeds, whole grains, vegetables and fruits. It has been reported that secoisolariciresinol diglycoside and matairesinol are converted by intestinal bacteria after ingestion to mammalian lignans such as enterodiol and enterolactone (Niemeyer et al., 2003; Shahidi et al., 2019). Côrtes et al. (2008) studied the in vitro metabolism of flax lignans (hull and seed) by ruminal and faecal microbiota of dairy cows over $96 \mathrm{~h}$ with ruminal or faecal inoculum. The plant lignans present in flaxseeds and hulls were present at 9.2 and $32 \mathrm{nmol} / \mathrm{mg}$, respectively. The results from this study indicated that there was no difference in the net production of enterodiol between the hull and seed within the first $24 \mathrm{~h}$ of incubation. Also, the net production of enterolactone was significantly higher for flax products incubated with ruminl microorganisms, however, flax hulls produced more enterolactone than flaxseeds. The metabolism using faecal microbiota leads to the production of enterodiol.

Various studies have shown that intestinal bacteria are crucial for the metabolism of plant lignans and to produce enterolignans. During the process of enterolignan production, bacteria catalyze four sequential reactions, namely $O$-deglycosylation, $O$-demethylation, dehydrogenation and dehydroxylation and additional reduction steps for certain lignans. The reduction step in the lignan metabolism is carried out by Eggerthella lenta, Enterococcus faecalis and Eggerthella lenta intestinal microbiota. The deglycosylation step is due to the action of Bacteroides distasonis, Bacteroides fragilis, Bacteroides ovalus, Clostridium coclealum, Clostridium ramosum and Clostridium saccharogumia. The demethylation of lignans is carried out by Eubacterium limosum, Ruminococcus productus, Butyribacterium methylotrophicum and Eubacterium callanderi. The known intestinal bacteria responsible for dehydrogenation is Lactonifactor longoviformis. The dehydroxylation process is carried out using Clostridium scindens and Eggerthella lenta. There are several other unknown intestinal bacteria involved in the reduction and dehydroxylation processes during lignan metabolism as shown in Figure 12 (Clavel et al., 2006).

Gaya et al. (2016) studied the metabolism of various phytoestrogens of three different groups of isoflavones, lignans and ellagitannins by gut microorganisms and inter-individual differences were reported. The study demonstrated that both isoflavones and lignans are mostly found in the form of glycosides in food and after microbial deglycosylation, the phytoestrogens are metabolized by intestinal bacteria. The results from faecal fermentation studies indicated that out of 14 volunteers only one produced equol and others produced O-DMA. In addition, everyone produced urolithins and enterolactone from ellagitannin and lignans metabolism, respectively.

Mukker et al. (2014) studied the permeability and conjugative metabolism of flaxseed lignans by Caco-2 human intestinal cells. The study reported that secoisolariciresinol diglucoside present in the flaxseed undergoes metabolism to secoisolariciresinol, enterodiol and enterolactone in human GI tract. The lignans are present in the system as phase II enzyme conjugates. To further understand the oral absorption characteristics, an in-depth evaluation of two important processes of intestinal permeation and conjugation metabolism potential of lignans was deemed necessary. The results indicated that the permeability coefficients for secoisolariciresinol, enterodiol and enterolactone were $8.0 \pm 0.4,7.7 \pm 0.2$, and $13.7 \pm$ 

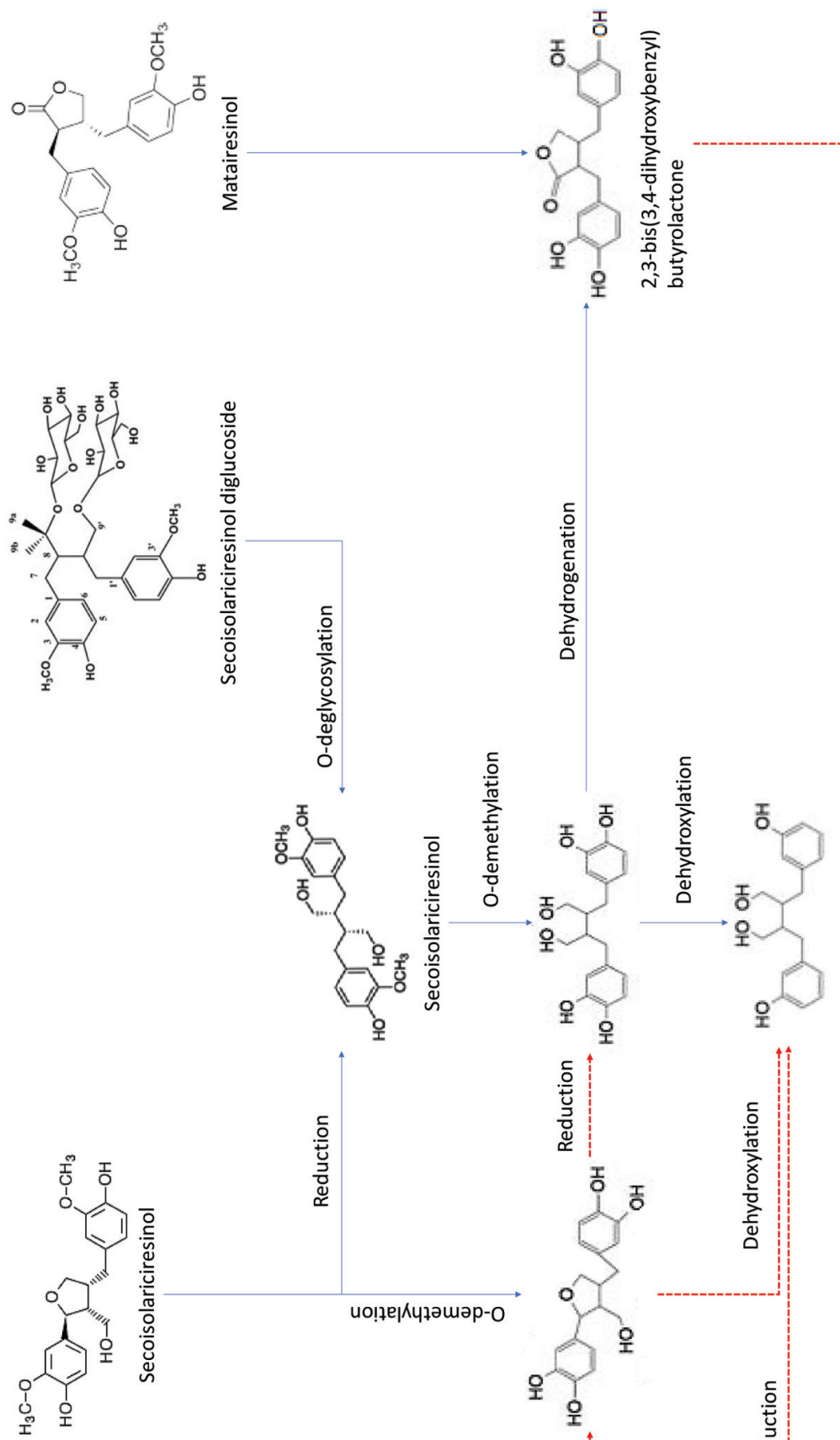

들
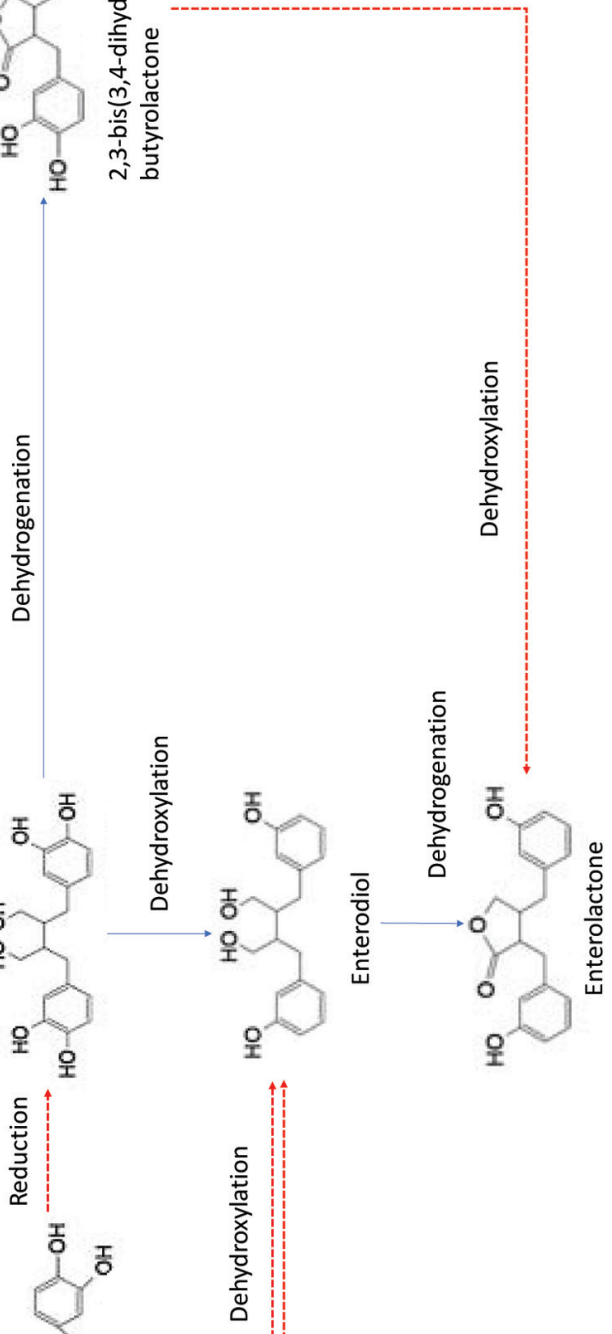

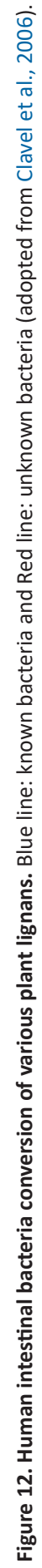
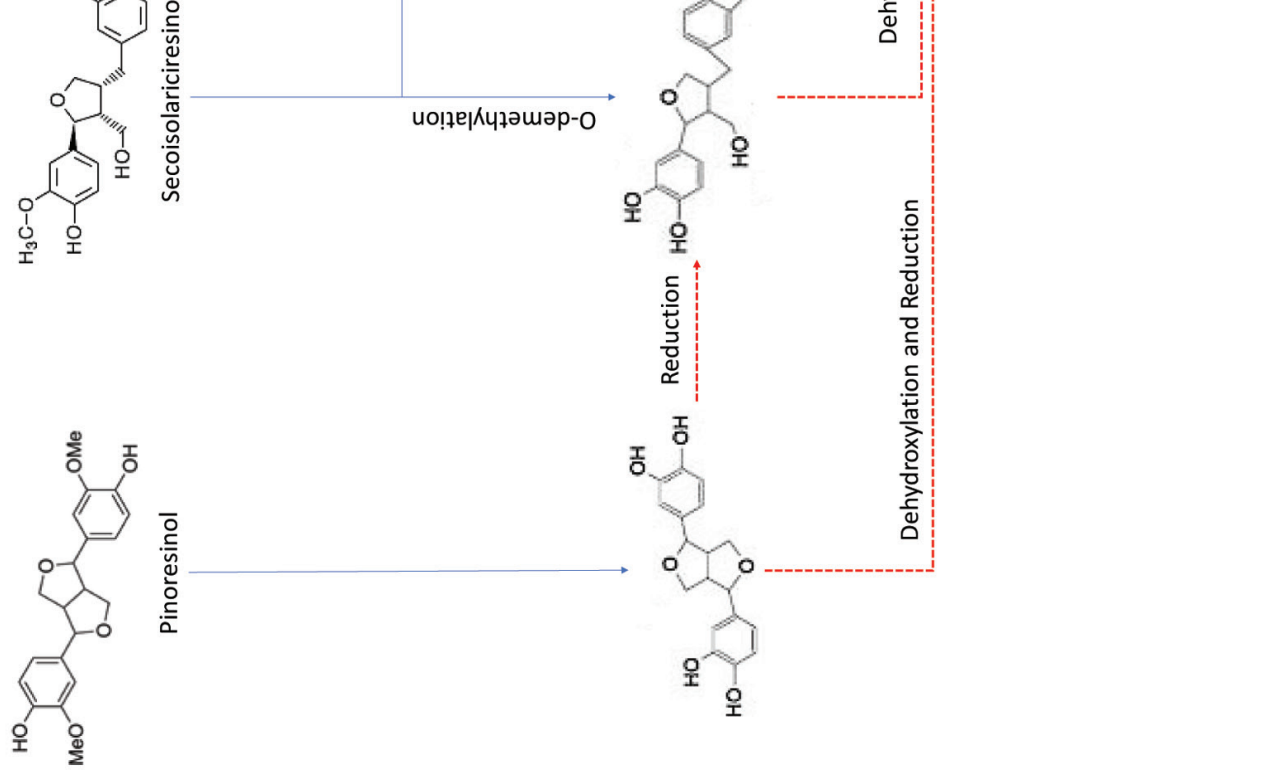
$0.2\left(\times 10^{-6}\right) \mathrm{cm} / \mathrm{s}$, respectively, whereas efflux ratios were $0.8-1.2$, consistent with passive diffusion. This study also found that permeation of secoisolariciresinol diglucoside did not occur as it was not found in the analysis. The conjugation studies also revealed that the extent of conjugation of lignans corresponded to the order of their increasing lipophilicity (secoisolariciresinol diglucoside $<$ secoisolariciresinol $<$ enterodiol $<$ enterolactone). After $48 \mathrm{~h}$, the extent of conjugation for secoisolariciresinol diglucoside, secoisolariciresinol, enterodiol and enterolactone was $<3, \sim 95, \sim 90$ and $>99 \%$, respectively.

\subsection{Health effects of polyphenols}

\subsubsection{Gallic acid}

Gallic acid is known to exhibit antioxidant, anti-inflammatory and cytoprotective effects. Sameermahmood et al. (2010) studied the antiapoptotic effects of gallic acid in RINm5F $\beta$ cells. In this study, the RINm5F $\beta$ cells were exposed to a high concentration of glucose $(25 \mathrm{mM})$ or with palmitate $(500 \mu \mathrm{m})$ or by using a combination of both for $24 \mathrm{~h}$. The experiment was carried out in the presence and absence of gallic acid. The results from DNA damage study using comet assay revealed that cells treated with gallic acid resisted DNA damage and the RINm5F cells under glucolipotoxicity conditions which was decreased considerably by gallic acid. The presence of gallic acid in the system also resulted in increased insulin secretion in RINm5F cells in a dose dependent manner (0.3-10 $\mu \mathrm{M}$ concentration) by counteracting glucolipotoxic effect of lower insulin secretion. In addition, the results from this study indicated that gallic acid prevented apoptosis by upregulating Bcl2 and downregulating caspase-3 signals and decreased NF- $\mathrm{KB}$, caspase and UCP-2 signals.

$\mathrm{Lu}$ et al. (2006b) studied the antioxidant ability and neuroprotective effects of gallic acid derivatives in human SH-SY5Y cells. The free radical scavenging effects of gallic acid in liposome and anti-apoptotic activities in human SH-SY5Y cells induced by 6-hydrodopamine autooxidation were examined in this study. The results so obtained indicated that hydrophobicity of polyphenols play an important role in the antioxidant activity within the cell systems. The neuroprotective effect of a polyphenol compound on a cell against oxidative stress damage depends on two major factors: (a) the capacity to scavenging free radicals and (b) its hydrophobic property that allows it to cross cell membranes to reach its targets. The study after analysis of cell apoptosis, intracellular GSH levels, production of ROS and the influx of $\mathrm{Ca}^{2+}$ concluded that protective effects of gallic acid derivatives in cell systems under oxidative stress depend on both their antioxidant capacities and hydrophobicity. In addition, the neuroprotective effects of gallic acid derivatives seem to depend more on their molecular polarities rather than antioxidant activities in the human SH-SY5Y cell line.

Kaur et al. (2009) studied the anti-proliferative, pro-apoptotic and anti-tumorigenic effects of gallic acid against androgen-independent DU145 and androgen-dependent-22Rv1 human prostate cancer $(\mathrm{PCa})$ cells in nude mice. For this study, athymic male mice (4 weeks old) were used and the mice were subcutaneously injected with $22 \mathrm{Rv} 1$ or DU145 cells into their right flank. The next day the mice were fed with 0.3 and $1 \%(\mathrm{w} / \mathrm{v})$ gallic acid in drinking water. The treatment of DU145 and 22Rv1 cells with gallic acid at the concentrations ranging from 10 to $100 \mu \mathrm{M}$ for $12-48 \mathrm{~h}$ resulted in a concentration- and time-dependent decrease in the viability of cells. The results from MTT assay revealed that gallic acid is selectively toxic to prostate carcinoma cells as compared to non- neoplastic prostate epithelial cells. The study also indicated that treatment of DU145 cell with gallic acid at 50 and $75 \mu \mathrm{M}$ gallic acid resulted in 73 and $84 \%$ apoptotic death and with $22 \mathrm{Rv} 1$ cells with gallic acid at 50 and $75 \mu \mathrm{M}$ resulted in 52 and $73 \%$ apoptotic death after $24 \mathrm{~h}$, respectively. The results from in vivo efficacy of gallic acid in prostate tumor (DU145 and 22Rv1) mouse xenograft model demonstrated that daily oral administration of gallic acid in drinking water at 0.3 and $1 \%(\mathrm{w} / \mathrm{v})$ dose levels for six weeks caused inhibition in the growth of both DU145 and 22Rv1 tumor xenografts. The immunohistochemical analysis from this study revealed significant inhibition of tumor cell proliferation, induction of apoptosis, and reduction of microvessel density in tumor xenografts from gallic acid-fed mice as compared to the control mice.

\subsubsection{Apigenin}

Apigenin is classified as a class II drug of Biopharmaceutical Classification System due to its low solubility and high permeability. Apigenin is poorly soluble in water (solubility $=15.60 \mu \mathrm{g} / \mathrm{ml}$ at ambient temperature) and aqueous buffer (Zhang et al., 2012). Recent oral bioavailability studies in rats were very low at around $7.06 \%$. To improve the solubility and bioavailability of apigenin, various drug delivery techniques such as solid dispersions, microemulsions, liposomes, ethosomes, phytosomes, tranderosomes, nanocrystals, nanocapsule, polymeric micelles, nanoparticles, cyclodextrin complexation, spray dried micropartrticles, dendrimers and self micro emulsifying drug delivery systems have been reported (Alshehri et al., 2019). Apigenin has demonstrated a broad-spectrum of anti-cancer activities including colon-rectal cancer, breast cancer, liver cancer, lung cancer, melanoma, prostate cancer and osteosarcoma. Various strategies are implemented to treat cancer cells by triggering cell apoptosis, inhibit cancer cell proliferation by induction of cell cycle arrest, regulation of cell cycle, inhibition of cell cycle migration and invasion, stimulation of the immune response of patients. Various literature studies have reported apigenin with antitumor activities both in vivo and in vitro (Yan et al., 2017). Apigenin was subjected to treat human lung cancer (H460) cells for a different time to study morphological changes, induction of apoptosis, protein levels associated with apoptosis, dose-dependent apoptosis. The results from the study indicated that apigenin-induced $\mathrm{ROS}$ and $\mathrm{Ca}^{2+}$ production in $\mathrm{H} 460$ cells ( $\mathrm{Lu}$ et al., 2011). In a different study, apigenin inhibited A549 lung cancer cell proliferation and vascular endothelial growth factor (VEGR) transcriptional activation through hypoxiainducible factor 1 (HIF-1) binding site and specifically decreased HIF- $1 \alpha$ in a dose-dependent manner (Liu et al., 2005). Human cancer cells are normally two types: benign and malignant tumors. The benign tumors form in the primary site of a tumor and can be surgically removed. However, the malignant tumors are unstable and can metastasize and invade other tissues. The malignant tumors are very resistant to traditional chemotherapy and radiotherapy. Various studies have indicated that apigenin is also able to work in a combination therapeutic strategy with chemotherapy drugs such as cisplatin and paclitaxel to treat cancer cells (Xu et al., 2011; Yan et al., 2017). Apigenin was able to suppress the proliferation of melanoma cells (A375 and C8161 cell lines) in vitro and to inhibit cell migration and invasion, lengthen the dendrites, and induce G2/M phase arrest and apoptosis (Zhao et al., 2017). Apigenin was used to treat DU145 prostate cancer cells, and the results indicated that apigenin was able to induce cell death and inhibited the migration and invasion of DU145 cancer cells in a dose-dependent manner. Apigenin also led to a reversal of epithelial-mesenchymal transition (EMT) and G2/M phase 
arrest (Zhu et al., 2015). It is reported that apigenin extracted in the form of apigenin 7-O- $\beta$-D-glucopyranoside and apigenin 7-O$\beta$-D-(4'caffeoyl) glucuronide from two medicinal herbs Kummerowia striata and Chrysanthemum morifolium, respectively, have shown anti-HIV activity (Ali et al., 2017; Lee et al., 2003; Tang et al., 1994). Apigenin is also reported to have various other health effects such as antioxidant, anti-inflammatory, blood pressure regulation, antibacterial, and plethora of pharmacological activities against auto-immune disorders, rheumatoid arthritis, multiple sclerosis, Parkinson's disease and Alzheimer's disease (Ali et al., 2017; Yan et al., 2017).

\subsubsection{Acacetin}

Acacetin has been reported in various studies to exhibit anticancer effect by inhibiting cell proliferation and cell cycle progression in human cancer cells, suppressing invasion and migration of cancer cells. The effect of acacetin was studied against two ovarian cancer cells (OVCAR-3 and A2780) and the results indicated that acacetin decreased the steady level of VEGF mRNA and inhibited VEGF transcriptional activation. Acacetin also inhibited HIF- $1 \alpha$ expression and AKT (protein kinase B) activation in a dose dependant manner (Liu et al., 2011). Angiogenesis plays a major role in tumor growth and converts a dormant tumor into a metastatic progression; therefore it is a potential target in cancer prevention and control. Acacetin has angiopreventive efficacy and shown to inhibit the growth of cancer cells, in in vitro, ex vivo and in vivo models. Some of the major attributes of acacetin in preventing angiogenesis includes strongly suppressing human umbilical vein endothelial cell (HUVEC) proliferation and survival and induction of cell death in regular growth conditions and in hypoxia. Acacetin also strongly inhibited vascular endothelial growth factor (VEGF)induced HUVEC proliferation and survival. The results from the study inferred that acacetin has the potential to suppress angiogenesis in response to angiogenic stimuli (Bhat et al., 2013). Hsu et al. (2004) studied the anti-proliferative activity of acacetin and its effect on cell cycle distribution and apoptosis in human lung cancer cell lines (A549). The cell was treated at various doses of $0-20 \mu \mathrm{M}$ for $12-72 \mathrm{~h}$. The normal $\mathrm{p} 53$ gene is reported to play a crucial role in inducing cell apoptosis and cell cycle checkpoints in human and murine cells. The absence of the p53 gene leads to an enhanced risk of carcinogenesis. The A549 cells treated with acacetin resulted in the induction of p53 regulation, cell cycle arrest and triggered apoptosis. FaS/APO-1 protein expressed in the FAS gene is responsible for apoptosis in normal human cells. However, the FaS expression is severely affected in cancerous cells. In this study the acacetin treated A549 cells resulted in increased FaS expression restoring apoptotic sensitivity for natural immune system or chemotherapy. Acacetin efficacy was studied and compared against two other flavonoid compounds, namely linarin and linarin acetate against human prostate cancer cells. The results from that study indicated that acacetin was very efficient and produced excellent growth inhibitory effect through G1 and G2-M cell cycle arrest compared to the other two flavonoids. The efficiency of acacetin decreased when its flavone was modified by disaccharide rhamnose substitution at $\mathrm{C} 7$ for linarin or acetylation of this substituted group for linarin acetate. This study also demonstrated the importance of structural determinants in anticancer efficacy and mechanisms (Singh et al., 2005). It is also reported that acacetin was able to inhibit the invasion and migration of human non-small cell lung cancer A549 cells by suppressing the p38 $\alpha$ MAPK (p37 mitogen-activated protein kinase) signalling pathway (Chien et al., 2011).

\subsubsection{Luteolin and diosmetin}

Among the flavones, luteolin is a potent anti-inflammatory, antioxidant and anti-allergic flavonoid. There are also several reports on its anti-carcinogenic effects and mechanisms. The mechanisms related to anti-inflammatory effects of luteolin includes: (a) inhibition of inducible nitric oxygen synthase (iNOS) expression and NO production, (b) scavenging of ROS, inhibition of ROS production and antioxidant enzymes activation, (c) inhibition of leukotriene production and release, (d) pro-inflammatory cytokine expression suppression, (e) NFkB, Akt, MAPK pathway inhibition, (f) inhibition of adhesion molecule membrane binding, $(\mathrm{g})$ inhibition of hyaluronidase and elastase activity, (h) stabilization of mast cells, and (i) reduction of vascular permeability and modulation of cell membrane fluidity (Seelinger et al., 2008a). Antioxidant efficiency of various phenolics extracted from Olea europaea L. was studied, and luteolin's efficiency was compared against them. The results indicated that luteolin was a potent antioxidant; however, its antioxidant capacity is less than rutin and catechin. The sequence of relative radical scavenging abilities reported in the study is: rutin $>$ catechin luteolin $>\mathrm{OL} \cong$ hydroxytyrosol $>$ diosmetin $>$ caffeic acid $>$ verbascoside $>$ oleuropein $>$ luteolin-7-glucoside $\cong$ vanillic acid $\cong$ diosmetin-7-glucoside $>$ apigenin-7-glucoside $>$ tyrosol $>$ vanillin (Benavente-García et al., 2000). Several anti-carcinogenic activities of luteolin is reported to include: inhibition of proliferating action of various tumor cell lines, induction of cell cycle arrest, inhibition of bFGF and VEGF induced angiogenesis, inhibition of growth tumors, inhibition of $\mathrm{N}$-acetyltransferase in cancer cell line, downregulation of androgen receptor, induction of apoptosisinducing factor and several other activities and mechanism similar to other flavones (Seelinger et al., 2008b).

Pharmacological properties of diosmetin include anti-cancer, anti-microbial, antioxidant, oestrogenic and anti-inflammatory activities. Diosmin is enzymatically hydrolyzed into its aglycone diosmetin and its sugar moiety immediately after oral administration for internal absorption into the body. Anti-cancer activities on carcinoma U251 cell lines, MCF7 breast carcinoma cell lines, T-47D estrogen-receptor-positive breast cancer cell lines, reduction of M-CSF induced proliferation, inhibition of nuclear factor (NF)-kappa-B pathway have been reported (Androutsopoulos et al., 2009; Patel et al., 2013). Diosmin extracted from Rosmarinus officinalis expressed higher antioxidant activities in DPPH assays and produced moderate antibacterial activities against Escherichia coli, Yersinia pestis, Streptococcus pneumoniae, Bacillus anthracis and Bacillus subtilis (Akroum et al., 2017).

\subsubsection{Chrysoeriol}

Like other flavones, chrysoeriol (luteolin 3'-methyl ether) is known to exhibit antioxidant, anti-inflammatory, anti-tumor, antimicrobial, anti-viral activities. Khan and Gilani (2006) studied the effect of chrysoeriol from rooibos tea in in vivo blood pressure experiments on rabbit jejunum, rabbit aorta and guinea-pig trachea. The results indicated that chrysoeriol was able to demonstrate the $\mathrm{K}_{\text {ATP }}$ opening effect in the rabbit jejunum. However, chrysoeriol was 25 times more effective in pig trachea and 40 times more effective in rabbit aorta in a dose-dependent manner. In a different study, chrysoeriol and its glycoside (chrysoeriol-6- $O$-acetyl-4'- $\beta$ D-glucoside) were extracted from Coronopus didymus and its ability to inhibit lipid peroxidation induced by $\gamma$-radiation, Fe (III) and $\mathrm{Fe}$ (II). The result showed a better lipid oxidation protecting effect for chrysoeriol than its glycoside (Mishra et al., 2003). Protective 
effects of chrysoeriol extracted from Eurya cilliata was studied on murine osteoblastic MC3T3-E1 cells. The in vitro studies indicated that chrysoeriol increased osteoblastic differentiation and protected against $\mathrm{H}_{2} \mathrm{O}_{2}$ induced oxidative stress and induced toxicity. The results also indicated that chyroesiol may help promote bone recovery from inflammatory bone diseases (Kim et al., 2010). Chyrsoeriol extracted from the leaves of Digitalis purpurea was studied to know its ability to inhibit nitric oxide synthase in Raw 264.7 macrophage cell line. The results indicated that chrysoeriol pre-treatment of the cells inhibited the release of nitric oxide (NO) in the cells treated with lipopolysaccharide (LPS) and it also inhibited LPS-induced inductions of the iNOS gene. The mechanism behind these induction capabilities of chrysoeriol is that it selectively suppressed AP-1 activation (Choi et al., 2005).

\subsubsection{Quercetin and kaempferol}

Quercetin contains various physiological properties including anti-inflammatory, antioxidant, psychostimulant, cardioprotectant, ant-cancer, anti-viral and neuroprotective effects. Oh et al. (2019) reported that quercetin and its ester showed antioxidant acitivy in $\mathrm{DPPH}$ and ABTS radical scavenging assays. Anti-hypertensive effects of quercetin with an oral daily dose of $10 \mathrm{mg} / \mathrm{kg}$ in spontaneous hypertensive (SHR) rats and normotensive Wistar Kyoto rats (WKY) were analyzed. The results indicated that quercetin reduced the elevated blood pressure, cardiac and renal hypertrophy and functional vascular changes in SHR rats without any change in WKY rats. These effects validate the antioxidant properties of quercetin (Duarte et al., 2001). The anti-inflammatory, effects of quercetin on cardiovascular risk markers including human C-reactive protein (CRP) and anti-atherosclerosis effect using transgenic humanized models of cardiovascular disease was also studied. The results from this study indicated that quercetin reduces the expression of human CRP and cardiovascular risk factors (SAA, fibrinogen) in mice in vivo. It was also suggested that local anti-proliferative and anti-inflammatory effects in the aorta may contribute to the attenuation of atherosclerosis (Kleemann et al., 2011). Quercetin's role in protecting H9c2 cells against oxidative stress was studied and it was reported that quercetin was able to reduce intercellular ROS production and $\mathrm{H}_{2} \mathrm{O}_{2}$ cell damage. In addition, quercetin was able to modulate pro-survival signalling through ERK1/2 and PI3K/Akt pathways (Angeloni et al., 2007).

Kaempferol is a flavonol, which exhibits anti-inflammatory, anti-cancer, anti-allergic, anti-fungal and spasmolytic properties. In addition, it stabilizes the structure of connective tissue and strengthen the walls of blood vessels. It is reported to reduce the level of glucose in blood and inhibit the activity of aldose reductase by preventing neuropathy and retinopathy (Palacz-Wróbel, 2018). Human gut (HuTu-80 and Caco-2) and breast cancer cells (PMC42) were used to show the synergistic effect of quercetin and kaempferol in reducing cell proliferation. The results indicated that a single dose of combined quercetin and kaempferol at $10 \mu \mathrm{M}$ showed greatest reduction in cell proliferation in Caco-3 cells. Furthermore, 4-days and 14-days exposure to quercetin and kaempferol was also effective in reducing proliferation of Caco-2, HuTu- 80 and PMC42 cells. The combination effect of quercetin/kaempferol was better than individual action of cancer cells (Ackland et al., 2005). Kaempferol modulated several key factors in signal transduction pathways linked to apoptosis, angiogenesis, inflammation and metastasis. Various studies have revealed that there is an inverse relationship between kaempferol intake and cancer cells. Furthermore, kaempferol not only induces cancer cell apoptosis but also preserves cell viability by exhibiting cell protective effects
(Chen and Chen, 2013). Like other flavonols, myricetin, isorhamnetin, tamarixetin, morin and fisetin have been reported to exhibit distinctive antioxidant and anti-inflammatory effects, as well as cytotoxic effects on various human cancer cells, anti-inflammatory and cardioprotective effects (Erlejman et al., 2004; Jaramillo et al., 2010; Morales and Haza, 2012; Subash and Subramanian, 2009; Wang et al., 2006; Zieliñska et al., 2001).

\subsubsection{Diadzein and genistein}

Diadzein as an isoflavone phytoestrogen is metabolised to equol and $O$-desmethylangolensin. Genistein is metabolised to (-)-5-hydroxyequol and 5-hydroxydehydroequol. Several studies have suggested that both equol and $O$-desmethylangolensin have more extensive biological properties than the parent compound itself. Mathey et al. (2007) studied the bioavailability of genistein, diadzein and equol in rats which were ovariectomised and the effect of bone health was studied based on feeding studies. In this study, the rats were fed with a control diet that was supplemented with genistein, diadzein or equol at $10 \mu \mathrm{g} / \mathrm{g}$ body weight/day for 3 months. In addition, some rats were also fed with short chain fructooligosaccharides or with live microbial Lactobacillus casei together with diadzein as precursor. In this study, genistein, daidzein or equol exhibited a bone sparing effect and the total femoral bone mineral density (BMD) was significantly enhanced when compared to that of rats fed with control diet, as was the metaphyseal compartment. The results suggested that bone strength was improved by consuming equol alone in the diet, but it was not the case when consuming genistein or diadzein alone. The effect of addition of short chain fructooligosaccharides to the diet significantly enhanced the protective efficiency of diadzein on femoral bone mineral density and mechanical properties. Therefore, long term equol consumption like genistein and daidzein, in the ovariectomized rat, provides bone sparing effects and addition of short chain fructooligosaccharides or live microbial Lactobacillus casei together with diadzein improved the protective effects of daidzein on the skeleton.

It is reported that equol is more bioavailable than diadzein or genistein. Setchell et al. (2009) studied the pharmacokinetics of natural S-(-) equol after administration single-bolus oral doses of 10 and $30 \mathrm{mg}$ in the form of SE5-OH tablet to healthy postmenopausal women. S-(-) equol concentrations was measured in plasma and urine collected at timed intervals over a 48 -h period post-dosing using tandem MS. The results indicated that equol was rapidly absorbed and attained high plasma concentrations and showed a linear dose response in its pharmacokinetics with a plasma elimination half-life of $8 \mathrm{~h}$. The systemic bioavailability of equol was very high and the fraction of dose excreted in urine $\left(\% \mathrm{f}_{\mathrm{e}, \mathrm{u}}\right)$ was $82 \%$, which is greater than published data for daidzein and genistein. Lund et al. (2011) studied the effect of equol for improved prostate health via in vitro and in vivo studies. The study evaluated the effects of $5 \alpha$-DHT or equol alone and in combination on prostate specific antigen (PSA) by LNCap human prostate cancer cells. The result from the in vitro study indicated that equol binds specifically $5 \alpha$-DHT and prevents increases in PSA from LNCap cells. The in vivo studies indicated that equol decreases rat prostate size, decreases serum $5 \alpha$-DHT levels and androgen hormone action. The study concluded that equol can play an important role in maintaining prostate health in men without altering other circulating sex steroids or LH levels. In addition, it is also reported that equol has antioxidant, anti-proliferative, vasorelaxant and antiinflammatory properties (Blay et al., 2010; Hedlund et al., 2003; Jackman et al., 2007; Rimbach et al., 2003; Shor et al., 2012). 


\subsubsection{Hesperetin}

Hesperetin, one of the most abundant flavonoids found in citrus fruits is reported to demonstrate a wide spectrum of pharmacological effects such as anti-inflammatory, anti-carcinogenic, anti-hypertensive, anti-atherogenic and antioxidant properties (Banjerdpongchai et al., 2016; Choi, 2008; Haidari et al., 2009; Hajialyani et al., 2019; Ikemura et al., 2012; Lee et al., 2012; Milenkovic et al., 2011; Parhiz et al., 2015; Pari and Shagirtha, 2012; Wilmsen et al., 2005; Yamamoto et al., 2013). Ikemura et al. (2012) studied the effect of hesperidin, glucosyl hesperidin and naringin on hypertension and cerebral thrombosis in stroke prone hypertensive rats. Hesperidin, glucosyl hesperidin and naringin was mixed with the powder diet at various concentrations and the control diet was unmodified and the rats were fed for 4 weeks. The results indicated that there was no effect on the body weight, however, the supplemented diet significantly suppressed the age related increase in blood pressure. The thrombotic tendency was determined by He$\mathrm{Ne}$ laser technique in the cerebral blood vessels, in which the number of laser pulses required to generate an occlusive thrombus was used as an index. The results indicated that thrombotic tendency of the hypertensive rats was decreased signifcantly comparted to the control diet fed rats. The effect of hesperidin, glucosyl hesperidin and naringin on oxidative stress was determined by the concentration of 8- hydroxy-2'-deoxyguanosine (8-OHdG) that demonstrated the supplements had strong antioxidant activity. Furthermore, these supplements significantly increased the production of nitric oxide (NO) metabolites in urine measured with Griess reagent. Yamamoto et al. (2013) studied the effects of continuous ingestion of hesperidin and glucosyl hesperidin on vascular gene expression in spontaneously hypertensive rats. The results from this study indicated that continous ingestion of hesperidin and glucosyl hesperidin for 8 weeks significantly decreased the hypertension and suppressed the mRNA expression of NADPH oxidase subunits and thromboxane. The continous ingestion alters the gene expression of vascular regulatory molecules in the aorta and support the notion that fruit flavonoids have beneficial effects on vasculature.

Banjerdpongchai et al. (2016) studied various flavanones in citrus seed extracts for their cytotoxic effect, mode of cell death, and signaling pathway in human hepatocellular cancer HepG2 cells. Neohesperidin, hesperidin, and naringin, active flavanone glycosides were identified in citrus seed extract. The cytotoxic effect of all three compounds were studied in a dose depedent manner at $\mathrm{IC}_{0}, \mathrm{IC}_{10}, \mathrm{IC}_{20}$, and $\mathrm{IC}_{50}$ for $24 \mathrm{~h}$ and the cells were visualized after annexin V-fluorescein isothiocyanate and propidium iodide staining using flow cytometry. The results indicated that hesperidin did not induce reactive oxygen species and caspase- $9,-8$ and -3 activities were activated and increased in the hesperidin treated HepG2 cells. The results also indicated that expression of proapoptotic Bax, Bak, and $\mathrm{tBid}$ proteins was higher whereas the $\mathrm{Bcl}-\mathrm{xL}$ and proform of Bid protein levels were downregulated. The study concluded that hesperidin induced human HepG2 cell apoptosis via mitochondrial pathway and death receptor pathway. Lee et al. (2012) studied the effect of hesperidin to induce apoptosis in human malignant pleural mesothelioma (MSTO-211H) cells. In this study, the MSTO- $211 \mathrm{H}$ cells were treated with hesperidin at various concentrations $(40,80$, and $160 \mu \mathrm{M})$ and control samples were untreated for 24 and $48 \mathrm{~h}$. The results indicated that the $\mathrm{IC}_{50}$ value of hesperidin was $152.3 \mu \mathrm{M}$ in MSTO-211H cells for $48 \mathrm{~h}$ and hesperidin decreased cell viability, and induced apoptotic cell death. The study also demonstrated that hesperidin significantly suppressed the mRNA/protein level of specificity protein $1(\mathrm{Sp} 1)$ and modulated the expression level of the $\mathrm{Sp} 1$ regulatory protein such as p27, p21, cyclin D1, Mcl-1, and survivin in mesothelioma cells.

\subsubsection{Naringenin}

Naringin and its aglycone naringenin are reported to exhibit various bioactivities such as anti-inflammatory, neuroprotection, antifibrogenic, antioxidant and antioxidant activities. Heo et al. (2004) studied the effect of antioxidant naringenin from Citrus junos on the neuroprotection. It is believed that generation of ROS and oxidative damage are involved in the pathogenesis of neurogenerative disorders. Furthermore, amyloid $\beta$ protein $(\mathrm{A} \beta)$ induced free radical-mediated neurotoxicity is one of the leading causes of Alzheimer's disease. In this study, the protective effect of naringenin from Citrus junos against $\mathrm{A} \beta$-induced neurotoxicity was studied using pheochromocytoma cells (PC12 cells). The cells pretreated with naringenin $(25-100 \mu \mathrm{M})$ and vitamin $\mathrm{C}(100 \mu \mathrm{M})$ resulted in the prevention of generation of $\mathrm{A} \beta$-induced reactive oxygen species. In addition, naringenin treatment led to a decrease in $\mathrm{A} \beta$ toxicity in a dose-dependent manner which was assessed by the 3-(4,5-dimethylthiazol-2-yl)-2,5-diphenyltetrazolium bromide assay. To test the effect of naringenin, scopolamine-induced in vivo method with a passive avoidance test was carried out using ICR mice, administered with $4.5 \mathrm{mg} / \mathrm{kg}$ body weight of naringenin and scopolamine at $1 \mathrm{mg} / \mathrm{kg}$ body weight was used. The results from this test showed significant anti-amnesic effects in mice, thereby demonstrating neuronal cell protection by naringenin.

Naringenin has beneficial effects against liver diseases via several molecular mechanisms. The main protective of naringenin in liver diseases include (a) inhibition of oxidative stress, (b) transforming growth factor (TGF- $\beta$ ) pathway, (c) inhibition of vascular endothelial growth factor (VEGF), (d) the prevention of the trans differentiation of hepatic stellate cells (HSC), leading to decreased collagen synthesis, (e) inhibition of the mitogen activated protein kinase (MAPK), toll-like receptor (TLR) and TGF- $\beta$ non-canonical pathways, and (f) regulation of lipid metabolism by modulating the synthesis and oxidation of lipids and cholesterol (HernándezAquino and Muriel, 2018).

La et al. (2009) studied the effect of naringenin on human osteoclastogenesis osteoclastic bone resorption. The study was carried out using primary osteoclast precursor cells activated by receptor activator of nuclear factor-kB ligand (RANKL) and macrophage colony-stimulating factor (M-CSF) for 6 days. The cells were treated with 10,25 or $50 \mu \mathrm{g} / \mathrm{mL}$ of naringenin and the control samples were run without naringenin. The osteoclast formation was determined by the number of tartrate-resistant acid phosphatase (TRAP)-stained multinuclear cells. The cytokine secretion from osteoclast precursor cells incubated with RANKL and M-CSF in the absence or presence of naringenin $(10 \mu \mathrm{g} / \mathrm{mL})$ for 6 days to determine the concentrations of interleukin (IL)-1 $\alpha$, IL-1 $\beta$, IL-6, IL-8, IL-17, IL-23, monocyte chemoattractant protein-1 (MCP-1), osteoprotegerin (OPG) and TNF- $\alpha$, using sandwich enzyme-linked immunosorbent assays. The results from this study indicated that $50 \mu \mathrm{g} / \mathrm{ml}$ of naringenin inhibited osteoclastogenesis by $96 \%$. In addition, naringenin also significantly inhibited the secretion of interleukin (IL)- $1 \alpha$ (by $59 \%$ ), IL-23 (by $87 \%$ ) and monocyte chemoattractant protein-1 (by $58 \%$ ). This results from this study demonstrated that naringenin can inhibit human osteoclastogenesis and osteoclastic bone resorption and has the potential to be used as a therapeutic or preventive agent for bone-related diseases such as periodontitis.

Wang et al. (2012) investigated the role of naringenin against lead-induced oxidative stress in liver and kidney of rats. For this study, the researchers orally administered lead acetate $(500 \mathrm{mg}$ 
$\mathrm{Pb} / \mathrm{L}$ ) to adult male Sprague-Dawley rats for 8 weeks to induce hepatoxicity and nephrotoxicity. During this study, the results indicated increases in the levels of alanine aminotransferase, aspartate aminotransferase, urea, uric acid, and creatinine after lead administration in rats. In addition, there were significant decreases in various antioxidant enzymes such as GSH glutathione, SOD superoxide dismutase, CAT catalase, GPx glutathione peroxidases in the lead treated rats. These enzymes are important in defense against superoxide radicals and $\mathrm{H}_{2} \mathrm{O}_{2}$. The rats which are affected by lead and treated by naringenin exhibited increased activities of $\mathrm{OD}, \mathrm{CAT}$, and GPx in the liver and kidney due to the ability of naringenin to reduce the accumulation of free radicals generated during lead-induced lipid peroxidation.

\subsubsection{Catechins}

Catechins are major flavan-3-ol found in various cocoa extracts and tea extracts, with demonstrated bioactivities for human such as cardiovascular health benefits, antioxidant activity, anti-inflammatory effect, improved skeletal muscle structure and endothelial function (Zhong et al., 2012; Chiu et al., 2018; Perera et al., 2018; Li et al., 2019; Ambigaipalan et al., 2020). Spadafranca et al. (2010) studied the effect of dark chocolate on plasma epicatechin levels, DNA resistance to oxidative stress and total antioxidant activity in healthy subjects. The dietary intervention study was conducted with 20 healthy individuals (ten men and ten women) for 4 weeks. Of the twenty volunteers, five men and five women volunteered to consume dark chocolate and the remainder chose to eat white chocolate. Volunteers were advised to consume chocolate as a snack with $40 \mathrm{~g}$ white bread. The blood samples were collected at the beginning of the experiment at various time intervals during the $24 \mathrm{~h}$ period. The results from this study indicated that dark chocolate as a snack along with balance diet can improve DNA resistance to oxidative stress in healthy subjects. However, there was no beneficial effect found in consuming dark chocolate regularly compared to a single occasional intake. These results suggest that DNA protection offered by consuming dark chocolate against chronic degenerative disease is still with an unknown mechanism. Gutierrez-Salmean et al. (2014) studied the effects of epicatechin on molecular modulators of skeletal muscle growth and differentiation. In this study, the effects of aging on protein levels of recognized modulators of skeletal muscle (SkM) growth (myostatin, follistatin), senescence-associated $\beta$-galactosidase (SA- $\beta$-Gal) and myogenic differentiation (myogenin, MyoD, MEF2A, Myf5) in young and old mice were investigated. During the study, mice were fed with $1 \mathrm{mg} / \mathrm{kg}$ BID. The human subjects in this work were treated for 7 days with $25 \mathrm{mg}$ pure epicatechin in the capsule form at $1 \mathrm{mg} / \mathrm{kg} /$ day concentration. The muscle strength was assessed by handgrip dynamometry. The blood samples were collected before and after treatment. The results from this mice study indicated that the markers important for ageing such as myostatin and senescence-associated $\beta$-galactosidase levels which were downregulated, while the markers associated with the muscle strength such as follistatin and Myf5 were increased after consumption of epicatechin. In human study, treatment with epicatechin increased the handgrip strength and the ratio of plasma follistatin/myostatin.

Engler et al. (2004) studied the effect of dark chocolate intake on endothelial function. The study was randomized, double-blind, placebo-controlled design conducted over a 2-week period in 21 healthy adult subjects. The subjects were randomly assigned to daily intake of high-flavonoid (213 mg procyanidins, $46 \mathrm{mg}$ epicatechin) or low-flavonoid dark chocolate bars (46 g, $1.6 \mathrm{oz})$. The flow-mediated dilation of the brachial artery was compared be- tween the low flavonoid chocolate and high-flavonoid chocolate group. The dilation of branchial artery is an endothelial dependent function associated with the release of nitric oxide and endothelium-derived prostanoids. The results indicated elevated plasma epicatechin concentrations in the high-flavonoid group increased endothelium-derived vasodilators and improved endothelial function. The results also suggested that there was no change in oxidative stress measures, lipid profiles, blood pressure, body weight or BMI between two groups. Schroeter et al. (2006) indicated that ingestion of flavanol rich cocoa resulted in a significant elevation of circulating NO species, enhanced flow-mediated dilation response in conduit arteries and augmented microcirculation. In addition, oral administration of pure epicatechin to humans closely emulated acute vascular effects of flavanol-rich cocoa. The data from this study indicated that epicatechin is linked to the reported vascular effects observed after the consumption of flavanol-rich cocoa.

Cremonini et al. (2016) evaluated the effects of a high fat diet (HFD) and epicatechin (EC) consumption on the activation of the insulin cascade and its negative modulators were evaluated. The results revealed that consumption of a high fat diet for 15 weeks caused obesity and insulin resistance in C57BL/6J mice as demonstrated by high fasted and fed plasma glucose and insulin levels, and impaired insulin tolerance tests (ITT) and glucose tolerance tests (GTT). This mechanism was attributed to the alterations in the activation of components of the insulin-triggered signaling cascade (insulin receptor, IRS1, ERK1/2, Akt) in adipose and liver tissues. These high fat fed mice were treated with an epicatechin rich diet and the results indicated that epicatechin supplementation showed downregulation of c-Jun N-terminal kinase (JNK), IкB kinase (IKK), protein kinase $\delta(\mathrm{PKC} \delta)$ and protein tyrosine phosphatase 1B (PTP1B), leading to improved insulin sensitivity. Therefore, it was concluded that consumption of EC-rich foods could constitute a dietary strategy to mitigate obesity-associated insulin resistance.

Shin et al. (2009) studied the effect of major green tea polyphenols on the adipocyte differentiation in human bone marrow mesenchymal stem cells (hBM-MSCs) and compared it to the effect of representative antidiabetic drugs. In this study, the human bone marrow mesenchymal stem cells grown in Dulbecco Modified Eagle's Medium (DMEM) with low glucose $(1 \mathrm{~g} / \mathrm{L})$ containing $10 \%$ fetal bovine serum (FBS) and supplemented with antibiotics and glutamax. In order to induce adipose differentiation, the concentration of glucose was increased to $4.5 \mathrm{~g} / \mathrm{L}$ and supplemented with $10 \%$ fetal bovine serum (FBS), $10 \mu \mathrm{g} / \mathrm{mL}$ insulin, $1 \mathrm{mM}$ dexamethasone, $0.5 \mathrm{mM}$ 3-isobutyl-1-methylxanthine (IBMX). The samples were treated with nine different polyphenols such as (-)-catechin, (+)-catechin, (-)-epigallocatechin-3-gallate (EGCG), (-)-epicatechin (EC), (-)-epigallocatechin (EGC); (-)-epicatechin gallate (ECG), (-)-gallocatechin-3-gallate (GCG), (-)-catechin3 -gallate $(\mathrm{CG})$, and (-)-gallocatechin (GC) separately every 2 days, for 6 or 12 days and the results were compared against cells treated with troglitazone, or glibenclamide. The results from the study indicated that (-)-catechin was the most potent of the eight green tea polyphenols evaluated in promoting adipocyte differentiation in human bone marrow mesenchymal stem cells in a dose dependant manner. The cells treated with (-)-catechin had increased the mRNA levels of adipogenic markers, such as adiponectin, peroxisome proliferator-activated receptor gamma (PPAR $\gamma$ ), FABP4, and LPL. In addition, (-)-catechin upregulated the secretion of adiponectin in hBM-MSCs culture. In conclusion, the study suggested that (-)-catechin promoted adipocyte differentiation and increased sensitivity to insulin in part by direct activation of PPAR $\gamma$, which demonstrated the pharmacological benefits of green tea intake in reducing the risk of type 2 diabetes. 
Nagao et al. (2005) investigated the effects of catechins on the blood variables and oxidized-LDL and the association between body fat variables and oxidized-LDL. The body mass index (BMI) correlates with the amount of malondialdehyde and thiobarbituric acid-reactive substances in the blood. In this study, 12-week double-blind study was performed in which the 17 subjects drank 1 bottle oolong tea/day containing $690 \mathrm{mg}$ catechins and a control group containing 18 subjects drank 1 bottle oolong tea/day containing $22 \mathrm{mg}$ catechins. Both the beverages used in this study contained several isomers of this compound catechin, catechin gallate (CG), gallocatechin (GC), gallocatechin gallate (GCG), epicatechin, epicatechin gallate (ECG), epigallocatechin, and epigallocatechin gallate (EGCG) in various concentrations. The results indicated that body weight, BMI, waist circumference, body fat mass, and subcutaneous fat area were significantly lower in the green tea extract group than in the control group. The study concluded that long-term consumption of beverages containing catechins inhibits the formation of oxidized lipids such as malondialdehyde-modified LDL thereby reducing the risk for developing arteriosclerosis. The results from this study also suggest that catechins contribute to the prevention of and improvement in various lifestyle-related diseases, particularly obesity.

$\mathrm{Apc}^{\mathrm{min} /+}$ mice are recognized as a genetically relevant animal model mimicking human intestinal carcinogenesis and it is used in a study by Ju et al. (2005) to investigate the inhibition of intestinal tumorigenesis by ECG present in green tea. The mice used in this study carry a dominant heterozygous nonsense mutation at codon 850 of the mouse homologue of the human adenomatous polyposis coli (APC) gene. The APC gene is a tumor suppressor gene and its mutation is implicated in both sporadic and inherited human colorectal carcinogenesis. During this study, the mice treated with 0.08 and $0.16 \%$ ( 0.16 or 0.08 g ECG dissolved in $100 \mathrm{~mL}$ distilled water containing $0.5 \mathrm{~g}$ citric acid) significantly decreased the small intestinal tumor formation by 37 and $47 \%$, respectively. In the same study, ECG was administered in a dose dependent manner at $0,0.02,0.04,0.08,0.16$, or $0.32 \%$ concentration in drinking fluid for 6 weeks until the study was terminated at 11 weeks of age. This study resulted in the increased levels of E-cadherin and decreased levels of nuclear B-catenin, c-Myc, phospho-Akt, and phospho-extracellular signal-regulated kinase $1 / 2(E R K 1 / 2)$ in small intestinal tumors. Based on these results, ECG was used at 12.5 or $20 \mu \mathrm{mol} / \mathrm{L}$ concentration to treat HT29 human colon cancer cells which resulted in increased E-cadherin protein levels from 27 to $58 \%$, induced the translocation of B-catenin from nucleus to cytoplasm and plasma membrane, and decreased c-Myc and cyclin D1 (Ju et al., 2005).

Lu et al. (2006a) investigated the inhibitory effect of polyphenon E (a standardized green tea polyphenol preparation containing $65 \%$ EGCG and caffeine on 4-(methylnitrosamino)-1-(3-pyridyl)1-butanone (NNK)-induced lung tumor progression from adenoma to adenocarcinoma. For this study, the $\mathrm{A} / \mathrm{J}$ mice were treated with a single dose of NNK at a concentration of $103 \mathrm{mg} / \mathrm{kg}$ bodyweight and kept for 20 weeks for the progression of lung adenomas. Then after 20 weeks, the mice were treated with $0.5 \%$ Polyphenon E or $0.044 \%$ caffeine as the sole source of drinking fluid until $52^{\text {nd }}$ week. Histopathological analysis of the mice indicated that Polyphenon E administration significantly reduced the incidence by $52 \%$ and multiplicity by $63 \%$ of lung adenocarcinoma. The treatment with caffeine also reduced the incidence by $48 \%$ and multiplicity by $49 \%$. The results also indicated that Polyphenon E and caffeine administration inhibited cell proliferation by 57 and $50 \%$, respectively in adenocarcinomas. In addition, the Polyphenon E and caffeine administration enhanced apoptosis by 2.6 - and 4-fold in adenocarcinomas, respectively and adenomas (both by 2.5 - fold), and lowered levels of c-Jun and extracellular signal-regulated kinase (Erk) 1/2 phosphorylation. The study concluded that epigallocatechin-3-gallate was able to suppress the progression of NNK-induced lung adenoma to adenocarcinoma supported with decreased cell proliferation, enhanced apoptosis, and lowered levels of c-Jun and Erk1/2 phosphorylation.

Gupta et al. (2001) studied the inhibition of prostate carcinogenesis using autochthonous transgenic adenocarcinoma of the mouse prostate (TRAMP) model, which spontaneously develops metastatic $\mathrm{CaP}$. This model was chosen by the researchers as it mimics progressive forms of human disease. The polyphenolic dose was isolated from green tea at a human achievable dose equivalent to 6 cups of green tea per day. In the control samples, the mice were fed only with water and after 32 weeks all the 20 mice had palpable tumors and they had various stages of metastases to lymph nodes, lungs, liver and bone. In the test subjects, the mice were treated with $0.1 \%$ polyphenols $(\mathrm{w} / \mathrm{v})$ from 8 to 32 weeks. The results indicated that the polyphenol treated samples had significant delay in primary tumor incidence and tumor burden as assessed sequentially by MRI. In addition, there was significant decrease in prostate $(64 \%)$ and genitourinary (GU) $(72 \%)$ weight. The was significant inhibition in serum insulin-like growth factor-I and restoration of insulin-like growth factor binding protein-3 levels and marked reduction in the protein expression of proliferating cell nuclear antigen (PCNA) in the prostate compared with water-fed TRAMP mice. The study concluded that green tea polyphenol consumption caused significant inhibition of prostate cancer development, progression, and metastasis of $\mathrm{CaP}$ to distant organ sites.

\subsubsection{Proanthocyanidins}

Proanthocyanidins also known as condensed tannins are a group of monomers and polymers of flavanols with no sugar groups attached to them (Chandrasekara and Shahidi, 2010). They occur mainly as dimers and polymers that are bound together by links between $\mathrm{C} 4$ and $\mathrm{C} 8$ or $\mathrm{C} 4$ and $\mathrm{C} 6$ positions. Proanthocyanidins upon depolymerization yield epicatechin under acidic conditions with mean degree of polymerization between 4 and 11. Proanthocyanidins have reported to various health effects greater than flavanols particularly in treating colon cancer (Rossi et al., 2010). Gossé et al. (2005) studied the anti-proliferative mechanisms on human metastatic colon carcinoma (SW620 cells) of apple polyphenol fractions and evaluated the anti-carcinogenic properties in vivo. The study was carried out with two types of polyphenol classes extracted from apples, (a) the non-proanthocyanidin fraction containing $73 \%$ phenolic monomers and (b) the procyanidin fraction containing $78 \%$ procyanidins. The results from the study indicated that only the procyanidin fraction was able to inhibit the SW260 cell growth with an $\mathrm{IC}_{50}$ value of $45 \mu \mathrm{g} / \mathrm{mL}$. Also, after 24 $\mathrm{h}$ exposure to procyanidin fraction, protein kinase $\mathrm{C}$ activity was inhibited by $70 \%$ and a significant increase in extracellular signalregulated kinases 1 and 2 and c-jun $\mathrm{N}$-terminal kinases expression was observed together with the downregulation of polyamine biosynthesis and the activation of caspase- 3 . The in vivo studies were carried using male Wistar rats and the carcinogenesis was induced by intraperitoneal injections of azoxymethane, once a week for 2 weeks. The rats were treated with $0.01 \%$ procyanidin fraction dissolved in drinking water after 7 days and the treatment was continued for 6 weeks. The results after 6 -week treatment indicated that colon of rats showed significant reduction of the number of preneoplastic lesions when compared with controls receiving water. The study concluded that apple procyanidins alter intracellular 
signaling pathways, polyamine biosynthesis and trigger apoptosis in tumor cells.

Prasad et al. (2012) studied the effect of bioactive proanthocyanidins extracted from grapeseed using in vitro and in vivo models. In this study, the in vitro analysis was carried out on human pancreatic cancer cells (Miapaca-2, PANC-1 and AsPC-1). The cells viability and cell death assays were carried out by treating the cancer cells with $50 \mu \mathrm{L}$ of proanthocyanidins $(5 \mathrm{mg} / \mathrm{mL})$ for 24 and $48 \mathrm{~h}$. Miapaca-2 and PANC-1 cells were treated with different concentration of grapeseed proanthocyanidins (GSPs) $(0$, 20,40 and $60 \mathrm{mg} / \mathrm{mL}$ ) in complete medium for $48 \mathrm{~h}$. The result indicated that GSPs reduced cell viability increased G2/M phase arrest of the cell cycle leading to induction of apoptosis in a doseand time-dependent manner. In addition, the results indicated that GSPs induced apoptosis of pancreatic cancer cells were associated with a decrease in the levels of Bcl-2 and Bcl-xl and an increase in the levels of Bax and activated caspase-3. Furthermore, the treatment of Miapaca-2 and PANC-1 cells with GSPs also decreased the levels of phosphatidylinositol-3-kinase (PI3K) and phosphorylation of Akt at ser473. The in vivo study was carried out by subcutaneously injecting Miapaca- 2 cells ( 5 x $10^{6}$ in $100 \mu$ L PBS) in the right flank of each mouse. The mice were administered with $0.5 \%$ GSPs-supplemented AIN76A control diet in pellet form throughout the experiment period after one day of injection. The results after GSP treatment demonstrated inhibition of cell proliferation, induction of apoptosis of tumor cells, increased expression of Bax, reduced expression of anti-apoptotic proteins and activation of caspase-3-positive cells and decreased expression of PI3K and $\mathrm{p}$-Akt in tumor xenograft tissues. Overall, the study concluded that GSPs could potentially be used as chemotherapeutic effect on pancreatic cancer cell growth.

Akhtar et al. (2009) studied the effect of GSPs on human nonsmall cell lung cancer (NSCLC) cells in vitro and in vivo using a tumor xenograft model. During the study, the NSCLC cells were treated with various concentrations $0,20,40,60$, and $80 \mu \mathrm{g} / \mathrm{mL}$ of GSPs for $24 \mathrm{~h}$. The study indicated inhibition of proliferation of cells $(5-17 \%)$; however, significant greater inhibitory effect was observed at $48 \mathrm{~h}$ after GSPs treatment (10-68\%). The study also indicated that GSPs did not inhibit cell proliferation of normal human bronchial epithelial cells. The in vivo treatment with GSPs against human NSCLC tumor xenograft growth after subcutaneous injection in mice was studied at different concentrations $(0.1,0.2$ and $0.5 \% \mathrm{w} / \mathrm{w}$ ). The results demonstrated inhibition of the growth of lung tumor xenografts in nude mice by dietary GSPs is associated with the inhibition of tumor cell proliferation, angiogenesis, and up-regulation of IGFBP-3.

\subsubsection{Resveratrol}

Resveratrol (trans-3,5,4'-trihydroxystilbene) is a naturally occurring polyphenol with various health effects. Oh and Shahidi (2018) reported that resveratrol and its esters inhibited oxidation of DNA and LDL cholesterol effectively. Oh et al. (2019) reported that resveratrol and its derivatives reduced reactive nitrogen species generation in murine microphages and decreased cell viability of liver cancer (HepG2), colon cancer (HT-29, A431), breast cancer (MCF7), and gastric cancer (AGS) cell lines. Asensi et al. (2002) studied the inhibition activity of resveratrol against B16 melanoma (B16M) cells in mice, rats and rabbits. As a part of this study, the researchers carried out pharmacokinetic investigations in which 20 mg of trans-resveratrol (t-RES) were administered orally (via an intragastric tube) to rabbits, rats, and mice or intravenously to rabbits (via the ear vein). The results from this pharmacokinetic study revealed that highest concentration of resveratrol in plasma, either after i.v. or oral administration was reached within the first 5 min in all animals. The amount of resveratrol in extravascular levels (brain, lung, liver, and kidney) were always $<1 \mathrm{nmol} / \mathrm{g}$ fresh tissue. The resveratrol measured in plasma or tissues was in the trans form. In addition, the results indicated that hepatocytes metabolized trans-resveratrol in a dose-dependent fashion and resveratrol was removed from circulation by liver very rapidly. The in vitro testing of resveratrol against B16 melanoma (B16M) cells resulted in inhibition of cell proliferation and reactive oxygen species in a concentration-dependent fashion. The results from in vivo studies on all three animals injected with B16 melanoma (B16M) cells in the footpad revealed that oral administration of resveratrol did not inhibit growth of B16M inoculated into the footpad, however, it decreased hepatic metastatic invasion of B16M cells inoculated intrasplenically. The study concluded that antimetastatic mechanism of resveratrol was due to the resveratrol induced inhibition of vascular adhesion molecule 1 (VCAM-1) expression in the hepatic sinusoidal endothelium (HSE), which consequently decreased in vitro $\mathrm{B} 16 \mathrm{M}$ cell adhesion to the endothelium via very late activation antigen 4 (VLA-4).

Sale et al. (2004) studied the growth inhibitory properties of putative cancer chemopreventive agent resveratrol against humanderived colon cancer cells HCA-7 and HT-29 cells. As a part of this study, pharmacokinetic properties of 3,4,5,4'-tetramethoxystilbene (DMU 212) were compared with those of resveratrol in the plasma, liver, kidney, lung, heart, brain and small intestinal and colonic mucosa of mice. Both compounds were administered intragastrically at $240 \mathrm{mg} / \mathrm{kg}$ concentration. The results were compared based on the ratios of the area of plasma or tissue concentration vs time curves of resveratrol over DMU $212\left(\mathrm{AUC}_{\mathrm{res}} / \mathrm{AUC}_{\mathrm{DMU} 212}\right)$. The results indicated that the ratio of $\mathrm{AUC}_{\mathrm{res}} / \mathrm{AUC}_{\mathrm{DMU} 212}$ for the plasma, liver, small intestinal and colonic mucosa were $3.5,5$, 0.1 and 0.15 , respectively, thus indicating that resveratrol exhibited significantly higher levels of availability than DMU 212 in the plasma and liver, while DMU 212 exhibited superior availability compared to resveratrol in the small intestine and colon. In addition, the results indicated that resveratrol was metabolised to its sulfate or glucuronate conjugates, while DMU 212 underwent metabolic hydroxylation or single and double O-demethylation. The results from in vitro studies on the human-derived colon cancer cells HCA-7 and HT-29 cells revealed that DMU 212 and resveratrol inhibited the growth of human-derived colon cancer cells with $\mathrm{IC}_{50}$ values of 6 and $26 \mu \mathrm{M}$, thereby indicating that DMU 212 could potentially be used as a chemotherapeutic agent against colon cancer.

Majumdar et al. (2009) studied the potential of using curcumin in synergy with resveratrol to inhibit human colon cancer HCT-116 [p53+/+ or HCT-116 (wt) and HT-29] cells. The results from this study indicated that combination of curcumin with resveratrol was very effective in inhibiting the growth of p53-positive (wt) and p53-negative colon cancer HCT-116 cells in vitro and in vivo in SCID xenografts of colon cancer HCT-116 (wt) cells than either curcumin or resveratrol used alone. The study concluded that the inhibition of tumors in response to curcumin and/or resveratrol was associated with the reduction in proliferation and stimulation of apoptosis supplemented by attenuation of NF- $\mathrm{kB}$ activity. Therefore, combinationed use of curcumin and resveratrol provide a potential preventive/therapeutic strategy for colon cancer.

\subsubsection{Lignans}

Epidemiological studies suggest that there is a plausible link be- 
tween phytoestrogen exposure and a reduced risk of colorectal cancer. Qu et al. (2005) studied the effect of wheat bran from 4 different cultivars in colon cancer SW480 cells. The wheat brans Madison, Ernie and Betty contained 82.9, 52.3 and $42.7 \mu \mathrm{g} / \mathrm{g}$ secoisolariciresinol diglucoside, respectively. In this study, human colon cancer cells SE480 were treated with enterolactone and enterodiol, alone or in combination at $0-40 \mu \mathrm{mol} / \mathrm{L}$ for $24-72 \mathrm{~h}$. It was indicated that treatment of SW480 cells with enterolactone and enterodiol resulted in dose and time-dependent decreases in cell numbers compared to the control samples. The results also indicated that combined effect of enterodiol and enterolactone at 20 and $40 \mu \mathrm{mol} / \mathrm{L}$ had a more severe effect on the cancer cells compared to the individual use of lignan metabolites. The study concluded that cancer cell growth by lignan metabolites seems to be mediated through cytostatic and apoptotic mechanisms.

Felmlee et al. (2009) studied the effect of the flaxseed lignans secoisolariciresinol diglucoside and its aglycones on hyperlipidaemic rats. The results indicated that the lignan component of flaxseed was responsible for the hypochloresterolaemic effects after consumption. Secoisolariciresinol diglucoside and secoisolariciresinol caused dose-dependent reductions in serum and hepatic cholesterol profiles in high cholesterol diet-fed rats.

Feng et al. (2008) studied the effect of metabolites of the lignans enterolactone and enterodiol on osteoblastic differentiation of MG-63 cells. Osteoblasts play a crucial role in the bone formation through proliferation and differentiation. Differentiation of osteoblasts are very important for bone function and confer marked rigidity and strength for bone while maintaining some degree of elasticity. Therefore, it is reported that stimulation of osteoblast differentiation to be an important therapeutic approach for the prevention of bone disease, ie osteoporosis. In this study, osteoblast MG-63 cells were deployed in an in vitro assay system and the evaluated the effects of enterolactone and enterodiol measured by various markers such as mRNA for collagen (col I), ALP, osteocalcin (OC), osteopontin (OP), and osteonectin (ON). The results indicated that both enterolactone and enterodiol have a biphasic effect on the differentiation of osteoblast cells. In addition, both lignan metabolites increased cell viability, alkaline phosphatase (ALP) activity and the transcriptional level of ALP, osteopontin, and osteocalcin in a dose-dependent manner.

\section{Conclusions}

Phenolic compounds undergo various stages of absorption and metabolism, after ingesting them as a part of a food. As a result of various metabolic steps, these metabolites may appear in the plasma as sulfated, glucuronidated or methyl derivatives of the parent phenolic compounds or in some cases, remain unchanged in the plasma or converted to other products. It is important to understand the metabolism of phenolic compounds in the small intestine and fermentation in the colon, because these processes can alter the effects of phenolic compounds on mechanisms of action including free radical scavenging, activation of signaling pathways, and modulation of inflammation response. In this regard, there is a renewed interest to study phenolic compounds and their biological activities, absorption, metabolism and health effects, thus this review will be of interest to the readers in this area. However, due to the diversity of phenolic compounds and colonic microflora, the exact microorganism and the enzymes involved in the catabolism to produce the final catabolite are still largely unknown. Therefore, more thorough research is still needed to fill the existing gaps in this area.

\section{Acknowledgments}

FS thanks the Natural Science and Engineering Research Council (NSERC) of Canada for financial support in the form of a Discovery Grant.

\section{References}

Ackland, M.L., van de Waarsenburg, S., and Jones, R. (2005). Synergistic antiproliferative action of the flavonols quercetin and kaempferol in cultured human cancer cell lines. In Vivo. 19: 69-76.

Akhtar, M.S., and Swamy, M.K. (2018). Anticancer plants: Natural products and biotechnological implements (Volume 2). Springer, Berlin, Germany, pp. 1-564.

Akhtar, S., Meeran, S.M., Katiyar, N., and Katiyar, S.K. (2009). Grape seed proanthocyanidins inhibit the growth of human non-small cell lung cancer xenografts by targeting insulin-like growth factor binding protein-3, tumor cell proliferation, and angiogenic factors. Clin. Cancer Res. 15(3): 821-831.

Akroum, S., Bendjeddou, D., Satta, D., and Lalaoui, K. (2017). Antibacterial, antioxidant and acute toxicity tests on flavonoids extracted from some medicinal plants. Int. J. Green Pharm. 4(3): 165.

Ali, F., Naz, F., Jyoti, S., and Siddique, Y.H. (2017). Health functionality of apigenin: A review. Int. J. Food Prop. 20(6): 1197-1238.

Alshehri, S.M., Shakeel, F., Ibrahim, M.A., Elzayat, E.M., Altamimi, M., Mohsin, K., Almeanazel, O.T., Alkholief, M., Alshetaili, A., Alsulays, B., Alanazi, F.K., and Alsarra, I.A. (2019). Dissolution and bioavailability improvement of bioactive apigenin using solid dispersions prepared by different techniques. Saudi Pharm. J. 27(2): 264-273.

Alvarez-Parrilla, E., Urrea-López, R., and de la Rosa, L.A. (2018). Bioactive components and health effects of pecan nuts and their byproducts: a review. J. Food Bioact. 1(1): 56-92.

Ambigaipalan, P., Oh, W.Y., and Shahidi, F. (2020). Epigallocatechin (EGC) esters as potentialsources of antioxidants. Food Chem. 309: 125609.

Andres-Lacueva, C., Macarulla, M.T., Rotches-Ribalta, M., Boto-Ordóñez, M., Urpi-Sarda, M., Rodríguez, V.M., and Portillo, M.P. (2012). Distribution of resveratrol metabolites in liver, adipose tissue, and skeletal muscle in rats fed different doses of this polyphenol. J. Agric. Food Chem. 60(19): 4833-4840.

Androutsopoulos, V., Wilsher, N., Arroo, R.R.J., and Potter, G.A. (2009). Bioactivation of the phytoestrogen diosmetin by CYP1 cytochromes P450. Cancer Lett. 274(1): 54-60.

Angeloni, C., Spencer, J.P.E., Leoncini, E., Biagi, P.L., and Hrelia, S. (2007). Role of quercetin and its in vivo metabolites in protecting $\mathrm{H} 9 \mathrm{c} 2$ cells against oxidative stress. Biochimie. 89(1): 73-82.

Appeldoorn, M.M., Vincken, J.-P., Aura, A.-M., Hollman, P.C.H., and Gruppen, H. (2009). Procyanidin dimers are metabolized by human microbiota with 2-(3,4-dihydroxyphenyl)acetic acid and 5-(3,4-dihydroxyphenyl)- $\gamma$-valerolactone as the major metabolites. J. Agric. Food Chem. 57(3): 1084-1092.

Asensi, M., Medina, I., Ortega, A., Carretero, J., Baño, M.C., Obrador, E., and Estrela, J.M. (2002). Inhibition of cancer growth by resveratrol is related to its low bioavailability. Free Radic. Biol. Med. 33(3): 387398.

Aura, A.M. (2008). Microbial metabolism of dietary phenolic compounds in the colon. Phytochem. Rev. 7(3): 407-429.

Aura, A.M., Mattila, I., Seppänen-Laakso, T., Miettinen, J., Oksman-Caldentey, K.M., and Orešič, M. (2008). Microbial metabolism of catechin stereoisomers by human faecal microbiota: Comparison of targeted analysis and a non-targeted metabolomics method. Phytochem. Lett. 1(1): 18-22.

Banjerdpongchai, R., Wudtiwai, B., Khaw-on, P., Rachakhom, W., Duangnil, N., and Kongtawelert, P. (2016). Hesperidin from citrus seed induces human hepatocellular carcinoma HepG2 cell apoptosis via both mitochondrial and death receptor pathways. Tumour Biol. 37(1): 227.

Benavente-García, O., Castillo, J., Lorente, J., Ortuño, A., and Del Rio, J.A. (2000). Antioxidant activity of phenolics extracted from Olea europaea L. leaves. Food Chem. 68(4): 457-462. 
Benevides, C.M.D.J., Trindade, B.A., and Lopes, M.V. (2018). Potentialities of legumes in the pharmaceutical industry. J. Anal. Pharm. Res. 7(3): 369-373.

Bhat, T.A., Nambiar, D., Tailor, D., Pal, A., Agarwal, R., and Singh, R.P. (2013). Acacetin inhibits in vitro and in vivo angiogenesis and downregulates Stat signaling and VEGF expression. Cancer Prev. Res. 6(10): 1128-1139.

Blay, M., Espinel, A.E., Delgado, M.A., Baiges, I., Bladé, C., Arola, L., and Salvadó, J. (2010). Isoflavone effect on gene expression profile and biomarkers of inflammation. J. Pharm. Biomed. Anal. 51(2): 382-390.

Bode, L.M., Bunzel, D., Huch, M., Cho, G.-S., Ruhland, D., Bunzel, M., Bub, A., Franz, C.M., and Kulling, S.E. (2013). In vivo and in vitro metabolism of trans-resveratrol by human gut microbiota. Am. J. Clin. Nutr. 97(2): 295-309.

Bolling, B.W., Chen, C.-Y.O., McKay, D.L., and Blumberg, J.B. (2011). Tree nut phytochemicals: composition, antioxidant capacity, bioactivity, impact factors. A systematic review of almonds, Brazils, cashews, hazelnuts, macadamias, pecans, pine nuts, pistachios and walnuts. Nutr. Res. Rev. 24(02): 244-275.

Boué, S.M., Wiese, T.E., Nehls, S., Burow, M.E., Elliott, S., Carter-Wientjes, C.H., Shih, B.Y., McLachlan, J.A., and Cleveland, T.E. (2003). Evaluation of the estrogenic effects of legume extracts containing phytoestrogens. J. Agric. Food Chem. 51: 2193-2199.

Braune, A., and Blaut, M. (2011). Deglycosylation of puerarin and other aromatic C-glucosides by a newly isolated human intestinal bacterium. Environ. Microbiol. 13(2): 482-494.

Braune, A., and Blaut, M. (2016). Bacterial species involved in the conversion of dietary flavonoids in the human gut. Gut Microbes. 7(3): 216-234.

Braune, A., Gütschow, M., Gütschow, G., Engst, W., Blaut, M., and Mikrobiologie, A.G. (2001). Degradation of quercetin and luteolin by Eubacterium ramulus. Appl. Environ. Microbiol. 67(12): 5558-5567.

Budowski, P. (1964). Recent research on sesamin, sesamolin, and related compounds. J. Am. Oil Chem. Soc. 41(4): 280-285.

Carrera, E., Kebir, M.V.O.E., Jacquemond, C., Luro, F., Lozano, Y., and Gaydou, E.M. (2010). Free and bound cinnamic acid derivatives in corsica sweet blond oranges. Nat. Prod. Commun. 5(3): 1934578X1000500.

Castañeda-Ovando, A., Pacheco-Hernández, M.D.L., Páez-Hernández, M.E., Rodríguez, J.A., and Galán-Vidal, C.A. (2009). Chemical studies of anthocyanins: A review. Food Chem. 113(4): 859-871.

Chakraborty, A.K., Funasaka, Y., Komoto, M., and Ichihashi, M. (1998). Effect of arbutin on melanogenic proteins in human melanocytes. Pigment Cell Res. 11(4): 206-212.

Chandrasekara, A., and Shahidi, F. (2010). Content of insoluble bound phenolics in millets and their contribution to antioxidant capacity. J. Agric. Food Chem. 58: 6706-6714.

Chen, A.Y., and Chen, Y.C. (2013). A review of the dietary flavonoid, kaempferol on human health and cancer chemoprevention. Food Chem. 138(4): 2099-2107.

Chen, J.H., and Ho, C.-T. (1997). Antioxidant activities of caffeic acid and its related hydroxycinnamic acid compounds . J. Agric. Food Chem. 45: 2374-2378.

Chien, S.T., Lin, S.S., Wang, C.K., Lee, Y.B., Chen, K.S., Fong, Y., and Shih, Y.W. (2011). Acacetin inhibits the invasion and migration of human non-small cell lung cancer A549 cells by suppressing the p38 $\alpha$ MAPK signaling pathway. Mol. Cell. Biochem. 350(1-2): 135-148.

Chiu, H.-F., Shen, Y.-C., Venkatakrishnan, K., and Wang, C.-K. (2018). Popular functional foods and nutraceuticals with lipid lowering activity and in relation to cardiovascular disease, dyslipidemia, and related complications: An overview. J. Food Bioact. 2(2): 16-27.

Choi, D.Y., Jeong, Y.L., Kim, M.R., Woo, E.R., Yoon, G.K., and Keon, W.K. (2005). Chrysoeriol potently inhibits the induction of nitric oxide synthase by blocking AP-1 activation. J. Biomed. Sci. 12(6): 949-959.

Choi, E.J. (2008). Antioxidative effects of hesperetin against 7,12-dimethylbenz(a)anthracene-induced oxidative stress in mice. Life Sci. 82(21-22): 1059-1064.

Clavel, T., Doré, J., and Blaut, M. (2006). Bioavailability of lignans in human subjects. Nutr. Res. Rev. 19(2): 187-196.

Clifford, M.N. (2000). Miscellaneous phenols in foods and beverages - nature, occurrence and dietary burden. J. Sci. Food Agric. 80(7): 11261137.
Clifford, M.N., and Scalbert, A. (2000). Ellagitannins - nature, occurrence and dietary burden. J. Sci. Food Agric. 80(7): 1118-1125.

Corona, G., Tzounis, X., Assunta Dessì, M., Deiana, M., Debnam, E.S., Visioli, F., and Spencer, J.P.E. (2006). The fate of olive oil polyphenols in the gastrointestinal tract: Implications of gastric and colonic microflora-dependent biotransformation. Free Radic. Res. 40(6): 647-658.

Côrtes, C., Gagnon, N., Benchaar, C., Da Silva, D., Santos, G.T.D., and Petit, H.V. (2008). In vitro metabolism of flax lignans by ruminal and faecal microbiota of dairy cows. J. Appl. Microbiol. 105(5): 1585-1594.

Cremonini, E., Bettaieb, A., Haj, F.G., Fraga, C.G., and Oteiza, P.I. (2016) (-)-Epicatechin improves insulin sensitivity in high fat diet-fed mice. Arch. Biochem. Biophys. 599: 13-21.

Crozier, A., Del Rio, D., and Clifford, M.N. (2010). Bioavailability of dietary flavonoids and phenolic compounds. Mol. Aspects Med. 31(6): 446467.

Dall'Asta, M., Calani, L., Tedeschi, M., Jechiu, L., Brighenti, F., and Del Rio, D. (2012). Identification of microbial metabolites derived from in vitro fecal fermentation of different polyphenolic food sources. Nutrition. 28(2): 197-203.

Daniel, M. (2006). Medicinal plants: Chemistry and properties. CRC press, Boca Raton, Florida, pp. 1-266.

Das, N.P. (1971). Studies on flavonoid metabolism: Absorption and metabolism of (+)-catechin in man. Biochem. Pharmacol. 20(12): 34353445.

Dávalos, A., Castilla, P., Gómez-Cordovés, C., and Bartolomé, B. (2006). Quercetin is bioavailable from a single ingestion of grape juice. Int. J. Food Sci. Nutr. 57(5-6): 391-398.

De Montellano, P.R.O. (2005). Cytochrome P450: Structure, mechanism, and biochemistry. Springer Science \& Business Media, Berlin, Germany, pp. 1-574.

Di Majo, D., Giammanco, M., La Guardia, M., Tripoli, E., Giammanco, S., and Finotti, E. (2005). Flavanones in Citrus fruit: Structure-antioxidant activity relationships. Food Res. Int. 38(10): 1161-1166.

Duarte, J., Pérez-Palencia, R., Vargas, F., Ocete, M.A., Pérez-Vizcaino, F., Zarzuelo, A., and Tamargo, J. (2001). Antihypertensive effects of the flavonoid quercetin in spontaneously hypertensive rats. Br. J. Pharmacol. 133(1): 117-124.

El Gharras, H. (2009). Polyphenols: Food sources, properties and applications - a review. Int. J. Food Sci. Technol. 44(12): 2512-2518.

Engler, M.B., Engler, M.M., Chen, C.Y., Malloy, M.J., Browne, A., Chiu, E.Y., Kwak, H.-K., Milbury, P., Paul, S.M., Blumberg, J., and Mietus-Snyder, M.L. (2004). Flavonoid-rich dark chocolate improves endothelial function and increases plasma epicatechin concentrations in healthy adults. J. Am. Coll. Nutr. 23(3): 197-204.

Erlejman, A.G., Verstraeten, S.V., Fraga, C.G., and Oteiza, P.I. (2004). The interaction of flavonoids with membranes: Potential determinant of flavonoid antioxidant effects. Free Radic. Res. 38(12): 1311-1320.

Espín, J.C., González-Barrio, R., Cerdá, B., López-Bote, C., Rey, A.I., and Tomás-Barberán, F.A. (2007). Iberian pig as a model to clarify obscure points in the bioavailability and metabolism of ellagitannins in humans. J. Agric. Food Chem. 55(25): 10476-10485.

Felgines, C., Texier, O., Morand, C., Manach, C., Scalbert, A., Régerat, F., and Rémésy, C. (2000). Bioavailability of the flavanone naringenin and its glycosides in rats. Am. J. Physiol. Gastrointest. Liver Physiol. 279(6): G1148-G1154.

Felmlee, M.A., Woo, G., Simko, E., Krol, E.S., Muir, A.D., and Alcorn, J. (2009). Effects of the flaxseed lignans secoisolariciresinol diglucoside and its aglycone on serum and hepatic lipids in hyperlipidaemic rats. Br. J. Nutr. 102(3): 361-369.

Feng, J., Shi, Z., and Ye, Z. (2008). Effects of metabolites of the lignans enterolactone and enterodiol on osteoblastic differentiation of MG-63 cells. Biol. Pharm. Bull. 31(6): 1067-1070.

Formica, J.V., and Regelson, W. (1995). Review of the biology of quercetin and related bioflavonoids. Food Chem. Toxicol. 33: 1061-1080.

Frankenfeld, C.L. (2012). O-desmethylangolensin: The importance of equol's lesser known cousin to human health. Adv. Nutr. 2(4): 317-324.

Funayama, M., Arakawa, H., Yamamoto, R., Nishino, T., Shin, T., and Mu rao, S. (1995). Effects of $\alpha$ - and $\beta$-arbutin on activity of tyrosinases from mushroom and mouse melanoma. Biosci. Biotechnol. Biochem. 59(1): 143-144.

Gaya, P., Medina, M., Sánchez-Jiménez, A., and Landete, J.M. (2016). Phy- 
toestrogen metabolism by adult human gut microbiota. Molecules. 21(8): 1034

Gonthier, M.P., Cheynier, V., Donovan, J.L., Manach, C., Morand, C., Mila, I., Lapierre, C., Rémésy, C., and Scalbert, A. (2018). Microbial aromatic acid metabolites formed in the gut account for a major fraction of the polyphenols excreted in urine of rats fed red wine polyphenols. J. Nutr. 133(2): 461-467.

González-Barrio, R., Borges, G., Mullen, W., and Crozier, A. (2010). Bioavailability of anthocyanins and ellagitannins following consumption of raspberries by healthy humans and subjects with an ileostomy. J. Agric. Food Chem. 58(7): 3933-3939.

Gossé, F., Guyot, S., Roussi, S., Lobstein, A., Fischer, B., Seiler, N., and Raul, F. (2005). Chemopreventive properties of apple procyanidins on human colon cancer-derived metastatic SW620 cells and in a rat model of colon carcinogenesis. Carcinogenesis. 26(7): 1291-1295.

Graf, B.A., Milbury, P.E., and Blumberg, J.B. (2005). Flavonols, flavones, flavanones, and human health: Epidemiological evidence. J. Med. Food. 8(3): 281-290

Grasel, F.D.S., Ferrão, M.F., and Wolf, C.R. (2016). Development of methodology for identification the nature of the polyphenolic extracts by FTIR associated with multivariate analysis. Spectrochim. Acta - Part A Mol. Biomol. Spectrosc 153: 94-101.

Gu, L., Kelm, M.A., Hammerstone, J.F., Beecher, G., Holden, J., Haytowitz, D., and Prior, R.L. (2003). Screening of foods containing proanthocyanidins and their structural characterization using LC-MS/MS and thiolytic degradation. J. Agric. Food Chem. 51(25): 7513-7521.

Guengerich, F.P. (2007). Cytochrome p450 and chemical toxicology. Chem. Res. Toxicol. 21(1): 70-83.

Gupta, S., Hastak, K., Ahmad, N., Lewin, J.S., and Mukhtar, H. (2001). Inhibition of prostate carcinogenesis in TRAMP mice by oral infusion of green tea polyphenols. Proc. Natl. Acad. Sci. 98(18): 10350-10355.

Gutierrez-Salmean, G., Ciaraldi, T.P., Nogueira, L., Barboza, J., Taub, P.R., Hogan, M.C., Henry, R.R., Meaney, E., Villarreal, F., Ceballos, G., and Ramirez-Sanchez, I. (2014). Effects of (-)-epicatechin on molecular modulators of skeletal muscle growth and differentiation. J. Nutr. Biochem. 25(1): 91-94.

Haidari, F., Keshavarz, S.A., Rashidi, M.R., and Shahi, M.M. (2009). Orange juice and hesperetin supplementation to hyperuricemic rats alter oxidative stress markers and xanthine oxidoreductase activity. J. Clin. Biochem. Nutr. 45(3): 285-291.

Hajialyani, M., Hosein Farzaei, M., Echeverría, J., Nabavi, S.M., Uriarte, E., and Sobarzo-Sánchez, E. (2019). Hesperidin as a Neuroprotective Agent: A Review of Animal and Clinical Evidence. Molecules 24(3): 648.

Häkkinen, S.H., Kärenlampi, S.O., Heinonen, I.M., Mykkänen, H.M., and Törronen, A.R. (1999). Content of the flavonols quercetin, myricetin, and kaempferol in 25 edible berries. J. Agric. Food Chem. 47(6): 2274-2279.

Hardman, W.E. (2014). Diet components can suppress inflammation and reduce cancer risk. Nutr. Res. Pract. 8(3): 233-240.

Hatcher, D.W., and Kruger, J.E. (1997). Simple phenolic acids in flours prepared from canadian wheat: relationship to ash content, color, and polyphenol oxidase activity. Cereal Chem. J. 74(3): 337-343.

Hedlund, T.E., Johannes, W.U., and Miller, G.J. (2003). Soy isoflavonoid equol modulates the growth of benign and malignant prostatic epithelial cells in vitro. Prostate. 54(1): 68-78.

Heleno, S.A., Martins, A., Queiroz, M.J.R.P., and Ferreira, I.C.F.R. (2015) Bioactivity of phenolic acids: Metabolites versus parent compounds: A review. Food Chem. 173: 501-513.

Heo, H.J., Kim, D.O., Shin, S.C., Kim, M.J., Kim, B.G., and Shin, D.H. (2004) Effect of antioxidant flavanone, naringenin, from Citrus junos on neuroprotection. J. Agric. Food Chem. 52(6): 1520-1525.

Herath, W., Iqrar Khan, S., and Ahmad Khan, I. (2013). Microbial metabolism. Part 14. Isolation and bioactivity evaluation of microbial metabolites of resveratrol. Nat. Prod. Res. 27(16): 1437-1444.

Hernández-Aquino, E., and Muriel, P. (2018). Beneficial effects of naringenin in liver diseases: Molecular mechanisms. World J. Gastroenterol. 24(16): 1679-1707.

Hostetler, G.L., Ralston, R.A., and Schwartz, S.J. (2017). Flavones: Food sources, bioavailability, metabolism, and bioactivity. Am. Soc. Nutr. 8: 423-435.
Hsu, Y.L., Kuo, P.L., Liu, C.F., and Lin, C.C. (2004). Acacetin-induced cell cycle arrest and apoptosis in human non-small cell lung cancer A549 cells. Cancer Lett. 212(1): 53-60.

Ikemura, M., Sasaki, Y., Giddings, J.C., and Yamamoto, J. (2012). Preventive effects of hesperidin, glucosyl hesperidin and naringin on hypertension and cerebral thrombosis in stroke-prone spontaneously hypertensive rats. Phyther. Res. 26(9): 1272-1277.

Issenberg, P., Kornerich, M.R., and Lustre, A.O. (1971). Recovery of phenolic wood smoke components from smoked foods and model systems. J. Food Sci. 36(1): 107-109.

Jackman, K.A., Woodman, O.L., Chrissobolis, S., and Sobey, C.G. (2007) Vasorelaxant and antioxidant activity of the isoflavone metabolite equol in carotid and cerebral arteries. Brain Res. 1141: 99-107.

Jaramillo, S., Lopez, S., Varela, L.M., Rodriguez-Arcos, R., Jimenez, A., Abia, R., Guillen, R., and Muriana, F.J.G. (2010). The flavonol isorhamnetin exhibits cytotoxic effects on human colon cancer cells. J. Agric. Food Chem. 58(20): 10869-10875.

Jeng, K., and Hou, R. (2005). Sesamin and sesamolin: Natures therapeutic lignans. Curr. Enzym. Inhib. 1(1): 11-20.

Ju, J., Hong, J., Zhou, J., Pan, Z., Bose, M., Liao, J., Yang, G., Liu, Y.Y., Hou, Z., Lin, Y., Ma, J., Shih, W.J., Carothers, A.M., and Yang, C.S. (2005) Inhibition of intestinal tumorigenesis in Apc min/+ mice by (-)-epigallocatechin-3-gallate, the major catechin in green tea. Cancer Res 65(22): 10623-10631.

Kang, M.H., Naito, M., Tsujihara, N., and Osawa, T. (1998). Sesamolin inhibits lipid peroxidation in rat liver and kidney. J. Nutr. 128(6): 10181022

Kaur, M., Velmurugan, B., Rajamanickam, S., Agarwal, R., and Agarwal, C. (2009). Gallic acid, an active constituent of grape seed extract, exhibits anti-proliferative, pro-apoptotic and anti-tumorigenic effects against prostate carcinoma xenograft growth in nude mice. Pharm. Res. 26(9): 2133-2140.

Kemperman, R.A., Bolca, S., Roger, L.C., and Vaughan, E.E. (2010). Nove approaches for analysing gut microbes and dietary polyphenols: Challenges and opportunities. Microbiology. 156(11): 3224-3231.

Khan, A., and Gilani, A.H. (2006). Selective bronchodilatory effect of rooibos tea (Aspalathus linearis) and its flavonoid, chrysoeriol. Eur. J. Nutr. 45(8): 463-469.

Khoo, H.E., Azlan, A., Tang, S.T., and Lim, S.M. (2017). Anthocyanidins and anthocyanins: Colored pigments as food, pharmaceutical in gredients, and the potential health benefits. Food Nutr. Res. 61(1): 1361779 .

Kim, M.J., Kwak, H.S., and Kim, S.S. (2018). Effects of germination on protein, $\mathrm{Y}$-aminobutyric acid, phenolic acids, and antioxidant capacity in wheat. Molecules. 23(9): 2244.

Kim, Y.H., Lee, Y.S., and Choi, E.M. (2010). Chrysoeriol isolated from Eurya cilliata leaves protects MC3T3-E1 cells against hydrogen peroxideinduced inhibition of osteoblastic differentiation. J. Appl. Toxicol. 30(7): 666-673.

Kleemann, R., Verschuren, L., Morrison, M., Zadelaar, S, van Erk, M.J. Wielinga, P.Y., and Kooistra, T. (2011). Anti-inflammatory, anti-proliferative and anti-atherosclerotic effects of quercetin in human in vitro and in vivo models. Atherosclerosis 218(1): 44-52.

Konishi, Y., Hitomi, Y., and Yoshioka, E. (2004). Intestinal absorption of $p$ coumaric and gallic acids in rats after oral administration. J. Agric. Food Chem. 52(9): 2527-2532.

Konishi, Y., Kobayashi, S., and Shimizu, M. (2003). Transepithelial transport of $p$-coumaric acid and gallic acid in caco-2 cell monolayers. Biosci. Biotechnol. Biochem. 67(11): 2317-2324.

Konishi, Y., Zhao, Z., and Shimizu, M. (2006). Phenolic acids are absorbed from the rat stomach with different absorption rates. J. Agric. Food Chem. 54(20): 7539-7543.

Koutsos, A., Tuohy, K.M., and Lovegrove, J.A. (2015). Apples and cardiovas cular health -is the gut microbiota a core consideration? Nutrients. 7(6): 3959-3998.

Kubo, I., Masuoka, N., Ha, T.J., and Tsujimoto, K. (2006). Antioxidant activ ity of anacardic acids. Food Chem. 99(3): 555-562.

Kumano, T., Fujiki, E., Hashimoto, Y., and Kobayashi, M. (2016). Discovery of a sesamin-metabolizing microorganism and a new enzyme. Proc. Natl. Acad. Sci. U.S.A. 113(32): 9087-9092.

Kwik-Uribe, C., and Bektash, R.M. (2008). Cocoa flavanols - measurement, 
bioavailability and bioactivity. Asia Pac. J. Clin. Nutr. 17(Suppl 1): 280-283.

La, V.D., Tanabe, S., and Grenier, D. (2009). Naringenin inhibits human osteoclastogenesis and osteoclastic bone resorption. J. Periodontal Res. 44(2): 193-198.

Lafay, S., and Gil-Izquierdo, A. (2008). Bioavailability of phenolic acids. Phytochem. Rev. 7(2): 301-311.

Lee, J.S., Kim, H.J., and Lee, Y.S. (2003). A new anti-HIV flavonoid glucuronide from Chrysanthemum morifolium. Planta Med. 69(9): 859-861.

Lee, K.A., Lee, S.H., Lee, Y.J., Baeg, S.M., and Shim, J.H. (2012). Hesperidin induces apoptosis by inhibiting Sp1 and its regulatory protein in MSTO-211H cells. Biomol. Ther. 20(3): 273-279.

Lee, P.G., Kim, J., Kim, E.-J., Lee, S.-H., Choi, K.-Y., Kazlauskas, R.J., and Kim, B.-G. (2017). Biosynthesis of (-)-5-hydroxy-equol and 5-hydroxy-dehydroequol from soy isoflavone, genistein using microbial whole cell bioconversion. ACS Chem. Biol. 12(11): 2883-2890.

Li, S., Gosslau, A., Lange, K., and Ho, C.-T. (2019). Profiled tea extracts exemplifying the importance of characterizing food bioactives: Opinion piece. J. Food Bioact. 5: 1-5.

Licciardello, F., Arena, E., Rizzo, V., and Fallico, B. (2018). Contribution of Blood Orange-Based Beverages to Bioactive Compounds Intake. Front. Chem. 6: 374.

Lin, Y., Shi, R., Wang, X., and Shen, H.-M. (2008). Luteolin, a flavonoid with potentials for cancer prevention and therapy. Curr Cancer Drug Targets. 8(7): 634-646.

Lin, Y., Wu, B., Li, Z., Hong, T., Chen, M., Tan, Y., Jiang, J., and Huang, C. (2012). Metabolite identification of myricetin in rats using HPLC coupled with ESI-MS. Chromatographia. 75(11-12): 655-660.

Liu, L.Z., Fang, J., Zhou, Q., Hu, X., Shi, X., and Jiang, B.-H. (2005). Apigenin inhibits expression of vascular endothelial growth factor and angiogenesis in human lung cancer cells: Implication of chemoprevention of lung cancer. Mol. Pharmacol. 68(3): 635-643.

Liu, L.Z., Jing, Y., Jiang, L.L., Jiang, X.E., Jiang, Y., Rojanasakul, Y., and Jiang, B.H. (2011). Acacetin inhibits VEGF expression, tumor angiogenesis and growth through AKT/HIF-1 $\alpha$ pathway. Biochem. Biophys. Res. Commun. 413(2): 299-305.

Lu, G., Liao, J., Yang, G., Reuhl, K.R., Hao, X., and Yang, C.S. (2006a). Inhibition of adenoma progression to adenocarcinoma in a 4-(methylnitrosamino)-1-(3-pyridyl)-1-butanone-induced lung tumorigenesis model in $\mathrm{A} / \mathrm{J}$ mice by tea polyphenols and caffeine. Cancer Res. 66(23): 11494-11501.

Lu, H.F., Chie, Y.J., Yang, M.S., Lu, K.W., Fu, J.J., Yang, J.S., Chen, H.Y., Hsia, T.C., Ma, C.Y., Ip, S.W., and Chung, J.G. (2011). Apigenin induces apoptosis in human lung cancer $\mathrm{H} 460$ cells through caspase- and mitochondria-dependent pathways. Hum. Exp. Toxicol. 30(8): 1053-1061.

Lu, Z., Nie, G., Belton, P.S., Tang, H., and Zhao, B. (2006b). Structure-activity relationship analysis of antioxidant ability and neuroprotective effect of gallic acid derivatives. Neurochem. Int. 48(4): 263-274.

Lund, T.D., Blake, C., Bu, L., Hamaker, A.N., and Lephart, E.D. (2011). Equol an isoflavonoid: potential for improved prostate health, in vitro and in vivo evidence. Reprod. Biol. Endocrinol. 9(1): 4.

Maeda, K., and Fukuda, M. (1996). Arbutin: Mechanism of its depigmenting action in human melanocyte culture. J. Pharmacol. Exp. Ther. 276(2): 765-769.

Majumdar, A.P.N., Banerjee, S., Nautiyal, J., Patel, B.B., Patel, V., Du, J., Yu, Y., Elliott, A.A., Levi, E., and Sarkar, F.H. (2009). Curcumin synergizes with resveratrol to inhibit colon cancer. Nutr. Cancer. 61(4): 544-553.

Manach, C., Scalbert, A., Morand, C., Rémésy, C., and Jiménez, L. (2004). Polyphenols: Food sources and bioavailability. Am. J. Clin. Nutr. 79(5): 727-747.

Mateos, R., Goya, L., and Bravo, L. (2005). Metabolism of the olive oil phenols hydroxytyrosol, tyrosol, and hydroxytyrosyl acetate by human hepatoma HepG2 cells. J. Agric. Food Chem. 53(26): 9897-9905.

Mathey, J., Mardon, J., Fokialakis, N., Puel, C., Kati-Coulibaly, S., Mitakou, S., Bennetau-Pelissero, C., Lamothe, V., Davicco, M.J., Lebecque, P., Horcajada, M.N., and Coxam, V. (2007). Modulation of soy isoflavones bioavailability and subsequent effects on bone health in ovariectomized rats: The case for equol. Osteoporos. Int. 18(5): 671-679.

Matos, M.J., Santana, L., Uriarte, E., Abreu, O.A., Molina, E., and Yordi, E.G. (2015). Coumarins-an important class of phytochemicals. Phytochemicals - Isolation, Characterisation and Role in Human Health.
In: Rao, V., and Rao, L. (Ed.). InTechOpen, London, UK, pp. 113-140.

Mei, Y.Z., Liu, R.X., Wang, D.P., Wang, X., and Dai, C.C. (2015). Biocatalysis and biotransformation of resveratrol in microorganisms. Biotechnol. Lett. 37(1): 9-18.

Mertens-Talcott, S.U., Jilma-Stohlawetz, P., Rios, J., Hingorani, L., and Derendorf, H. (2006). Absorption, metabolism, and antioxidant effects of pomegranate (Punica granatum L.) polyphenols after ingestion of a standardized extract in healthy human volunteers. J. Agric. Food Chem. 54(23): 8956-8961.

Milenkovic, D., Deval, C., Dubray, C., Mazur, A., and Morand, C. (2011). Hesperidin displays relevant role in the nutrigenomic effect of orange juice on blood leukocytes in human volunteers: A randomized controlled cross-over study. PLoS One. 6(11): e26669.

Mirfat, A.H.S., Amin, I., Kartinee, K.N., Muhajir, H., and Shukri, M.A.M (2018). Underutilised fruits: A review of phytochemistry and biological properties. J. Food Bioact. 1(1): 2-30.

Mishra, B., Priyadarsini, K.I., Kumar, M.S., Unnikrishnan, M.K., and Mohan, H. (2003). Effect of O-glycosilation on the antioxidant activity and free radical reactions of a plant flavonoid, chrysoeriol. Bioorg. Med. Chem. 11(13): 2677-2685.

Miyashita, K., and Hosokawa, M. (2018). Health impact of marine carotenoids. J. Food Bioact. 1(1): 31-40.

Monagas, M., Urpi-Sarda, M., Sánchez-Patán, F., Llorach, R., Garrido, I., Gómez-Cordovés, C., Andres-Lacueva, C., and Bartolomé, B. (2010). Insights into the metabolism and microbial biotransformation of dietary flavan-3-ols and the bioactivity of their metabolites. Food Funct. 1(3): 233.

Monks, T.J., Hanzlik, R.P., Cohen, G.M., Ross, D., and Graham, D.G. (1992). Quinone chemistry and toxicity. Toxicol. Appl. Pharmacol. 112(1): 2-16.

Morales, P., and Haza, A.I. (2012). Selective apoptotic effects of piceatannol and myricetin in human cancer cells. J. Appl. Toxicol. 32(12): 986-993.

Mosele, J.I., Martín-Peláez, S., Macià, A., Farràs, M., Valls, R.M., Catalán, Ú., and Motilva, M.J. (2014). Faecal microbial metabolism of olive oil phenolic compounds: In vitro and in vivo approaches. Mol. Nutr. Food Res. 58(9): 1809-1819.

Muhammad, D.R.A., and Dewettinck, K. (2017). Cinnamon and its derivatives as potential ingredient in functional food-A review. Int. J. Food Prop. 20(28): 2237-2263

Mukker, J.K., Michel, D., Muir, A.D., Krol, E.S., and Alcorn, J. (2014). Permeability and conjugative metabolism of flaxseed lignans by caco-2 human intestinal cells. J. Nat. Prod. 77(1): 29-34.

Mullen, W., Rouanet, J.-M., Auger, C., Teissèdre, P.-L., Caldwell, S.T., Hartley, R.C., Lean, M.E.J., Edwards, C.A., and Crozier, A. (2008). Bioavailability of [2-14 C]Quercetin-4'-glucoside in rats. J. Agric. Food Chem. 56(24): 12127-12137.

Mullin, W.J., and Emery, J.P.H. (1992). Determination of alkylresorcinols in cereal-based foods. J. Agric. Food Chem. 40(11): 2127-2130.

Naczk, M., and Shahidi, F. (2006). Phenolics in cereals, fruits and vegetables: Occurrence, extraction and analysis, J. Pharmaceut. Biomed. Anal. 41: 1523-1542.

Nagao, T., Komine, Y., Soga, S., Meguro, S., Hase, T., Tanaka, Y., and Tokimitsu, I. (2005). Ingestion of a tea rich in catechins leads to a reduction in body fat and malondialdehyde-modified LDL in men. Am. J. Clin. Nutr. 81(1): 122-129.

Narayana, K.R., Reddy, M.S., Chaluvadi, M.R., and Krishna, D.R. (2001). Bioflavonoids classification, pharmacological, biochemical effects and therapeutic potential. Indian J. Pharmacol. 33: 2-16.

Neilson, A.P., Goodrich, K.M., and Ferruzzi, M.G. (2017). Bioavailability and metabolism of bioactive compounds from foods. Nutrition in the prevention and treatment of disease. In: Coulston, A.M., Boushey, C.J., Ferruzzi, M.G., and Delahanty, L.M. (Ed.). Academic Press, Cambridge, MA, pp. 301-319

Niemeyer, H.B., Honig, D.M., Kulling, S.E., and Metzler, M. (2003). Studies on the metabolism of the plant lignans secoisolariciresinol and matairesinol. J. Agric. Food Chem. 51(21): 6317-6325.

Oh, W.Y., and Shahidi, F. (2017). Lipophilization of resveratrol and effects on antioxidant activities. J. Agric. Food Chem. 65: 8617-8625.

Oh, W.Y., and Shahidi, F. (2018). Antioxidant activity of resveratrol ester derivatives in food and biological model systems. Food Chem. 261: 
267-273.

Oh, W.Y., Ambigaipalan, P., and Shahidi, F. (2019). Preparation of quercetin esters and their antioxidant activity. J. Agric. Food Chem. 67: 1065310659.

Oh, W.Y., Chiou, Y.-S., Pan, M.-H., and Shahidi, F. (2019). Lipophilised resveratrol affects the generation of reactive nitrogen species in murine macrophages and cell viability of human cancer cell lines. J. Food Bioact. 7: 73-77.

Orrego-Lagarón, N., Martínez-Huélamo, M., Vallverdú-Queralt, A., Lamuela-Raventos, R.M., and Escribano-Ferrer, E. (2015). High gastrointestinal permeability and local metabolism of naringenin: Influence of antibiotic treatment on absorption and metabolism. Br. J. Nutr. 114(02): 169-180

Orrego-Lagarón, N., Vallverdú-Queralt, A., Martínez-Huélamo, M., Lamuela-Raventos, R.M., and Escribano-Ferrer, E. (2016). Metabolic profile of naringenin in the stomach and colon using liquid chromatography/ electrospray ionization linear ion trap quadrupole-orbitrap-mass spectrometry (LC-ESI-LTQ-Orbitrap-MS) and LC-ESI-MS/MS. J. Pharm. Biomed. Anal. 120: 38-45.

Ou, K., and Gu, L. (2014). Absorption and metabolism of proanthocyanidins. J. Funct. Foods. 7: 43-53.

Palacz-Wróbel, M. (2018). Health-promoting properties of apigenin and kaempferol. Ann. Pharmacol. Pharm. 3(2): 1-5.

Panche, A.N., Diwan, A.D., and Chandra, S.R. (2016). Flavonoids: An overview. J. Nutr. Sci. 5: 1-15.

Parhiz, H., Roohbakhsh, A., Soltani, F., Rezaee, R., and Iranshahi, M. (2015). Antioxidant and anti-inflammatory properties of the citrus flavonoids hesperidin and hesperetin: An updated review of their molecular mechanisms and experimental models. Phyther. Res. 29(3): 323-331.

Pari, L., and Shagirtha, K. (2012). Hesperetin protects against oxidative stress related hepatic dysfunction by cadmium in rats. Exp. Toxicol. Pathol. 64(5): 513-520.

Patel, K., Gadewar, M., Tahilyani, V., and Patel, D.K. (2013). A review on pharmacological and analytical aspects of diosmetin: A concise report. Chin. J. Integr. Med. 19(10): 792-800

Pereira-Caro, G., Ludwig, I.A., Polyviou, T., Malkova, D., García, A., Moreno-Rojas, J.M., and Crozier, A. (2016). Identification of plasma and urinary metabolites and catabolites derived from orange juice (poly) phenols: Analysis by high-performance liquid chromatography-highresolution mass spectrometry. J. Agric. Food Chem. 64(28): 57245735.

Perera, N., Ambigaipalan, P., and Shahidi, F. (2018). Epigallocatechin gallate (EGCG) esters with different chain lengths fatty acids and their antioxidant activity in food and biological systems. J. Food Bioact. 1(1): 124-133.

Perez-Vizcaino, F., and Duarte, J. (2010). Flavonols and cardiovascular disease. Mol. Aspects Med. 31(6): 478-494

Pietta, P., Minoggio, M., and Bramati, L. (2003). Plant polyphenols: Structure, occurrence and bioactivity. Stud. Nat. Prod. Chem. 28: 257-312.

Piwowarski, J.P., Granica, S., Stefańska, J., and Kiss, A.K. (2016). Differences in metabolism of ellagitannins by human gut microbiota ex vivo cultures. J. Nat. Prod. 79(12): 3022-3030.

Prasad, R., Vaid, M., and Katiyar, S.K. (2012). Grape proanthocyanidin inhibit pancreatic cancer cell growth in vitro and in vivo through induction of apoptosis and by targeting the PI3K/Akt pathway. PLoS One 7(8): e43064.

Qu, H., Madl, R.L., Takemoto, D.J., Baybutt, R.C., and Wang, W. (2005). Lignans are involved in the antitumor activity of wheat bran in colon cancer SW480 cells. J. Nutr. 135(3): 598-602.

Rawel, H.M., Czajka, D., Rohn, S., and Kroll, J. (2002). Interactions of different phenolic acids and flavonoids with soy proteins. Int. J. Biol. Macromol. 30: 137-150.

Rechner, A.R., Smith, M.A., Kuhnle, G., Gibson, G.R., Debnam, E.S., Srai, S.K.S., Moore, K.P., and Rice-Evans, C.A. (2004). Colonic metabolism of dietary polyphenols: Influence of structure on microbial fermentation products. Free Radic. Biol. Med. 36(2): 212-225

Rechner, A.R., Spencer, J., Kuhnle, G., Hahn, U., and Rice-Evans, C. (2001). Novel biomarkers of the metabolism of caffeic acid derivatives in vivo. Free Radic. Biol. Med. 30(11): 1213-1222.

Rimbach, G., De Pascual-Teresa, S., Ewins, B.A., Matsugo, S., Uchida, Y., Minihane, A.M., Turner, R., Vafeiadou, K., and Weinberg, P.D. (2003)
Antioxidant and free radical scavenging activity of isoflavone metabolites. Xenobiotica. 33(9): 913-925.

Rio, D.D., Borges, G., and Crozier, A. (2010). Berry flavonoids and phenolics: Bioavailability and evidence of protective effects. Br. J. Nutr. 104: S67-S90.

Roowi, S., Stalmach, A., Mullen, W., Lean, M.E.J., Edwards, C.A., and Crozier, A. (2010). Green tea flavan-3-ols: Colonic degradation and urinary excretion of catabolites by humans. J. Agric. Food Chem. 58(2): 1296-1304.

Rossi, M., Negri, E., Parpinel, M., Lagiou, P., Bosetti, C., Talamini, R., Montella, M., Giacosa, A., Franceschi, S., and La Vecchia, C. (2010). Proanthocyanidins and the risk of colorectal cancer in Italy. Cancer Causes Control 21(2): 243-250

Russo, R., Chandradhara, D., and de Tommasi, N. (2018). Comparative bioavailability of two diosmin formulations after oral administration to healthy volunteers. Molecules. 23(9): 2174.

Sale, S., Verschoyle, R.D., Boocock, D., Jones, D.J.L., Wilsher, N., Ruparelia, K.C., Potter, G.A., Farmer, P.B., Steward, W.P., and Gescher, A.J. (2004). Pharmacokinetics in mice and growth-inhibitory properties of the putative cancer chemopreventive agent resveratrol and the synthetic analogue trans 3,4,5,4'-tetramethoxystilbene. Br. J. Cancer. 90(3): 736-744.

Sameermahmood, Z., Raji, L., Saravanan, T., Vaidya, A., Mohan, V., and Balasubramanyam, M. (2010). Gallic acid protects RINm5F $\beta$-cells from glucolipotoxicity by its antiapoptotic and insulin-secretagogue actions. Phyther. Res. 24(S1): S83-S94.

Sánchez-Patán, F., Chioua, M., Garrido, I., Cueva, C., Samadi, A., MarcoContelles, J., Moreno-Arribas, M.V., Bartolomé, B., and Monagas, M. (2011). Synthesis, analytical features, and biological relevance of 5-(3',4'-Dihydroxyphenyl)- $\psi$-valerolactone, a microbial metabolite derived from the catabolism of dietary flavan-3-ols. J. Agric. Food Chem. 59(13): 7083-7091.

Scalbert, A., and Williamson, G. (2000). Chocolate: Modern science investigates an ancient medicine. J. Med. Food. 3(2): 121-125.

Schroeter, H., Heiss, C., Balzer, J., Kleinbongard, P., Keen, C.L., Hollenberg, N.K., Sies, H., Kwik-Uribe, C., Schmitz, H.H., and Kelm, M. (2006). (-)-Epicatechin mediates beneficial effects of flavanol-rich cocoa on vascular function in humans. Proc. Natl. Acad. Sci. 103(4): 1024-1029.

Seelinger, G., Merfort, I., and Schempp, C. (2008a). Anti-oxidant, antiinflammatory and anti-allergic activities of luteolin. Planta Med. 74(14): 1667-1677.

Seelinger, G., Merfort, I., Wölfle, U., and Schempp, C.M. (2008b). Anticarcinogenic effects of the flavonoid luteolin. Molecules. 13(10): 2628-2651.

Seeram, N.P., Lee, R., and Heber, D. (2004). Bioavailability of ellagic acid in human plasma after consumption of ellagitannins from pomegranate (Punica granatum L.) juice. Clin. Chim. Acta. 348(1-2): 63-68.

Senanayake, S.N. (2018). Rosemary extract as a natural source of bioactive compounds. J. Food Bioact. 2(2): 51-57.

Serra, A., Macl, A., Romero, M.P., Anglés, N., Morelló, J.R., and Motilva M.J. (2011). Metabolic pathways of the colonic metabolism of procyanidins (monomers and dimers) and alkaloids. Food Chem. 126(3): 1127-1137.

Serra, A., Macià, A., Romero, M.-P., Reguant, J., Ortega, N., and Motilva M.-J. (2012). Metabolic pathways of the colonic metabolism of flavonoids (flavonols, flavones and flavanones) and phenolic acids. Food Chem. 130(2): 383-393.

Setchell, K.D., Zhao, X., Jha, P., Heubi, J.E., and Brown, N.M. (2009). The pharmacokinetic behavior of the soy isoflavone metabolite S-(-)eq$\mathrm{uol}$ and its diastereoisomer R-(+)equol in healthy adults determined by using stable-isotope-labeled tracers. Am. J. Clin. Nutr. 90(4) 1029-1037.

Setchell, K.D.R., and Cassidy, A. (2018). Dietary isoflavones: Biological effects and relevance to human health. J. Nutr. 129(3): 758S-767S

Shahidi, F., and Ambigaipalan, P. (2015). Phenolics and polyphenolics in foods, beverages and spices: Antioxidant activity and health effects A review. J. Funct. Foods 18: 820-897.

Shahidi, F., and Naczk, M. (2003). Phenolics in food and nutraceuticals. CRC press, Boca Raton, Florida, pp. 1-558.

Shahidi, F., and Peng, H. (2018). Bioaccessibility and bioavailability of phenolic compounds. J. Food Bioact. 4: 11-68. 
Shahidi, F., and Yeo, J.D. (2016). Insoluble-bound phenolics in food. Molecules. 21(9): 1216

Shahidi, F., and Yeo, J.D. (2018). Bioactivities of phenolics by focusing on suppression of chronic diseases: A review. Int. J. Mol. Sci. 19: 1573.

Shahidi, F.V., Vamadevan, V., Oh, W.Y., and Peng, H. (2019). Phenolic compounds in agri-food by-products, their bioavailability and health effects. J. Food Bioact. 5: 57-119.

Shahrzad, S., Aoyagi, K., Winter, A., Koyama, A., and Bitsch, I. (2001). Pharmacokinetics of gallic acid and its relative bioavailability from tea in healthy humans. J. Nutrs. 131: 1207-1210.

Shimoi, K., Suzuki, M., Goda, T., Furugori, M., Hara, Y., Yamamoto, H., Kinae, N., Okada, H., and Takase, S. (2002). Intestinal absorption of luteolin and luteolin 7-O-6-glucoside in rats and humans. FEBS Lett. 438(3): 220-224.

Shin, D.W., Kim, S.N., Lee, S.M., Lee, W., Song, M.J., Park, S.M., Lee, T.R., Baik, J.-H., Kim, H.K., Hong, J.-H., and Noh, M. (2009). (-)-Catechin promotes adipocyte differentiation in human bone marrow mesenchymal stem cells through PPARY transactivation. Biochem. Pharmacol. 77(1): 125-133.

Shor, D., Sathyapalan, T., Atkin, S.L., and Thatcher, N.J. (2012). Does equol production determine soy endocrine effects? Eur. J. Nutr. 51(4): 389-398.

Shukla, S., and Gupta, S. (2010). Apigenin: A promising molecule for cancer prevention. Pharm. Res. 27(6): 962-978.

Singh, R.P., Agrawal, P., Yim, D., Agarwal, C., and Agarwal, R. (2005). Acacetin inhibits cell growth and cell cycle progression, and induces apoptosis in human prostate cancer cells: structure-activity relationship with linarin and linarin acetate. Carcinogenesis. 26(4): 845-854.

Smith, G.E., and Griffiths, L.A. (1970). Metabolism of myricitrin and 3,4,5-trihydroxyphenylacetic acid. Biochem. J. 118(3): 53P-54P.

Spadafranca, A., Martinez Conesa, C., Sirini, S., and Testolin, G. (2010). Effect of dark chocolate on plasma epicatechin levels, DNA resistance to oxidative stress and total antioxidant activity in healthy subjects. Br. J. Nutr. 103(07): 1008

Spencer, J.P.E., Schroeter, H., Rechner, A.R., and Rice-Evans, C. (2001). Bioavailability of flavan-3-ols and procyanidins: Gastrointestinal tract influences and their relevance to bioactive forms in vivo. Antioxid. Redox Signal. 3(6): 1023-1039.

Springer, M., and Moco, S. (2019). Resveratrol and its human metabolites-Effects on metabolic health and obesity. Nutrients. 11(1): 143.

Stevens, J.F., and Maier, C.S. (2016). The chemistry of gut microbial metabolism of polyphenols. Phytochem. Rev. 15(3): 425-444.

Stoupi, S., Drynan, J.W., Barron, D., and Clifford, M.N. (2010). Procyanidin B2 catabolism by human fecal microflora: Partial characterization of 'dimeric' intermediates. Arch. Biochem. Biophys. 501(1): 73-78.

Subash, S., and Subramanian, A.P. (2009). Morin a flavonoid exerts antioxidant potential in chronic hyperammonemic rats: A biochemical and histopathological study. Mol. Cell Biochem. 327: 153-161.

Sun, W., Frost, B., and Liu, J. (2017). Oleuropein, unexpected benefits!. Oncotarget 8: 17409-17409.

Sun, Y., Zhou, D., and Shahidi, F. (2018). Antioxidant properties of tyrosol and hydroxytyrosol saturated fatty acid esters. Food Chem. 245: $1262-1268$

Tang, R., Chen, K., Cosentino, M., and Lee, K.-H. (1994). Apigenin-7-O- $\beta-$ D-glucopyranoside, an anti-HIV principle from Kummerowia striata. Bioorg. Med. Chem. Lett. 4(3): 455-458.

Tomás-Barberán, F.A., and Clifford, M.N. (2000a). Dietary hydroxybenzoic acid derivatives - nature, occurrence and dietary burden. J. Sci. Food Agric. 80(7): 1024-1032.

Tomás-Barberán, F.A., and Clifford, M.N. (2000b). Flavanones, chalcones and dihydrochalcones - nature, occurrence and dietary burden. J. Sci. Food Agric. 80(7): 1073-1080.

Tomás-Barberán, F.A., García-Villalba, R., González-Sarrías, A., Selma, M.V., and Espín, J.C. (2014). Ellagic acid metabolism by human gut microbiota: Consistent observation of three urolithin phenotypes in intervention trials, independent of food source, age, and health status. J. Agric. Food Chem. 62(28): 6535-6538.

Tsao, R. (2010). Chemistry and biochemistry of dietary polyphenols. Nutrients. 2(12): 1231-1246.

Tulipani, S., Huelamo, M.M., Ribalta, M.R., Estruch, R., Ferrer, E.E., AndresLacueva, C., Illan, M., and Lamuela-Raventós, R.M. (2012). Oil matrix effects on plasma exposure and urinary excretion of phenolic compounds from tomato sauces: Evidence from a human pilot study. Food Chem. 130: 581-590.

Valdez, J.C., and Bolling, B.W. (2019). Anthocyanins and intestinal barrier function: A review. J.Food Bioactives 5: 18-30.

Viskupičová, J., Ondrejovič, M., and Šturdík, E. (2008). Absorption, bioavailability, and metabolism of flavonoids. J. Food Nutr. Res. 47(4): 151-162.

Vissiennon, C., Nieber, K., Kelber, O., and Butterweck, V. (2012). Route of administration determines the anxiolytic activity of the flavonols kaempferol, quercetin and myricetin - are they prodrugs? J. Nutr. Biochem. 23(7): 733-740.

Wan, M.L., Ling, K.H., El-Nezami, H., and Wang, M. (2018). Oxyresveratrol protective effects against deoxynivalenol-induced intestinal barrier dysfunction and bacterial translocation on porcine intestinal epithelial IPEC-J2 cells. J. Food Bioact. 1(1): 116-123.

Wang, H., Chambers, E., and Kan, J. (2018). Sensory characteristics of combinations of phenolic compounds potentially associated with smoked aroma in foods. Molecules. 23(8): 1867.

Wang, J., Yang, Z., Lin, L., Zhao, Z., Liu, Z., and Liu, X. (2012). Protective effect of naringenin against lead-induced oxidative stress in rats. Biol. Trace Elem. Res. 146(3): 354-359.

Wang, L., Tu, Y.C., Lian, T.W., Hung, J.T., Yen, J.H., and Wu, M.J. (2006). Distinctive antioxidant and antiinflammatory effects of flavonols. J. Agric. Food Chem. 54(26): 9798-9804.

Wilmsen, P.K., Spada, D.S., and Salvador, M. (2005). Antioxidant activity of the flavonoid hesperidin in chemical and biological systems. J. Agric. Food Chem. 53(12): 4757-4761.

Xu, C., Li, C.Y.-T., and Kong, A.-N.T. (2005). Induction of phase I, II and III drug metabolism/transport by xenobiotics. Arch. Pharm. Res. 28(3) 249-268.

Xu, H., Kulkarni, K.H., Singh, R., Yang, Z., Wang, S.W.J., Tam, V.H., and Hu, M. (2009). Disposition of naringenin via glucuronidation pathway is affected by compensating efflux transporters of hydrophilic glucuronides. Mol. Pharm. 6: 1703-1715.

Xu, Y., Xin, Y., Diao, Y., Lu, C., Fu, J., Luo, L., and Yin, Z. (2011). Synergistic effects of apigenin and paclitaxel on apoptosis of cancer cells. PLoS One. 6(12): e29169.

Yamamoto, M., Jokura, H., Suzuki, A., Hase, T., and Shimotoyodome, A. (2013). Effects of continuous ingestion of hesperidin and glucosyl hesperidin on vascular gene expression in spontaneously hypertensive rats. J. Nutr. Sci. Vitaminol. (Tokyo) 59(5): 470-473.

Yan, X., Qi, M., Li, P., Zhan, Y., and Shao, H. (2017). Apigenin in cancer therapy: Anti-cancer effects and mechanisms of action. Cell Biosci. 7(1): 50 .

Yeo, J.D., and Shahidi, F. (2017). Effect of hydrothermal processing on changes of insoluble-bound phenolics of lentils. J. Funct. Foods 38 : 716-722.

Yeo, J.D., and Shahidi, F. (2019). Analysis of flavonoid-protein interactions by advanced techniques. Encyclopedia of Food Chemistry. In: Varelis, P., Melton, L., and Shahidi, F. (Ed.). Elsevier Science \& Technology, pp. 539-543.

Yim, S.-H., Kim, H.J., and Lee, I.-S. (2003). A polyacetylene and flavonoids from Cirsium rhinoceros. Arch. Pharm. Res. 26(2): 128-131.

Yoshiara, L.Y., Mandarino, J.M.G., Carrão-Panizzi, M.C., Madeira, T.B., da Silva, J.B., de Camargo, A.C., Shahidi, F., and Ida, E.I. (2018). Germination changes the isoflavone profile and increases the antioxidant potential of soybean. J. Food Bioact. 3: 144-150.

Yu, C., Shin, Y.G., Chow, A., Li, Y., Kosmeder, J.W., Lee, Y.S., Hirschelman, W.H., Pezzuto, J.M., Mehta, R.G., and van Breemen, R.B. (2002). Human, rat, and mouse metabolism of resveratrol. Pharm. Res. 19(12): 1907-1914.

Yu, L., and Beta, T. (2015). Identification and antioxidant properties of phenolic compounds during production of bread from purple wheat grains. Molecules. 20(9): 15525-15549.

Zhang, J., Liu, D., Huang, Y., Gao, Y., and Qian, S. (2012). Biopharmaceutics classification and intestinal absorption study of apigenin. Int. J. Pharm. 436(1-2): 311-317.

Zhao, G., Han, X., Cheng, W., Ni, J., Zhang, Y., Lin, J., and Song, Z. (2017). Apigenin inhibits proliferation and invasion, and induces apoptosis and cell cycle arrest in human melanoma cells. Oncol. Rep. 37(4): 
$2277-2285$.

Zhao, J., Dasmahapatra, A.K., Khan, S.I., and Khan, I.A. (2008). Anti-aromatase activity of the constituents from damiana (Turnera diffusa). J. Ethnopharmacol. 120(3): 387-393.

Zhong, Y., Ma, C.-M., and Shahidi, F. (2012). Antioxidant and antiviral activities of lipophilic epigallocatechin gallate (EGCG) derivatives. J. Funct. Foods 4: 87-93.

Zhu, Y., Wu, J., Li, S., Wang, X., Liang, Z., Xu, X., Xu, X., Hu, Z., Lin, Y., Chen, H., Qin, J., Mao, Q., and Xie, L. (2015). Apigenin inhibits migration and invasion via modulation of epithelial mesenchymal transition in prostate cancer. Mol. Med. Rep. 11(2): 1004-1008.

Zieliñska, M., Kostrzewa, A., Ignatowicz, E., and Budzianowski, J. (2001). The flavonoids, quercetin and isorhamnetin 3-O-acylglucosides diminish neutrophil oxidative metabolism and lipid peroxidation. Acta Biochim. Pol. 48(1): 183-189.

Zong, L., Inoue, M., Nose, M., Kojima, K., Sakaguchi, N., Isuzugawa, K., Takeda, T., and Ogihara, Y. (1999). Metabolic fate of gallic acid orally administered to rats. Biol. Pharm. Bull. 22(3): 326-329. 